\title{
SECOND MOMENT OF THE CENTRAL VALUES OF THE SYMMETRIC SQUARE L-FUNCTIONS
}

\author{
DISSERTATION \\ Presented in Partial Fulfillment of the Requirements for the Degree Doctor of \\ Philosophy in the Graduate School of the Ohio State University
}

By

Wing Chung Jonathan Lam,

Graduate Program in Mathematics

The Ohio State University

2015

\author{
Dissertation Committee: \\ Professor Wenzhi Luo, Advisor \\ Professor James Cogdell \\ Professor Roman Holowinsky
}


(c) Copyright by

Wing Chung Jonathan Lam

2015 


\begin{abstract}
In this thesis we obtain the sharp upper bound for the moments of $L$-functions of conductor $K^{2+\epsilon}$ in short interval at the center of the critical strip. This includes the case of the second moment of symmetric square $L$-function associated to holomorphic cusp forms of weight $k$, over the interval $K \leq k \leq K+K^{\frac{1}{3}}$, and the first moment of Rankin-Selberg $L$-function of symmetric square lift of holomorphic cusp forms of weight $k$ and a fixed Maass cusp form over the interval $K \leq k \leq K+K^{\frac{1}{3}}$.
\end{abstract}


To my mother 


\section{ACKNOWLEDGMENTS}

First and foremost I thank my advisor, Professor Wenzhi Luo, who teaches me the analytic theory of automorphic forms and is always generous with his time, encouragements and ideas. I am forever grateful for his belief in me at the time when I felt less certain about myself. Without his helps and patience, this thesis would have been impossible.

I would also like to thank Professor James Cogdell and Professor Holowinsky for agreeing to serve in the thesis committee.

Most of what I know about the algebraic theory of Auotmorphic Representation for reductive groups, I learnt it through participating in the Automorphic Forms Seminar. I would like to express my gratitude to the members of the seminar, especially Professor James Cogdell and the late Professor Cary Rader.

Thanks are due to Rudy, Chelsea, Little Gatsby and Noah, who are the first friends

I made in this country and remain among the most loyal ones. I would also like to thank my climbing partner Chris for introducing me to the sport of rock climbing and mountaineering, and for all the (mis)adventures and near-death experiences.

Last but not least, I thank my mother for all her patience and love. For being the beginning of this adventure, for all the suffering through the difficult years. 


\begin{abstract}
VITA
$1987 \ldots \ldots \ldots \ldots \ldots \ldots \ldots \ldots \ldots \ldots \ldots \ldots \ldots \ldots \ldots \ldots$ Born in Hong Kong

$2009 \ldots \ldots \ldots \ldots \ldots \ldots \ldots \ldots \ldots \ldots \ldots \ldots \ldots \ldots \ldots$ B.Sc. in Mathematics, The Chinese University of Hong Kong

2009-Present .................. Graduate Teaching Associate, The Ohio State University
\end{abstract}

\title{
PUBLICATIONS
}

J.W.C. Lam, A local large sieve inequality for cusp forms, vol 26, Fascicule 3, Journal de Théorie des numbers de Bordeaux

J.W.C. Lam, Second moment of the central values of the symmetric square Lfunctions, published online on August 1 2014, the Ramanujan Journal

\section{FIELDS OF STUDY}

Major Field: Mathematics

Specialization: Analytic Number Theory 


\section{TABLE OF CONTENTS}

Abstract ................................. ii

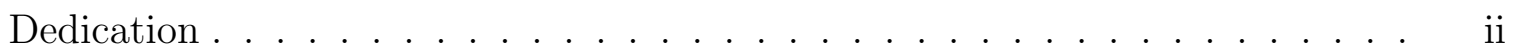

Acknowledgments . . . . . . . . . . . . . . . . . . iv

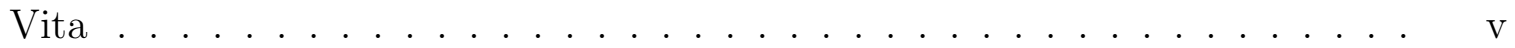

CHAPTER PAGE

$1 \quad$ Introduction . . . . . . . . . . . . . . . . . . . 1

1.1 Definitions and Notations . . . . . . . . . . . . . . . . 1

1.1.1 Holomorphic cusp forms . . . . . . . . . . . . . . 1

1.1.2 Maass forms and Eisenstein series . . . . . . . . . . . 4

1.1.3 Hecke Theory . . . . . . . . . . . . . . . . . . 6

1.1.4 Automorphic $L$-functions . . . . . . . . . . . . . . . . . . . 7

1.2 Main Results . . . . . . . . . . . . . . . . . . . . 10

1.2.1 Local large sieve inequalities for $G L(2)$ cusp forms . . . . . 10

1.2.2 Mean value of symmetric square $L$-functions . . . . . . . . 13

1.2.3 First moment of Rankin-Selberg $L$-functions. . . . . . . . . 13

2 Large sieve inequalities . . . . . . . . . . . . . . . . 15

2.1 Holomorphic cusp forms of integral weight $k$. . . . . . . . . . 15

2.1.1 First manipulations . . . . . . . . . . . . . . 16

2.1.2 The diagonal contribution . . . . . . . . . . . . . . . . 17

2.1.3 The off-diagonal contribution . . . . . . . . . . . . 17

2.1.4 Proof of Lemma 2.1.2 . . . . . . . . . . . . . . . . . . 19

2.2 Holomorphic cusp forms of half integral weight . . . . . . . . . . 21

2.2.1 First manipulation . . . . . . . . . . . . . . . . 21

2.2.2 The diagonal contribution . . . . . . . . . . . . . . 22

2.2.3 The off-diagonal contribution . . . . . . . . . . . 22

2.3 Maass cusp forms . . . . . . . . . . . . . . . . . . . . . 34

2.3.1 First Manipulation ... . . . . . . . . . . . . 35

2.3.2 The diagonal contribution . . . . . . . . . . . 36 
2.3.3 The off-diagonal contribution . . . . . . . . . 36

3 The Second moment of the central values of the symmetric square $L$ functions . . . . . . . . . . . . . . . 40

3.1 Shortening the length of summation . . . . . . . . . . . . 41

3.2 Further manipulation . . . . . . . . . . . . . . . . 45

3.3 The off-diagonal contribution . . . . . . . . . . . . 47

3.3.1 Restricting the sum over $c \ldots \ldots \ldots$. . . . . . . . . 47

3.3.2 Applying the stationary phase lemma . . . . . . . . . . . 49

3.3.3 Applying the Poisson summation formula . . . . . . . . 51

3.3.4 Application of the two dimensional Van der Corput lemma 53

4 The first moment of the central values of the Rankin-Selberg $L$-functions 56

4.1 First manipulation . . . . . . . . . . . . . . . . 58

4.2 Further reduction to the sum over $c \ldots \ldots \ldots$. . . . . . . 61

4.3 Applying the Stationary Phase Lemma and the Hecke relation . . 64

4.4 Evaluation of the Sums of the Kloosterman sum . . . . . . . . 68

4.5 Applying Voronoi formula . . . . . . . . . . . . . . . 72

4.6 Reduction of the range of sum over $n \ldots \ldots \ldots$

4.7 Bounding the Oscillatory integral . . . . . . . . . . . . 77

4.8 Estimating the sum of twisted exponential sums . . . . . . 80

4.9 Proof of Lemma $4.4 .1 \ldots \ldots \ldots$

4.10 Concluding the proof of the main theorem . . . . . . . . 88

Bibliography . . . . . . . . . . . . . . . . . . . 92 


\section{CHAPTER 1 INTRODUCTION}

Men wanted for hazardous journey. Low wages, bitter cold, long hours of complete darkness. Safe return doubtful. Honour and recognition in event of success

\subsection{Definitions and Notations}

In this section we will introduce basic concepts and definitions in the theory of automorphic forms including results that we will use throughout this thesis.

\subsubsection{Holomorphic cusp forms}

Let $\mathbb{H}$ be the upper half plane, $S L_{2}(\mathbb{Z})$ be the modular group, i.e. group of $2 \times 2$ matrices with integral entries and determinant 1 . For each integer $q$, one can define the Hecke congruence subgroup of level $q$, denoted $\Gamma_{0}(q)$, by

$$
\Gamma_{0}(q)=\left\{\left(\begin{array}{ll}
a & b \\
c & d
\end{array}\right) \in S L_{2}(\mathbb{Z}): c \equiv 0(q)\right\}
$$

and the stabilizing group of a group $\Gamma$ by

$$
\Gamma_{x}=\{\gamma \in \Gamma: \gamma x=x\}
$$


The modular group (hence the Hecke congruence subgroups) acts on the upper half plane as follows: for each $z \in \mathbb{H}, g \in\left(\begin{array}{cc}a & b \\ c & d\end{array}\right) \in S L_{2}(\mathbb{Z})$, we have

$$
g \cdot z:=\frac{a z+b}{c z+d}
$$

The cusps of $\Gamma_{0}(q)$ are rational numbers and $\infty$. Let $f: \mathbb{H} \rightarrow \mathbb{C}$ be a function on the upper half plane. We define for each $k$

$$
\begin{gathered}
\left(\left.f\right|_{k} g\right)(z):=j(g, z)^{-k} f(g \cdot z) \\
j(g, z):=c z+d \text { for } g=\left(\begin{array}{cc}
a & b \\
c & d
\end{array}\right) .
\end{gathered}
$$

A holomorphic modular form of weight $k$, level $q$, and trivial nebentypus (which we assume from now on and will not mention again in this thesis) is a holomorphic function $f$ on $\mathbb{H}$, such that

$$
\left.f\right|_{k} \gamma=f \text { for all } \gamma \in \Gamma_{0}(q)
$$

and is holomorphic at all cusps of $\Gamma_{0}(q)$.

By holomorphic at a cusp, we mean the following: Let $\mathfrak{a}$ be a cusp of $\Gamma_{0}(q)$, then there exists $\sigma_{\mathfrak{a}} \in S L_{2}(\mathbb{R})$ such that $\sigma_{\mathfrak{a}} \infty=\mathfrak{a}, \sigma_{\mathfrak{a}}^{-1} \gamma_{\mathfrak{a}} \sigma_{\mathfrak{a}}=\left(\begin{array}{ll}1 & 1 \\ 0 & 1\end{array}\right)$ where $\gamma_{\mathfrak{a}}$ is the generator of $\Gamma_{0}(q)_{\mathfrak{a}}$. Let $f_{\mathfrak{a}}:=\left.f\right|_{k} \sigma_{\mathfrak{a}}$. We have the Laurent series expansion

$$
f_{\mathfrak{a}}(z)=\sum_{n \geq n_{\mathfrak{a}}} \hat{f}_{\mathfrak{a}}(n) e(n z)
$$

(where $e(z)=\exp (2 \pi i z)$ ) for some integer $n_{\mathfrak{a}}$, and $\hat{f}_{\mathfrak{a}}\left(n_{\mathfrak{a}}\right) \neq 0$. We say that $f$ is holomorphic at $\mathfrak{a}$ if it is meromorphic and $n_{\mathfrak{a}} \geq 0$. If moreover, we have $n_{\mathfrak{a}}>0$, we say that $f$ vanishes at $\mathfrak{a}$. If $f$ vanishes at all cusps, $f$ is said to be a cusp form. 
In the case when $k$ is half integral, we have to be slightly more careful, as it involves taking fractional root of a complex numbers. In this case, everything is the same as before, except we define

$$
\begin{aligned}
j(\gamma, z) & :=\theta(\gamma \cdot z) / \theta(z) \text { for } \gamma \in \Gamma_{0}(4) \\
\text { where } \quad \theta(z) & :=\sum_{-\infty}^{\infty} e\left(n^{2} z\right) .
\end{aligned}
$$

The set of holomorphic cusp forms of weight $k$ (integral or half-integral) and level $q$ (in the case of half-integral weight forms, we require that $4 \mid q$ ) forms a finite dimensional vector space, which is denoted by $S_{k}\left(\Gamma_{0}(q)\right) . S_{k}\left(\Gamma_{0}(q)\right)$ has an inner product called the Petersson inner product defined as follows: For $f, g \in S_{k}\left(\Gamma_{0}(q)\right)$.

$$
<f, g>:=\frac{1}{\operatorname{vol}\left(X_{0}(q)\right)} \int_{X_{0}(q)} y^{k} f(z) \overline{g(z)} \frac{d x d y}{y^{2}}
$$

where $X_{0}(q)=\Gamma_{0}(q) \backslash \mathbb{H}$.

For each $f \in S_{k}\left(\Gamma_{0}(q)\right), f$ has a Fourier expansion $f(z)=\sum_{n \geq 1} a_{f}(n) n^{\frac{k-1}{2}} e(n z)$. Define $\rho_{f}(n)$ as

$$
\rho_{f}(n)=\sqrt{\frac{(k-1) !}{(4 \pi)^{k-1}}} a_{f}(n) .
$$

Take any orthonormal basis $B_{k, q}$ of $S_{k}\left(\Gamma_{0}(q)\right)$, we have the following Petersson trace formulae:

Theorem 1.1.1. For integer $k>1$. For any positive integers $m, n$,

$$
\begin{gathered}
\frac{1}{\operatorname{vol}\left(X_{0}(q)\right)} \sum_{f \in B_{k, q}} \rho_{f}(m) \overline{\rho_{f}(n)}=(k-1) \delta_{m n} \\
+2 \pi i^{-k}(k-1) \sum_{c \equiv 0(q)} \frac{S(m, n ; c)}{c} J_{k-1}\left(\frac{4 \pi \sqrt{m n}}{c}\right)
\end{gathered}
$$

where

$$
S(m, n ; c)=\sum_{\substack{d(c) \\(d, c)=1}} e\left(\frac{d m+\bar{d} n}{c}\right)
$$

is the Kloosterman sum, $J_{k-1}(x)$ is the $J$-Bessel function. 
Theorem 1.1.2. For half integral $k>0$. For any $m, n$,

$$
\begin{array}{r}
\frac{1}{\operatorname{vol}\left(X_{0}(q)\right)} \sum_{f \in B_{k, q}} \rho_{f}(m) \overline{\rho_{f}(n)}=(k-1) \delta_{m n} \\
+2 \pi i^{-k} \sum_{c \equiv 0(M)} \frac{K_{k}(m, n ; c)}{c} J_{k-1}\left(\frac{4 \pi \sqrt{m n}}{c}\right)
\end{array}
$$

where $K_{k}(m, n ; c)$ is the generalized Kloosterman sum,

$$
K_{k}(m, n ; c)=\sum_{d(c)} \epsilon^{-2 k}\left(\frac{c}{d}\right) e\left(\frac{m \bar{d}+n d}{c}\right)
$$

with $d \bar{d} \equiv 1(c), \epsilon_{d}=\left\{\begin{array}{l}1 \text { if } d \equiv 1(4) \\ i \text { if } d \equiv-1(4)\end{array} \quad\right.$, and $\left(\frac{c}{d}\right)$ is the extended Kronecker symbol (see P.388 of [8]).

\subsubsection{Maass forms and Eisenstein series}

Let $\Delta=-y^{2}\left(\frac{\partial^{2}}{\partial x^{2}}+\frac{\partial^{2}}{\partial y^{2}}\right)$ be the hyperbolic Laplace operator. A Maass form of level $q$ is a function $f \in L^{2}\left(\Gamma_{0}(q) \backslash \mathbb{H}\right)$ such that

$$
\Delta f=s(1-s) f \text { for some } s \in \mathbb{C}
$$

and

$$
\left.f\right|_{\gamma}=f \text { for all } \gamma \in \Gamma_{0}(q)
$$

Similar to the holomorphic, one can define cusipdal Maass forms (see P.6). Each Maass forms $f$ has a Fourier expansion (at the cusp $\infty$ )

$$
f(z)=a y^{s}+b y^{1-s}+\sqrt{y} \sum_{n \neq 0} a_{f}(n) K_{s-\frac{1}{2}}(2 \pi|n| y) e(n x)
$$

where $\Delta f=s(1-s) f$ and $K_{s}(y)$ is the $K$-Bessel function. Similar to holomorphic cusp forms, one can define an inner product as follows: For two Maass forms $f, g$ of level $q$,

$$
<f, g>:=\frac{1}{\operatorname{vol}\left(X_{0}(q)\right)} \int_{X_{0}(q)} f(z) \overline{g(z)} \frac{d x d y}{y^{2}}
$$


For each cusp $\mathfrak{a}$ of $\Gamma_{0}(q)$, we can define the Eisenstein series

$$
E_{\mathfrak{a}, q}(z, s)=\sum_{\gamma \in \Gamma_{\mathfrak{a}} \backslash \Gamma}\left(\operatorname{Im} \sigma_{\mathfrak{a}}^{-1} \gamma z\right)^{s}
$$

where $\Gamma=\Gamma_{0}(q), \Gamma_{\infty}=\left\{\gamma \in S L_{2}(\mathbb{Z}): \gamma \infty=\infty\right\}$, and $\Gamma_{\mathfrak{a}}=\sigma_{\mathfrak{a}} \Gamma_{\infty} \sigma_{\mathfrak{a}}^{-1}$. At each cusp $\mathfrak{b}$, it has the Fourier expansion

$$
E_{\mathfrak{a}, q}\left(\sigma_{\mathfrak{b}} z, s\right)=\delta_{\mathfrak{a}, \mathfrak{b}} y^{s}+\phi_{\mathfrak{a}, \mathfrak{b}, q} y^{1-s}+\sqrt{y} \sum_{n \neq 0} \phi_{\mathfrak{a} \cdot \mathfrak{b}, q}(n, s) K_{s-\frac{1}{2}}(2 \pi|n| y) e(n x) .
$$

The analogue of the Petersson trace formula in this case is the Kuznetsov trace formula. Let $\left(u_{j}\right)$ be an orthonormal basis of Maass forms for $\Gamma_{0}(q)$ with $q \geq 1$ and let $\lambda_{j}=s_{j}\left(1-s_{j}\right)$ with $s_{j}=\frac{1}{2}+i t_{j}$ be the corresponding eigenvalues of the Laplace operator. Let

$$
u_{j}=a y^{s_{j}}+b y^{1-s_{j}}+\sqrt{y} \sum_{n \neq 0} \rho_{j}(n) K_{i t_{j}}(2 \pi|n| y) e(n x)
$$

be the Fourier expansion of $u_{j}$ at the cusp $\infty$. Let $\mathfrak{a}$ be a cusp of $\Gamma_{0}(q)$, and $\tau_{\mathfrak{a}, q}(n, r)=$ $\phi_{\mathfrak{a}, \infty, q}\left(n, \frac{1}{2}+i r\right)$. Then for a function $h: \mathbb{C} \rightarrow \mathbb{C}$ such that

$$
\left\{\begin{array}{l}
h(t)=h(-t), \\
\text { is holomorphic in }|\operatorname{Im}(t)| \leq \frac{1}{2}+\delta, \\
\text { and } h(t) \ll(|t|+1)^{-2-\delta}
\end{array}\right.
$$

for some $\delta>0$. We have

Theorem 1.1.3. (Kuznetsov trace formula) for any $m, n>0$,

$$
\begin{aligned}
& \sum_{j=1}^{\infty} \overline{\rho_{j}(m)} \rho_{j}(n) \frac{h\left(t_{j}\right)}{\cosh \pi t_{j}}+\sum_{\mathfrak{a}} \frac{1}{4 \pi} \int_{-\infty}^{\infty} \overline{\tau_{\mathfrak{a}, q}(m, r)} \tau_{\mathfrak{a}, q}(n, r) \frac{h(r) d r}{\cosh \pi r} \\
= & \delta_{m n} g_{0}+\sum_{c \equiv 0(q)} \frac{S(m, n ; c)}{c} g\left(\frac{4 \pi \sqrt{m n}}{c}\right)
\end{aligned}
$$


where

$$
g_{0}=\frac{1}{\pi^{2}} \int_{-\infty}^{\infty} r h(r) \tanh \pi t d r
$$

and

$$
g(x)=\frac{2 i}{\pi} \int_{-\infty}^{\infty} J_{2 i r}(x) \frac{r h(r) d r}{\cosh \pi r}
$$

\subsubsection{Hecke Theory}

Fix integers $k \geq 1, q \geq 1, n \geq 1$. One defines

$$
\left(T_{n} f\right)(z):=\frac{1}{n} \sum_{a d=n} a^{k} \sum_{0 \leq b<d} f\left(\frac{a z+b}{d}\right) .
$$

It is well-known that for each $(n, q)=1, T_{n}$ sends holomorphic cusp forms of weight $k$ and level $q$ to holomorphic cusp forms of weight $k$ and level $q$.

In the Maass form case, the Hecke operator is defined differently and will still be denoted by $T_{n}$,

$$
\left(T_{n} f\right)(z):=\frac{1}{\sqrt{n}} \sum_{a d=n} \sum_{0 \leq b<d} f\left(\frac{a z+b}{d}\right) .
$$

It also sends Maass cusp forms of level $q$ to Maass cusp forms of level $q$. Of crucial importance is the following well-known Theorem.

Theorem 1.1.4. There is an orthonormal basis of the space of cusp forms (holomorphic or Maass) of level $q$ which consists of eigenfunctions of all the Hecke operators $T_{n}$ for $(n, q)=1$.

A ( $L^{2}$-normalized $)$ holomorphic cusp form or Maass cusp form of level $q$ who is the common eigenfunction of all $T_{n}$ with $(n, q)=1$ is called a Hecke eigenform and for a Hecke eigenform $f$ such that $T_{n} f=\lambda_{n} f$. We have in the holomorphic case

$$
\lambda_{n} a_{f}(1)=a_{f}(n) \text { if }(n, q)=1
$$


where $f(z)=\sum_{n \geq 1} a_{f}(n) n^{(k-1) / 2} e(n z)$, in this case

$$
\left|a_{f}(1)\right|^{2}=\frac{(4 \pi)^{k-1}}{(k-1) !} \frac{2 \pi^{2}}{q L\left(1, \operatorname{sym}^{2} f\right)} \operatorname{vol}\left(X_{0}(q)\right) ;
$$

and in the Maass form case

$$
\lambda_{n} \nu(1)=\nu(n) \text { if }(n, q)=1
$$

where

$$
\nu(n)=\sqrt{\frac{|n| q}{\cosh \pi t_{j}}} \rho(n)
$$

and

$$
f(z)=\sqrt{y} \sum_{n \neq 0} \rho(n) K_{i t_{j}}(2 \pi|n| y) e(n x)
$$

with $t_{j} \in \mathbb{R}$ or $\frac{1}{2}<s_{j}<1$.

By Theorem 2.3.1, for each integral $k, q$, we can find an orthonormal basis consisting of Hecke eigenforms (called a Hecke basis). Similarly in the Maass form case.

Take any $q^{\prime}$ and $d$ with $d q^{\prime} \mid q$. If $f$ is a holomorphic cusp form of weight $k$ and level $q^{\prime}$ then $f_{d}(z):=f(d z)$ is a holomorphic cusp form weight $k$ and level $q$. A primitive holomorphic cusp form (or holomorphic new cusp form) is a holomorphic cusp form $f$ such that $f$ can not be written as $g_{d}$ for any cusp form $g$ of lower level.

\subsubsection{Automorphic $L$-functions}

Let $f$ be a primitive holomorphic cusp forms (i.e. not induced from a lower level) of weight $k$ and level $q$. Let

$$
f(z)=\sum_{n \geq 1} \lambda_{f}(n) n^{\frac{k-1}{2}} e(n z)
$$

be its Fourier expansion at the $\operatorname{cusp} \infty$, with $\lambda_{f}(n)$ being its $n$-th Hecke eigenvalue. Then

$$
L(f, s)=\sum_{n \geq 1} \lambda_{f}(n) n^{-s}=\prod_{p}\left(1-\lambda_{f}(p) p^{-s}+p^{-2 s}\right)^{-1}
$$


is an $L$-function of degree 2 with conductor $q$ and gamma factor given by

$$
\gamma(f, s)=2^{\frac{3-k}{2}} \sqrt{\pi}(2 \pi)^{-s} \Gamma\left(s+\frac{k-1}{2}\right) .
$$

Then the analytic conductors are

$$
\begin{aligned}
& q(f, s) \leq q(|s|+|k|+3)^{2}, \\
& q(f) \asymp q k^{2} .
\end{aligned}
$$

Similarly, let $\phi$ be a primitive Maass cusp form of level $q$ which is an egienfunction of the Laplace operator with eigenvalue $\lambda=\frac{1}{4}+r^{2}$, where $r \in \mathbb{R}$ or ir $\in\left[0, \frac{1}{2}\right]$. Writing its Fourier expansion at $\infty$ in the form

$$
\phi(z)=\sqrt{y} \sum_{n \neq 0} \rho(n) K_{i r}(\pi|n| y) e(n x)
$$

we associate with $\phi$ the $L$-function

$$
L(\phi, s)=\sum_{n \geq 1} \rho(n) n^{-s}=\prod_{p}\left(1-\rho(p) p^{-s}+p^{-2 s}\right)^{-1}
$$

of conductor $q$, with gamma factor

$$
\gamma(\phi, s)=\pi^{-s} \Gamma\left(\frac{s+\delta+i r}{2}\right) \Gamma\left(\frac{s+\delta-i r}{2}\right)
$$

where $\delta=0$ if $\phi$ is even (i.e. $\rho(n)=\rho(-n)$ ) and $\delta=1$ otherwise. Then the analytic conductors are

$$
\begin{aligned}
& q(\phi, s) \leq q(|s|+|r|+3)^{2} \\
& q(\phi)=q(|r|+3)^{2} \asymp \lambda q .
\end{aligned}
$$

The next $L$-function we are going to introduce is the symmetric square $L$-function associated to holomorphic cusp forms. It is proved in 1976 by S. Gelbart and H. Jacquet that the symmetric square lift of a $G L(2)$ automorphic form is a $G L(3)$ automorphic form, this is one of the first validation of the functoriality conjecture of 
R. Langlands. The symmetric square $L$-function is the $L$-function associates to this $G L(3)$ automorphic form. The symmetric square $L$-function associated to holomorphic cusp forms is defined as follows: Let $k$ be an even positive number, $f$ be a holomorphic Hecke cusp form of weight $k$, level 1 and of $L^{2}$-norm 1 . The symmetric square $L$-function associated to $f$ is defined to be

$$
L\left(s, \operatorname{sym}^{2} f\right)=\zeta(2 s) \sum_{n=1}^{\infty} \frac{\lambda_{f}\left(n^{2}\right)}{n^{s}}
$$

where $\lambda_{f}(m)$ is the $m$-th Hecke eigenvalue. $L\left(s, \operatorname{sym}^{2} f\right)$ analytically extends to an entire function on $\mathbb{C}$ and satisfies the functional equation

$$
L_{\infty}(s) L\left(s, \operatorname{sym}^{2} f\right)=L_{\infty}(1-s) L\left(1-s, \operatorname{sym}^{2} f\right)
$$

where

$$
\begin{aligned}
L_{\infty}(s) & =\pi^{-\frac{3}{2} s} \Gamma\left(\frac{s+1}{2}\right) \Gamma\left(\frac{s+k-1}{2}\right) \Gamma\left(\frac{s+k}{2}\right) \\
& =\pi^{-\frac{3}{2} s+\frac{1}{2}} 2^{2-s-k} \Gamma\left(\frac{s+1}{2}\right) \Gamma(s+k-1) .
\end{aligned}
$$

Let

$$
f(z)=\sum_{n \geq 1} a_{f}(n) n^{\frac{k-1}{2}} e(n z)
$$

be the Fourier expansion of $f$. Then we have the following relation between $\lambda_{f}(n)$ (Hecke egienvalue) and $a_{f}(m)$ (Fourier coefficient),

$$
a_{f}(n)=a_{f}(1) \lambda_{f}(n)
$$

with

$$
\left|a_{f}(1)\right|^{2}=\frac{(4 \pi)^{k-1}}{(k-1) !} \frac{2 \pi^{2}}{L\left(1, \mathrm{sym}^{2} f\right)} .
$$

For an even level 1 Hecke-Maass form $\phi$ with $n$-th Hecke eigenvalue $\lambda_{f}(n)$. Then one can define the following Rankin-Selberg $L$-function:

$$
L\left(s, \phi \otimes \operatorname{sym}^{2} f\right)=\sum_{m, n \geq 1} \frac{\lambda_{\phi}(m) a_{F}(m, n)}{\left(m n^{2}\right)^{s}}
$$


where

$$
a_{F}(m, n)=\sum_{d \mid(m, n)} \lambda_{F}\left(\frac{m}{d}, 1\right) \lambda_{F}\left(\frac{n}{d}, 1\right) \mu(d)
$$

and

$$
\lambda_{F}(r, 1)=\sum_{s^{2} t=r} \lambda_{f}\left(t^{2}\right)
$$

Let

$$
\begin{aligned}
\Lambda_{\phi, f}(s) & =\pi^{-3 s} \Gamma\left(\frac{s+k-1+i t_{\phi}}{2}\right) \Gamma\left(\frac{s+k-1-i t_{\phi}}{2}\right) \\
& \times \Gamma\left(\frac{s+k+i t_{\phi}}{2}\right) \Gamma\left(\frac{s+k-i t_{\phi}}{2}\right) \Gamma\left(\frac{s+1+i t_{\phi}}{2}\right) \\
& \times \Gamma\left(\frac{s+1-i t_{\phi}}{2}\right) L\left(s, \phi \otimes \operatorname{sym}^{2} f\right)
\end{aligned}
$$

where $\frac{1}{4}+i t_{\phi}^{2}$ is the Laplace eigenvalue of $\phi$. Then $\Lambda_{\phi, f}(s)$ admits analytic continuation to $\mathbb{C}$ as an entire function and satisfies the functional equation

$$
\Lambda_{\phi, f}(s)=\Lambda_{\phi, f}(1-s)
$$

\subsection{Main Results}

\subsubsection{Local large sieve inequalities for $G L(2)$ cusp forms}

The classical theory of large sieve has its genesis in the work of Linnik on the least quadratic non-residue. It was subsequently refined and extended by Bombieri, Gallagher, Renyi, Roth and many others. It is probably best known for its role in partial results toward the Goldbach conjecture (works by Rényi, Bombieri, Vinogradov, and Chen who has the best result to this date). An instance of the large sieve inequality for character sum is the following.

Theorem 1.2.1. For any complex numbers $\left\{a_{n}\right\}, N$ a large positive integer.

$$
\sum_{q \leq Q} \sum_{\substack{\chi \\ \chi \text { primitive }}}\left|\sum_{n=N}^{2 N} a_{n} \chi(n)\right|^{2} \ll\left(N+Q^{2}\right) \sum_{n=N}^{2 N}\left|a_{n}\right|^{2} .
$$


Note that the cardinality of the primitive characters modulo $q, q \leq Q$, is of size $\sim Q^{2}$ and $N$ is the cardinality of the set $\left\{a_{n}\right\}_{n=N}^{2 N}$. It is of crucial importance in application that the two cardinalities are separated, i.e. $Q^{2}+N$ instead of $Q^{2}$ and $N$ intertwining multiplicatively. This feature is indeed what we desire for the large sieve inequality in general.

Holomorphic and Maass cusp forms, being the $G L(2)$ analogues of Dirichlet character, have their corresponding large sieve inequality. For holomorphic cusp forms, it was previously studied by Jutila and Motohasi, and for Maass forms, the most notable contributors are Deshoullier and Iwaniec, Jutila, Luo, Motohashi:

Theorem 1.2.2. (Lemma 8 in [15], [2],[14],[21] and [27]) For any $1 \leq G \leq K,\left\{a_{n}\right\}$ complex numbers,

$$
\begin{gathered}
\sum_{\substack{K \leq k \leq K \\
2 \mid k}} \sum_{f \in H_{k}}\left|\sum_{n=N}^{2 N} a_{n} \lambda_{f}(n)\right|^{2} \\
+\sum_{K \leq t \leq K+G} \sum_{f \in \mathfrak{H}_{t}}\left|\sum_{n=N}^{2 N} a_{n} \lambda_{f}(n)\right|^{2} \\
\ll_{\epsilon}(K N)^{\epsilon}(K G+N) \sum_{n=N}^{2 N}\left|a_{n}\right|^{2}
\end{gathered}
$$

where $H_{k}$ is a basis for the space of holomorphic cusp forms of level 1 and weight $k$ consisting of common egienfunctions for all Hecke operators $T_{n}$ and $\lambda_{f}(n)$ is the $n$-th Hecke eigenvalue of $f$; and $\mathfrak{H}_{t}$ is a basis for the space of Maass forms of level 1 consisting of common egienfunctions for all Hecke operators $T_{n}$ and that $f \in \mathfrak{H}_{t}$ if $f$ has Laplace eigenvalue $\frac{1}{4}+t^{2}$.

These settle the $G L(2)$ large sieve inequalities in the cases of family in integral weight for holomorphic cusp forms and family in the Laplacian eigenvalue for the Maass form. 
In this thesis, the above Theorem is generalized in two respects: The first is that we take level into consideration and the second is that we prove a large sieve type inequality for half-integral weight holomorphic cusp forms.

For integral weight holomorphic cusp forms and Maass forms, we prove that

Theorem 1.2.3. (see [17])Let $M$ be square-free number, $\left\{a_{n}\right\}$ complex numbers,

$$
\begin{gathered}
\sum_{\substack{K \leq k \leq K+G \\
2 \mid k}} \sum_{f \in H_{k, M}^{*}}\left|\sum_{n=N}^{2 N} a_{n} \lambda_{f}(n)\right|^{2} \\
+\sum_{K \leq t \leq K+G} \sum_{f \in \mathfrak{H}_{t, M}^{*}}\left|\sum_{n=N}^{2 N} a_{n} \lambda_{f}(n)\right|^{2} \\
\ll_{\epsilon}(M N K)^{\epsilon}(K G M+N) \sum_{n=N}^{2 N}\left|a_{n}\right|^{2}
\end{gathered}
$$

where $H_{k, M}^{*}$ is a Hecke basis for the space of new forms of weight $k$ and level $M, \mathfrak{H}_{t, M}^{*}$ is a Hecke basis for the space of new Maass forms with Laplace eigenvalue $\frac{1}{4}+t^{2}$ and level $M$ and $\lambda_{f}(n)$ is the $n$-th Hecke eigenvalue of $f$.

In the half-integral weight case, we have

Theorem 1.2.4. (see [17])For any natural number $M$ divisible by $4,\left\{a_{n}\right\}$ complex numbers,

$$
\sum_{K \leq k \leq K+G} \sum_{f \in B_{k . M}}\left|\sum_{n=N}^{2 N} a_{n} \rho_{f}(n)\right|^{2} \ll_{\epsilon}(M N K)^{\epsilon}(K G M+N) \sum_{n=N}^{2 N}\left|a_{n}\right|^{2} .
$$

Here $B_{k, M}$ is an orthonormal basis (with respect to the Petersson inner product defined above) of the space of holomorphic cusp forms of weight $k$ with level $M$ and $\rho_{f}(n)$ as in Theorem 2.4. 


\subsubsection{Mean value of symmetric square $L$-functions}

Some of the simplest examples of higher rank $L$-functions are the symmetric power $L$ functions. The first detailed study of the symmetric square $L$-functions associated to holomorphic cusp forms was done in 1973 by G. Shimura (see [29]). In this thesis, we investigate the second moment of symmetric square $L$-functions. Namely, we study the sum

$$
\sum_{K \leq k \leq K+G} \sum_{f \in H_{k}} L^{2}\left(\frac{1}{2}, \mathrm{sym}^{2} f\right) .
$$

Ideally one wishes to show

$$
\sum_{f \in H_{k}} L^{2}\left(\frac{1}{2}, \operatorname{sym}^{2} f\right) \ll_{\epsilon, t} K^{1+\epsilon}
$$

seems out of reach we manage to prove

Theorem 1.2.5. For $K^{\frac{1}{3}} \leq G \leq K$,

$$
\sum_{K \leq k \leq K+G} \sum_{f \in H_{k}} L^{2}\left(\frac{1}{2}, \operatorname{sym}^{2} f\right) \ll_{t, \epsilon} K^{1+\epsilon} G .
$$

This improves on the previous result of W. Luo ([23]) where $G$ can be taken to be taken to be $K^{1 / 2} \leq G \leq K$.

\subsubsection{First moment of Rankin-Selberg $L$-functions.}

There has been a lot of work on the mean value of Rankin-Selberg $L$-function. For example, Li established in [20] the sharp bound for the mean value of $G L(3) \times$ $G L(2)$ Rankin-Selberg $L$-functions when the $G L(2)$ forms vary in a range of spectral parameter. In my dissertation I study the mean value of $G L(3) \times G L(2)$ RankinSelberg $L$-functions for a fixed $G L(2)$ cusp form and the $G L(3)$ forms coming form a family of self-dual forms. More precisely, we prove that 
Theorem 1.2.6. Let $\phi$ a fixed Hecke-Maass form, then

$$
\sum_{\substack{k \leq k \leq K+G \\ k \text { even }}} \sum_{f \in H_{k}} \frac{L\left(\frac{1}{2}, \phi \otimes \operatorname{sym}^{2} f\right)}{L\left(1, \operatorname{sym}^{2} f\right)} \ll_{\epsilon} G K^{1+\epsilon}
$$

whenever $K \geq G \geq K^{\frac{1}{3}}$.

Luo and Sarnak proved the case when $G=K$ in [24] as a corollary of their work on Quantum variance of Hecke cusp forms. Theorem 1.2.1 can be viewed as an extension of my result on mean value of the second moment of symmetric square $L$-functions. Indeed, we can view Theorem 1.2.1 as the case when $\phi(z)$ is replaced by the Eisenstein series $E\left(z, \frac{1}{2}+i t\right)$. 


\section{CHAPTER 2 LARGE SIEVE INEQUALITIES}

In this chapter, we prove Theorem 1.2.3 and Theorem 1.2.4. One common ingredient in all the proofs is the embedding method of H. Iwaniec, which we now describe. Take any prime $p$ and for any function $f$ in $B_{k, M}$ (respectively Maass cusp form of level $M$ and for the half integral case $4 \mid M)$, it is automatically a holomorphic cusp form of level $p M$ (respectively Maass cusp form if level $p M$ ). Hence we can embed $B_{k}$ into $B_{k, p M}$ (respectively $B_{M}$ into $B_{k, p M}$ ). We then apply the Petersson trace formula (respectively Kuznetsov trace formula), which gives both diagonal and non-diagonal terms. The management of the diagonal term is straight-forward while the non-diagonal term involves the Bessel functions which traditionally are the source of troubles. The advantage of the embedding method is by choosing $p$ well, we can completely avoid the troublesome transitional range of the Bessel functions.

\subsection{Holomorphic cusp forms of integral weight $k$}

In this section we prove the large sieve inequality for holomorphic cusp forms of integral weight. 


\subsubsection{First manipulations}

Notations as in section 1.2.1. For any small $\epsilon>0$. Assume $K^{1-\delta} \geq G \geq(K M N)^{\delta}$ for some $\delta>0$, otherwise the result is well-known (see section 1.2.1). We assume $M<K^{D}$ for some $D>0$ otherwise the result is trivial. We also assume $N \ll K^{E}$ for some $E>0$ otherwise the result follows easily from direct estimation using Deligne's bound. We will now choose the test function that will smooth our sum. Let $g(x) \in$ $C_{0}^{\infty}(0, \infty)$ such that $\operatorname{supp}(g(x)) \subset\left[\frac{1}{2}, \frac{5}{2}\right], g^{(j)} \ll_{j} 1$ for all $j \geq 0$, and $g(x)=1$ for $x \in[1,2]$. To prove the large sieve inequality, it suffices to bound, for sufficiently large $K$, the sum (we shift the sum for convenience but the adjustment to the original is simple)

$$
S_{M}=\sum_{\substack{k \geq 1 \\ 2 \mid k}} g\left(\frac{k-K}{G}\right) \sum_{f \in B_{k, M}}\left|\sum_{n=N}^{2 N} a_{n} \rho_{f}(n)\right|^{2}
$$

Choose prime $p$ in the interval

$$
\left[(K M N)^{\epsilon} \max \left\{\frac{N}{M K G}, 1\right\}, 2(K M N)^{\epsilon} \max \left\{\frac{N}{M K G}, 1\right\}\right] .
$$

Such prime exits by the Bertrand postulate.

For any $f \in H_{k, M}, f$ is holomorphic cusp forms of weight $k$ and level $p M$. On the other hand,

$$
\begin{aligned}
& \frac{1}{\operatorname{vol}\left(X_{0}(p M)\right)} \int_{X_{0}(p M)} f(z) \overline{f(z)} y^{k} \frac{d x d y}{y^{2}} \\
= & \frac{1}{\operatorname{vol}\left(X_{0}(M)\right)\left[\Gamma_{0}(p M): \Gamma_{0}(M)\right]} \int_{X_{0}(p M)} f(z) \overline{f(z)} y^{k} \frac{d x d y}{y^{2}} \\
= & \frac{1}{\operatorname{vol}\left(X_{0}(M)\right)} \int_{X_{0}(M)} f(z) \overline{f(z)} y^{k} \frac{d x d y}{y^{2}}=1 .
\end{aligned}
$$

Also if $f$ is an Hecke eigenform for all $T_{q}$ with $(q, M)=1$, then $f$ is an eigenform for all $T_{q}$ with $\left.(q, p M)=1\right)$. Hence we can embed $H_{k, M}$ into $H_{k, p M}$. 
By positivity, $S_{M} \leq S_{p M}$. Applying the Petersson trace formula to $S_{p M}, S_{p M}=$

$$
\begin{aligned}
& \operatorname{vol}\left(X_{0}(p M)\right) \sum_{k \text { even }} g\left(\frac{k-K}{G}\right)(k-1) \sum_{n=N}^{2 N}\left|a_{n}\right|^{2} \\
& +\sum_{N \leq n_{1}, n_{2} \leq 2 N} a_{n_{1}} \overline{a_{n_{2}}} \operatorname{vol}\left(X_{0}(p M)\right) \sum_{c \equiv 0(p M)} \frac{S\left(n_{1}, n_{2} ; c\right)}{c} 2 \pi \times \\
& \sum_{k \text { even }} i^{k} g\left(\frac{k-K}{G}\right)(k-1) J_{k-1}\left(\frac{4 \pi \sqrt{n_{1} n_{2}}}{c}\right) .
\end{aligned}
$$

We call (2.1.1) the diagonal term and (2.1.2) the off-diagonal term.

\subsubsection{The diagonal contribution}

The diagonal term contributes at most $\sum_{m=N}^{2 N}\left|a_{m}\right|^{2}$ times

$$
K G(p M)^{1+\epsilon} \ll(K M)^{\epsilon} p K G M \ll(K N M)^{2 \epsilon} \max \{K G M, N\} .
$$

\subsubsection{The off-diagonal contribution}

Since $J_{k-1}(x) \ll\left(\frac{e x}{2 k}\right)^{k-1}$ (see (5.10.2) in [19]) and $\frac{4 \pi \sqrt{n_{1} n_{2}}}{c} \leq \frac{8 \pi N}{c}$, so the contribution from those $c$ with $c>\frac{24 \pi N}{K}$ is exponentially small. Thus it suffices to consider those $c$ less than or equal $\frac{24 \pi N}{K}$. The sum of weighted Bessel function has the following integral representation.

Lemma 2.1.1. For $h \in C_{0}^{\infty}(0, \infty)$, we have

$$
\sum_{\substack{k \geq 1 \\ 2 \mid k}} 2 \pi(-1)^{\frac{k}{2}} J_{k-1}(x) h(k-1)=-2 \pi \int_{-\infty}^{\infty} \hat{h}(t) \sin (x \cos (2 \pi t)) d t
$$

where $\hat{h}(t)=\int_{-\infty}^{\infty} h(y) e(t y) d y$ is the Fourier transform of $h(t)$.

Proof. See Lemma 4.1 of [24]. 


$$
\begin{aligned}
& \text { Let } h(y)=y g\left(\frac{y-(K-1)}{G}\right) \text {, then } \\
& \hat{h}(t)=\int_{-\infty}^{\infty} y g\left(\frac{y-(K-1)}{G}\right) e(y t) d y \\
& =\int_{-\infty}^{\infty}[y-(K-1)] g\left(\frac{y-(K-1)}{G}\right) e(y t) d y \\
& +(K-1) \int_{-\infty}^{\infty} g\left(\frac{y-(K-1)}{G}\right) e(y t) d y \\
& =e((K-1) t) \int_{-\infty}^{\infty} x g\left(\frac{x}{G}\right) e(x t) d x \\
& +(K-1) e((K-1) t) \int_{-\infty}^{\infty} g\left(\frac{x}{G}\right) e(x t) d x \\
& =e((K-1) t) \hat{h}_{1}(t)+(K-1) e((K-1) t) \hat{h}_{2}(t)
\end{aligned}
$$

where $h_{1}(x)=x g\left(\frac{x}{G}\right)$ and $h_{2}(x)=g\left(\frac{x}{G}\right)$.

Hence,

$$
\begin{aligned}
& \sum_{\substack{k \geq 1 \\
2 \mid k}}(-1)^{\frac{k}{2}} J_{k-1}(x) h(k-1) \\
= & -\sum_{j=1}^{2}(K-1)^{j-1} \int_{-\infty}^{\infty} \hat{h}_{j}(t) e((K-1) t) \sin (x \cos (2 \pi t)) d t .
\end{aligned}
$$

We will show in the next subsection that assuming $\frac{x}{K G} \ll(K M N)^{-\epsilon}$ and $G \leq$ $K^{1-\delta}$ for some $\epsilon, \delta>0$ then for $j=1,2$

\section{Lemma 2.1.2.}

$$
\int_{-\infty}^{\infty} \hat{h}_{j}(t) e((K-1) t) \sin (x \cos (2 \pi t)) d t \ll_{B}(K M N)^{-B}
$$

for all $B>0$. 
Assuming Lemma 2.1.2 and takes $x=\frac{2 \sqrt{n_{1} n_{2}}}{c}, N \leq n_{1}, n_{2} \leq 2 N$, the off-diagonal contribution

$$
\begin{aligned}
& \ll \sum_{N \leq n_{1}, n_{2} \leq 2 N}\left|a_{n_{1}} a_{n_{2}}\right|(p M)^{1+\epsilon} \sum_{\substack{c \equiv 0(p M) \\
c \leq \frac{24 \pi N}{K}}} \frac{\left|S\left(n_{1}, n_{2} ; c\right)\right|}{c}(K M N)^{-B} \\
& \ll(p M)^{1+\epsilon} N^{2+\epsilon}(K M N)^{-B} \sum_{n=N}^{2 N}\left|a_{n}\right|^{2}
\end{aligned}
$$

which is much less the diagonal contribution upon taking, say, $B=2015$. This established the large sieve inequality of holomorphic cusp forms of integral weight.

\subsubsection{Proof of Lemma 2.1.2}

We first prove the following basic Lemma in Fourier analysis.

Lemma 2.1.3. Let $h \in C_{c}^{\infty}(0, \infty)$ be such that supp $h \subset[K, K+G]$ and $h^{(j)} \ll_{j}$ $G^{-j+1}$ for all $j>0$. Then

$$
\hat{h}(t) \ll|t|^{-m} G^{-m+1} K
$$

for all $m>0$.

Proof. For each $m \in \mathbb{N}$, integrating by parts $m$ times

$$
\hat{h}(t)=\left(-\frac{1}{2 \pi i t}\right)^{m} \int_{-\infty}^{\infty} h^{(m)}(x) e^{-2 \pi i x t} d x .
$$

Hence,

$$
|\hat{h}|(t) \leq \frac{1}{(2 \pi|t|)^{m}} \int_{-\infty}^{\infty}\left|h^{(m)}(x)\right| d x \ll|t|^{-m} G^{-m+1} K .
$$

As a corollary,

$$
\int_{|t| \geq \frac{K^{\frac{\epsilon}{2}}}{G}} \hat{h}_{j}(t) e((K-1) t) \sin (x \cos (2 \pi t)) d t
$$


is negligible. With a little bit of algebra, we can consider instead the integrals

$$
\int_{-\frac{K^{\frac{\epsilon}{2}}}{G}}^{\frac{K^{\frac{\epsilon}{2}}}{G}} \hat{h}_{j}(t) e((K-1) t \pm x \cos (2 \pi t)) d t .
$$

Let $\omega_{ \pm}(t)=2 \pi(K-1) t \pm x \cos (2 \pi t)$. We claim that for $|t| \leq \frac{K^{\epsilon / 2}}{G}, x \leq$ $(M N K)^{-\epsilon} K G$, we have

$$
\left|\omega_{ \pm}^{\prime}(t)\right|>\frac{K}{2}
$$

To see this, note that

$$
\omega_{ \pm}^{\prime}(t)=2 \pi(K-1) \mp x \sin (2 \pi t)=2 \pi(K-1) \mp(2 \pi) x t \frac{\sin (2 \pi t)}{2 \pi t} .
$$

Since $\lim _{u \rightarrow 0} \frac{\sin u}{u}=1$ and $G \gg K^{\delta}$, we have for $|t| \leq \frac{K^{\epsilon / 2}}{G}$ that $\left|\frac{\sin (2 \pi t)}{2 \pi t}\right| \leq \frac{3}{2}$.

From which we have

$$
\left|(2 \pi) x t \frac{\sin (2 \pi t)}{2 \pi t}\right| \leq(2 \pi) \frac{3}{2}|x t| \leq(2 \pi) \frac{3}{2}(K N M)^{-\epsilon} K G \frac{K^{\epsilon / 2}}{G}<\frac{K}{2} .
$$

Hence our claim.

Note that

$$
\begin{gathered}
\hat{h}_{1}^{(\nu)}(t)=(2 \pi i)^{\nu} \int_{-\infty}^{\infty} x^{\nu+1} g\left(\frac{x}{G}\right) e(x t) d x \\
=(2 \pi i G)^{\nu} G \int_{-\infty}^{\infty}\left(\frac{x}{G}\right)^{\nu+1} g\left(\frac{x}{G}\right) e(x t) d x \ll G^{\nu+2} .
\end{gathered}
$$

Similarly, $\hat{h}_{2}^{(\nu)}(t) \ll G^{\nu+1}$.

We now apply integrating by parts to the oscillatory integral $\int_{-\frac{K^{\frac{\epsilon}{2}}}{G}}^{\frac{K^{2}}{G}} \hat{h}_{j}(t) e^{\omega_{ \pm}(t)} d t$ $-\frac{K^{\frac{\epsilon}{2}}}{G}$

with $\left|\omega_{ \pm}^{\prime}(t)\right|>\frac{K}{2}, \hat{h}_{j}^{(\nu)}(t) \ll G^{\nu+3-j}$, and $\omega_{ \pm}^{(k)}(t) \ll \frac{x}{K G}$ for $k>1$ to conclude the proof of Lemma 2.1.2. 


\subsection{Holomorphic cusp forms of half integral weight}

In this section we prove the large sieve inequality for holomorphic cusp forms of half integral weight.

\subsubsection{First manipulation}

Notations as in section 1.2.1. For any small $\epsilon>0$. Assume without loss of generality

that $(K M N)^{\delta} \leq G \leq K^{1-\delta}$ for some $1>\delta>0$. Also assume $N \leq K^{D}$ for some $D>0$ otherwise the result is trivial using Cauchy-Schwarz inequality and mean value estimate of the Fourier coefficient of half integral weight holomorphic cusp forms (see P.70 of [9]). Let $g(x) \in C_{0}^{\infty}(0, \infty)$ such that $\operatorname{supp}(g) \subset\left[\frac{1}{2}, \frac{5}{2}\right], g^{(j)} \ll 1$ for all $j \geq 1$, and $g(x)=1$ for $x \in[1,2]$. To prove the large sieve inequality, it suffices to bound, for sufficiently large $K$, the sum (we shift the sum for convenience but the adjustment to the original is simple)

$$
S_{M}=\sum_{\substack{k \geq 1 \\ k-\frac{1}{2} \text { even }}} g\left(\frac{k-K}{G}\right) \sum_{f \in B_{k, M}}\left|\sum_{n=N}^{2 N} a_{n} \rho_{f}(n)\right|^{2} .
$$

Choose a prime $p$ such that

$$
p \in\left[(K M N)^{\epsilon} \max \left\{\frac{N}{M K G}, 1\right\}, 2(K M N)^{\epsilon} \max \left\{\frac{N}{M K G}, 1\right\}\right] .
$$

For the same reason as was presented in 2.1.1

$$
S_{M} \leq \sum_{\substack{k \geq 1 \\ k-\frac{1}{2} \text { even }}} g\left(\frac{k-K}{G}\right) \sum_{f \in B_{k, p M}}\left|\sum_{n=N}^{2 N} a_{n} \rho_{f}(n)\right|^{2} .
$$


By Petersson formula,

$$
\begin{aligned}
& \sum_{\substack{k \geq 1 \\
k-\frac{1}{2} \text { even }}} g\left(\frac{k-K}{G}\right) \sum_{f \in B_{k, p M}}\left|\sum_{n=N}^{2 N} a_{n} \rho_{f}(n)\right|^{2} \\
= & \operatorname{vol}\left(X_{0}(p M)\right) \sum_{\substack{k \geq 1 \\
k-\frac{1}{2} \text { even }}} g\left(\frac{k-K}{G}\right) \sum_{\substack{N \leq n_{1}, n_{2} \leq 2 N\\
}} a_{n_{1}} \overline{a_{n_{2}}}\left\{(k-1) \delta_{n_{1} n_{2}}\right. \\
+ & \left.2 \pi i^{-k}(k-1) \sum_{c \equiv 0(p M)} \frac{K_{k}\left(n_{1}, n_{2} ; c\right)}{c} J_{k-1}\left(\frac{4 \pi \sqrt{n_{1} n_{2}}}{c}\right)\right\}
\end{aligned}
$$

where we define for each complex number $z \neq 0$ and real number $\nu$,

$$
z^{\nu}=|z|^{\nu} \exp (i \nu \arg z) \text { with }-\pi<\arg z \leq \pi
$$

\subsubsection{The diagonal contribution}

The diagonal term

$$
\operatorname{vol}\left(X_{0}(p M)\right) \sum_{\substack{k \geq 1 \\ k-\frac{1}{2} \text { even }}}(k-1) g\left(\frac{k-K}{G}\right) \sum_{m=N}^{2 N}\left|a_{m}\right|^{2}
$$

contributes at most $\sum_{m=N}^{2 N}\left|a_{m}\right|^{2}$ times

$$
K G(p M)^{1+\epsilon} \ll(K M)^{\epsilon} p K G M \ll(K N M)^{2 \epsilon} \max \{K G M, N\} .
$$

\subsubsection{The off-diagonal contribution}

Since $J_{k-1}(x) \ll\left(\frac{e x}{2 k}\right)^{k-1}$ (see (5.10.2) in [19]) and $\frac{4 \pi \sqrt{n_{1} n_{2}}}{c} \leq \frac{8 \pi N}{c}$, so the contribution from those $c$ with $c>\frac{24 \pi N}{K}$ is exponentially small. Thus it suffices to consider those $c$ less than or equal to $\frac{24 \pi N}{K}$, i.e. we can consider instead

$$
\operatorname{vol}\left(X_{0}(p M)\right) \sum_{\substack{k \geq 1 \\ k-\frac{1}{2} \text { even }}} g\left(\frac{k-K}{G}\right) \sum_{N \leq n_{1}, n_{2} \leq 2 N} a_{n_{1}} \overline{a_{n_{2}}} \times
$$




$$
\left\{2 \pi i^{-k}(k-1) \sum_{\substack{c \equiv 0(p M) \\ c \leq \frac{24 \pi N}{K}}} \frac{K_{k}\left(n_{1}, n_{2} ; c\right)}{c} J_{k-1}\left(\frac{4 \pi \sqrt{n_{1} n_{2}}}{c}\right)\right\} .
$$

Note that we have the following integral representation of the $J-$ Bessel function (see P.231 of [30]): For $l \in \mathbb{N} \cup\{0\}$,

$$
J_{l+\frac{1}{2}}(x)=\int_{-\frac{1}{2}}^{\frac{1}{2}} e\left(\left(l+\frac{1}{2}\right) t\right) e^{-i x \sin 2 \pi t} d t+\frac{(-1)^{l}}{\pi} \int_{0}^{\infty} e^{-\left(l+\frac{1}{2}\right) t-x \sinh t} d t .
$$

Hence, (2.2.1)

$$
\begin{aligned}
= & 2 \operatorname{vol}\left(X_{0}(p M)\right) \sum_{\alpha=1}^{2} \sum_{\substack{c \equiv 0(p M) \\
c \leq \frac{24 \pi N}{K}}} \sum_{\substack{K \\
\text { n }}} \sum_{\substack{\text { even } \\
l \geq 2}} \frac{K_{l+\frac{1}{2}}\left(n_{1}, n_{2} ; c\right)}{c} a_{n_{1}} \overline{a_{n_{2}}} \times \\
& i^{-\left(l+\frac{1}{2}\right)}\left(l-\frac{1}{2}\right) g\left(\frac{l+\frac{1}{2}-K}{G}\right) I_{l, \alpha}\left(\frac{4 \pi \sqrt{n_{1} n_{2}}}{c}\right)
\end{aligned}
$$

where

$$
\begin{aligned}
& I_{l, 1}(x)=\pi \int_{-\frac{1}{2}}^{\frac{1}{2}} e\left(\left(l-\frac{1}{2}\right) t\right) e^{-i x \sin 2 \pi t} d t \text { and } \\
& I_{l, 2}(x)=\int_{0}^{\infty} e^{-\left(l-\frac{1}{2}\right) t-x \sinh t} d t .
\end{aligned}
$$

Opening the twisted Kloosterman sum (see 1.1.1 for the equation of $K_{k}\left(n_{1}, n_{2} ; c\right)$ ) and by our definition of $z^{\nu},(2.2 .1)=$

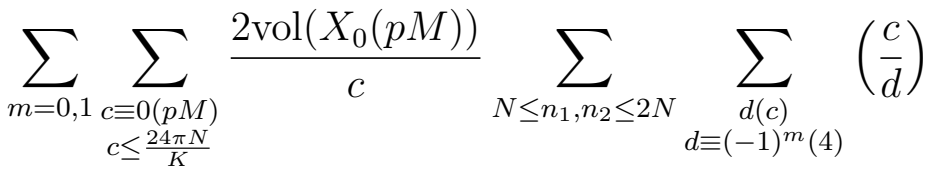

$$
\begin{aligned}
& \times e\left(\frac{n_{1} d+n_{2} \bar{d}}{c}\right) a_{n_{1}} \overline{a_{n_{2}}} \sum_{\substack{l \text { even } \\
l \geq 2}}\left(l-\frac{1}{2}\right) i^{-m(2 l+1)-\left(l+\frac{1}{2}\right)} \times \\
& g\left(\frac{l+\frac{1}{2}-K}{G}\right) I_{l, 1}\left(\frac{4 \pi \sqrt{n_{1} n_{2}}}{c}\right)+
\end{aligned}
$$




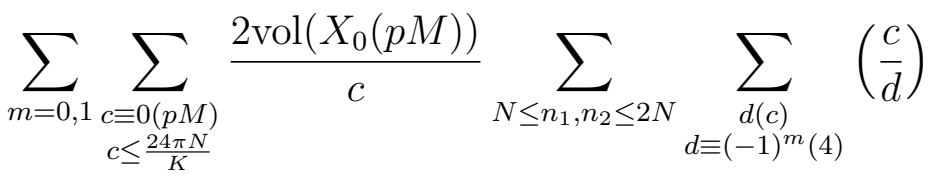

$$
\begin{aligned}
& \times e\left(\frac{n_{1} d+n_{2} \bar{d}}{c}\right) a_{n_{1}} \overline{a_{n_{2}}} \sum_{\substack{l \text { even } \\
l \geq 2}}\left(l-\frac{1}{2}\right) i^{-m(2 l+1)-\left(l+\frac{1}{2}\right)} \\
& \times g\left(\frac{l+\frac{1}{2}-K}{G}\right) I_{l, 2}\left(\frac{4 \pi \sqrt{n_{1} n_{2}}}{c}\right) .
\end{aligned}
$$

We will first deal with (2.2.3), first of all $I_{l, 2}(x)$ can be evaluated explicitly with an error term. Let $\omega(t)=-\left(l-\frac{1}{2}\right) t-x \sinh t$, integrating by parts, we have

$$
I_{l, 2}(x)=\int_{0}^{\infty} e^{\omega(t)} d t=\frac{-1}{l-\frac{1}{2}+x}+\int_{0}^{\infty} e^{\omega(t)} \frac{\omega^{\prime \prime}(t)}{\left[\omega^{\prime}(t)\right]^{2}} d t .
$$

Applying integrating by parts repeatedly, we can show that $I_{l, 2}(x)$ is a sum of

$$
\frac{x^{a}}{\left(l-\frac{1}{2}+x\right)^{b+1+a}}
$$

for some non-negative natural numbers $a, b$, with an error term $O\left(x^{-A}\right)$ for all $A>0$.

The contribution of the error term $O\left(x^{-A}\right)$ is

$$
\begin{aligned}
& \ll p M K G \sum_{\substack{c \equiv 0(p M) \\
c \leq \frac{24 \pi N}{K}}} c^{A}\left(\sum_{n=N}^{2 N} \frac{\left|a_{n}\right|}{n^{A / 2}}\right)^{2} \ll \frac{p M K G(p M)^{A}}{N^{A-1}} \sum_{t=1}^{\frac{24 \pi N}{K p M}} t^{A} \sum_{n=N}^{2 N}\left|a_{n}\right|^{2} \\
& \ll \frac{N^{2}}{K^{A-1}} \sum_{n=N}^{2 N}\left|a_{n}\right|^{2} .
\end{aligned}
$$

Since we assume a prior that $N \ll K^{D}$ for some $D>0$, by taking $A=B+1$, we have the term above $\ll N \sum\left|a_{n}\right|^{2}$. Hence to show $(2.2 .3) \ll(M K N)^{\epsilon}(K G M+$ N) $\sum_{n=N}^{2 N}\left|a_{n}\right|^{2}$, it suffices to show

\section{Lemma 2.2.1.}

$$
\operatorname{vol}\left(X_{0}(p M)\right) \sum_{m=0,1} \sum_{\substack{c \equiv 0(p M) \\ c \leq \frac{24 \pi N}{K}}} \frac{1}{c} \sum_{\substack{d(c) \\ d \equiv(-1)^{m}(4)}}\left(\frac{c}{d}\right) \sum_{j= \pm 1} i^{-1+\frac{j}{2}}
$$




$$
\begin{aligned}
& \sum_{\substack{l \text { even } \\
l \geq 2}}\left(l-\frac{1}{2}\right) i^{l(j-2)} g\left(\frac{l+\frac{1}{2}-K}{G}\right) \\
& \times \quad \sum_{N \leq n_{1}, n_{2} \leq 2 N} e\left(\frac{n_{1} \bar{d}+n_{2} d}{c}\right) \frac{a_{n_{1}} \overline{a_{n_{2}}}\left(\frac{4 \pi \sqrt{n_{1} n_{2}}}{c}\right)^{a}}{\left(l-\frac{1}{2}+\frac{4 \pi \sqrt{n_{1} n_{2}}}{c}\right)^{b+1+a}} \\
& \ll(M K N)^{\epsilon}(K G M+N) \sum_{n=N}^{2 N}\left|a_{n}\right|^{2}
\end{aligned}
$$

for all non-negative natural number $a, b$.

To prove Lemma 2.2.1 we need the following hybrid large sieve inequality.

Theorem 2.2.2. For any complex numbers $\left\{a_{n}\right\}, M<n \leq M+N$, let $x_{1} \cdots, x_{R}$ be real numbers which are distinct $\bmod 1$. Let $\delta=\min _{\substack{r, s \\ r \neq s}}\left\|x_{r}-x_{s}\right\|$, where if $R \geq 2$, $\|x\|:=\min _{k \in \mathbb{Z}}|x-k| ; \delta:=\infty$, if $R=1$. Then

$$
\sum_{r=1}^{R}\left|\sum_{n=M+1}^{M+N} a_{n} e\left(n x_{r}\right)\right|^{2} \leq\left(\pi N+\delta^{-1}\right) \sum_{n=N+1}^{M+N}\left|a_{n}\right|^{2}
$$

Proof. See Theorem 2.1 in [28]

Corollary 2.2.3. Notations as before. Let $Q, s$ be natural numbers. Then

$$
\sum_{\substack{q \leq Q \\ q \equiv 0(s)}} \sum_{\substack{(l, q)=1 \\ 0<l<q}}\left|\sum_{n=M+1}^{M+N} a_{n} e\left(\frac{n l}{q}\right)\right|^{2} \ll\left(N+\frac{Q^{2}}{s}\right) \sum_{n=M+1}^{M+N}\left|a_{n}\right|^{2} .
$$

Proof. For $(l, q)=1,0<l<q, q \leq Q, q \equiv 0(s), q=s v, q^{\prime}=s v^{\prime}$, let $x_{l, q}=\frac{l}{q}$. Then for each $q \neq q^{\prime}$,

$$
\left\|x_{l, q}-x_{l^{\prime}, q^{\prime}}\right\|=\left\|\frac{l}{q}-\frac{l^{\prime}}{q^{\prime}}\right\|=\left\|\frac{l}{s v}-\frac{l^{\prime}}{s v^{\prime}}\right\|
$$

for some $v, v^{\prime} \in \mathbb{N}$. Hence, (since $s v^{\prime}, s v \leq Q$ )

$$
\left\|x_{l, q}-x_{l^{\prime}, q^{\prime}}\right\| \geq \frac{1}{s v v^{\prime}} \geq \frac{1}{\frac{Q^{2}}{s}}
$$


Hence taking $\delta=\frac{s}{Q^{2}}$ in Theorem 2.2.2,

$$
\sum_{\substack{q \leq Q \\ q \equiv 0(s)}} \sum_{\substack{(l, q)=1 \\ 0<l<q}}\left|\sum_{n=M+1}^{M+N} a_{n} e\left(\frac{n l}{q}\right)\right|^{2} \ll\left(N+\frac{Q^{2}}{s}\right) \sum_{n=M+1}^{M+N}\left|a_{n}\right|^{2} .
$$

Proposition 2.2.4. Notations as before. For $a_{n} \in \mathbb{C}$, let

$$
\sum_{n=1}^{\infty}\left|a_{n}\right|<\infty
$$

Then, for $T \geq 1$,

$$
\sum_{\substack{q \leq Q \\ q \equiv 0(s)}} \sum_{\substack{(l, q)=1 \\ 0<l<q}} \int_{-T}^{T}\left|\sum_{n=1}^{\infty} a_{n} e\left(\frac{n l}{q}\right) n^{-i t}\right|^{2} d t \ll \sum_{n=1}^{\infty}\left(\frac{T Q^{2}}{s}+n\right)\left|a_{n}\right|^{2} .
$$

Proof. See the proof of Theorem 5.1 in [28].

We define, for any complex sequence $\left\{b_{n}\right\}_{N \leq n \leq 2 N}$,

$$
B\left(\left\{b_{n}\right\}, c, N, t\right)=\sum_{m=0,1} \sum_{\substack{d(c) \\ d \equiv(-1)^{m}(4)}}\left|\sum_{n=N}^{2 N} b_{n} e\left(\frac{n d}{c}\right) n^{-i t}\right|^{2} .
$$

We need to show for all non-negative $a, b$ that

\section{Lemma 2.2.5.}

$$
\begin{aligned}
& \operatorname{vol}\left(X_{0}(p M)\right) \sum_{\substack { m=0,1 \\
\begin{subarray}{c}{c \equiv 0(p M) \\
c \leq \frac{24 \pi N}{K}{ m = 0 , 1 \\
\begin{subarray} { c } { c \equiv 0 ( p M ) \\
c \leq \frac { 2 4 \pi N } { K } } }\end{subarray}} \frac{1}{c} \sum_{\substack{d(c) \\
d \equiv(-1)^{m}(4)}}\left(\frac{c}{d}\right) \times \\
& \sum_{N \leq n_{1}, n_{2} \leq 2 N} e\left(\frac{n_{1} \bar{d}+n_{2} d}{c}\right) a_{n_{1}} \overline{a_{n_{2}}} \frac{\left(\frac{4 \pi \sqrt{n_{1} n_{2}}}{c}\right)^{a}}{\left(l-\frac{1}{2}+\frac{4 \pi \sqrt{n_{1} n_{2}}}{c}\right)^{b+1+a}} \\
& \ll\left(\frac{N}{K^{b+2}}+\frac{p M}{K^{b}}\right) \sum\left|a_{n}\right|^{2}
\end{aligned}
$$


Proof. We follow the argument on P.256 of [2]. The above sum remains unchanged if a smooth weight $h\left(\frac{\sqrt{n_{1} n_{2}}}{N}\right)$ is attached to each term provided that

$$
h(x)=\left\{\begin{array}{l}
1 \text { if } x \in(1,2] \\
0 \text { if } x \notin\left(\frac{1}{2}, 3\right] .
\end{array}\right.
$$

In what follows we demand $h(x)$ to be of $C^{\infty}$ class. Then

$$
\frac{h(x)}{\left(l-\frac{1}{2}+\frac{4 \pi}{c} x N\right)^{b+1+a}}=\frac{1}{2 \pi i} \int_{1-i \infty}^{1+i \infty} H(s) x^{-s} d s
$$

with

$$
H(s)=\int_{0}^{\infty} h(x) \frac{1}{\left(l-\frac{1}{2}+\frac{4 \pi}{c} x N\right)^{b+1+a}} x^{i t} d x .
$$

Hence, $(2.2 .5)=$

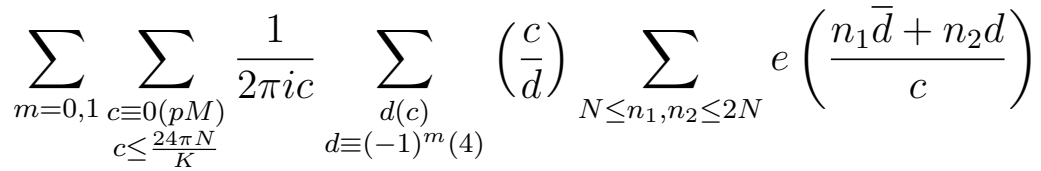

$$
\begin{aligned}
& \times \operatorname{vol}\left(X_{0}(p M)\right) a_{n_{1}} \overline{a_{n_{2}}}\left(\frac{4 \pi \sqrt{n_{1} n_{2}}}{c}\right)^{a} \int_{1-i \infty}^{1+i \infty} H(s)\left(\frac{\sqrt{n_{1} n_{2}}}{N}\right)^{-s} d s \\
& =\frac{\operatorname{vol}\left(X_{0}(p M)\right)}{2 \pi i} \sum_{m=0,1} \sum_{\substack{c \equiv 0(p M) \\
c \leq \frac{24 \pi N}{K}}} \frac{(4 \pi)^{a}}{c^{a+1}} \sum_{\substack{d(c) \\
d \equiv(-1)^{m}(4)}}\left(\frac{c}{d}\right) \int_{1-i \infty}^{1+i \infty} H(s) \times \\
& \left(\sum_{n_{1}=N}^{2 N} a_{n_{1}} n_{1}^{\frac{a}{2}}\left(\frac{n_{1}}{N}\right)^{-\frac{s}{2}} e\left(\frac{n_{1} \bar{d}}{c}\right)\right) \\
& \times\left(\sum_{n_{2}=N}^{2 N} \overline{a_{n_{2}}} n_{2}^{\frac{a}{2}}\left(\frac{n_{2}}{N}\right)^{-\frac{s}{2}} e\left(\frac{n_{2} d}{c}\right)\right) d s
\end{aligned}
$$




$$
\begin{aligned}
& \ll \sum_{\substack{c \equiv 0(p M) \\
c \leq \frac{24 \pi N}{K}}} \frac{p M N}{c^{a+1}} \int_{-\infty}^{\infty} B\left(\left\{a_{n} n^{\frac{a-1}{2}}\right\}, c, N, t\right)|H(1+2 i t)| d t \\
& \leq \sum_{\substack{c \equiv 0(p M) \\
c \leq \frac{24 \pi N}{K}}} \frac{p M N}{c^{a+1}} \int_{-1}^{1} B\left(\left\{a_{n} n^{\frac{a-1}{2}}\right\}, c, N, t\right)|H(1+2 i t)| d t \\
& +\sum_{\substack{c \equiv 0(p M) \\
c \leq \frac{24 \pi N}{K}}} \frac{p M N}{c^{a+1}} \sum_{\nu \geq 0} \int_{J_{\nu}} B\left(\left\{a_{n} n^{\frac{a-1}{2}}\right\}, c, N, t\right)|H(1+2 i t)| d t
\end{aligned}
$$

where $J_{\nu}=\left[-2^{\nu+1},-2^{\nu}\right] \cup\left[2^{\nu}, 2^{\nu+1}\right]$. Integrating by parts,

$$
H(1+i t) \ll\left\{\begin{array}{l}
(1+|t|)^{-\frac{1}{2}} \frac{1}{\left(l-\frac{1}{2}+\frac{4 \pi N}{c}\right)^{b+1+a}} \\
t^{-2} \frac{1}{\left(l-\frac{1}{2}+\frac{4 \pi N}{c}\right)^{b+1+a}} \text { for }|t|>\frac{16 \pi N}{c} .
\end{array}\right.
$$

Then, (2.2.5)

$$
\begin{aligned}
& \ll \sum_{\substack{c \equiv 0(p M) \\
c \leq \frac{24 \pi N}{K}}} \frac{p M N}{c^{a+1}} \int_{-1}^{1} B\left(\left\{a_{n} n^{\frac{a-1}{2}}\right\}, c, N, t\right)\left(\frac{c}{N}\right)^{b+1+a} d t+\sum_{v=0}^{\infty} \sum_{\substack{c \equiv 0(p M) \\
c \leq \frac{24 \pi N}{K}}} \\
& \times \frac{p M N}{c^{a+1}} \int_{J_{\nu}} B\left(\left\{a_{n} n^{\frac{a-1}{2}}\right\}, c, N, t\right) \min \left\{\frac{1}{\sqrt{1+2^{v}}}, 2^{-2 v}\right\}\left(\frac{c}{N}\right)^{b+1+a} d t
\end{aligned}
$$

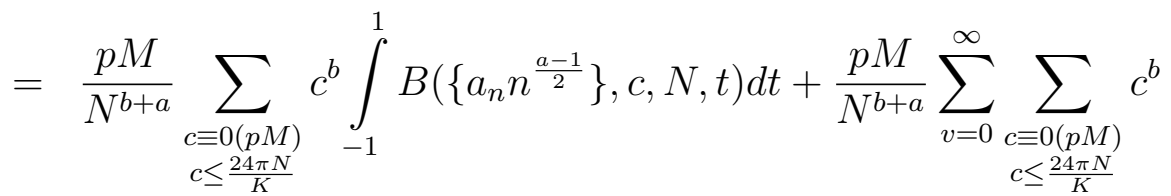

$$
\begin{aligned}
& \times \quad B\left(2^{\nu}, c\right) \min \left\{\frac{1}{\sqrt{1+2^{v}}}, 2^{-2 v}\right\}
\end{aligned}
$$

where

$$
B(T, c)=\int_{[T, 2 T] \cup[-2 T,-T]} B\left(\left\{a_{n} n^{\frac{a-1}{2}}\right\}, c, N, t\right) d t .
$$


By the Abel summation formula,

$$
\begin{aligned}
& \frac{p M}{N^{b+a}} \sum_{\substack{c \equiv 0(p M) \\
c \leq \frac{24 \pi N}{K}}} c^{b} B(T, c)=\frac{(p M)^{b+1}}{N^{a+b}} \sum_{u=1}^{\frac{24 \pi N}{K p M}} u^{b} B(T, p M u) \\
\ll & \frac{(p M)^{n+1}}{N^{a+b}}\left(\sum_{u=1}^{\frac{24 \pi N}{K p M}} B(T, p M u)\right)\left(\frac{24 \pi N}{K p M}\right)^{b} \\
+ & \int_{1}^{\frac{24 \pi N}{K p M}} x^{b-1}\left(\sum_{u=1}^{x} B(T, p M u)\right) d x \frac{(p M)^{b+1}}{N^{a+b}} \\
\ll & \frac{p M}{N^{a} K^{b}} \sum_{\substack{c \equiv 0(p M) \\
c \leq \frac{24 \pi N}{K}}} B(T, c)+\frac{(p M)^{b+1}}{N^{a+b}} \int_{1}^{\frac{24 \pi N}{K p M}} x^{b-1}\left(\sum_{\substack{c \equiv 0(p M) \\
c \leq x p M}} B(T, c)\right) d x
\end{aligned}
$$

By Proposition 2.2.4,

$$
\begin{aligned}
\sum_{\substack{c \equiv 0(p M) \\
c \leq x p M}} B(T, c) \ll \sum_{n=N}^{2 N}\left(T x^{2} p M+n\right)\left|a_{n}\right|^{2} n^{a-1} \\
\ll\left(T x^{2} p M N^{a-1}+N^{a}\right) \sum_{n=N}^{2 N}\left|a_{n}\right|^{2} .
\end{aligned}
$$

Hence,

$$
\begin{aligned}
& \frac{p M}{N^{b+a}} \sum_{\substack{c \equiv 0(p M) \\
c \leq \frac{24 \pi N}{K}}} c^{b} B(T, c) \ll \frac{p M}{N^{a} K^{b}}\left(T \frac{N^{2}}{K^{2} p M} N^{a-1}+N^{a}\right) \sum_{n=N}^{2 N}\left|a_{n}\right|^{2} \\
+ & \frac{(p M)^{b+1}}{N^{a+b}} \int_{1}^{\frac{24 \pi N}{K p M}} x^{b-1}\left(T x^{2} p M N^{a-1}+N^{a}\right) d x \sum_{n=N}^{2 N}\left|a_{n}\right|^{2} \\
\ll & \left(\frac{T N}{K^{b+2}}+\frac{p M}{K^{b}}\right) \sum_{n=N}^{2 N}\left|a_{n}\right|^{2} \\
+ & \frac{(p M)^{b+1}}{N^{a+b}}\left[T p M N^{a-1}\left(\frac{N}{K p M}\right)^{b+2}+N^{a}\left(\frac{N}{K p M}\right)^{b}\right] \sum_{n=N}^{2 N}\left|a_{n}\right|^{2} \\
\ll & {\left[\frac{T N}{K^{b+2}}+\frac{p M}{K^{b}}\right] \sum_{n=N}^{2 N}\left|a_{n}\right|^{2} . }
\end{aligned}
$$


Hence, (2.2.5)

$$
\ll\left[\frac{N}{K^{b+2}}+\frac{p M}{K^{b}}\right] \sum_{n=N}^{2 N}\left|a_{n}\right|^{2} .
$$

Proof. (of Lemma 2.2.5)

By Lemma 2.2.5, (2.2.4)

$$
\begin{aligned}
& \ll \sum_{k \geq 1} g\left(\frac{2 k-K+\frac{1}{2}}{G}\right)\left(2 k-\frac{1}{2}\right)\left(\frac{N}{K^{2}}+p M\right) \sum_{m=N}^{2 N}\left|a_{m}\right|^{2} \\
& \ll K G\left(\frac{N}{K^{2}}+p M\right) \sum_{m=N}^{2 N}\left|a_{m}\right|^{2} \ll(M K N)^{\epsilon}(K G M+N) \sum_{m=N}^{2 N}\left|a_{m}\right|^{2} .
\end{aligned}
$$

Thus concludes the proof of Lemma 2.2.1

We now develop the appropriate Neumann theory for (2.2.2). We start with

Lemma 2.2.6. For $h \in C_{0}^{\infty}(0, \infty), \alpha \in \mathbb{R}$,

$$
\begin{aligned}
& \sum_{l} h(l) e(\alpha l) \int_{-\frac{1}{2}}^{\frac{1}{2}} e\left(\left(l+\frac{1}{2}\right) t\right) e^{-i x \sin 2 \pi t} d t \\
= & \int_{-\infty}^{\infty} \hat{h}(t) e\left(\frac{\lfloor\alpha-t\rfloor+\alpha-t}{2}\right) e^{-i x \sin 2 \pi(\alpha-t)} d t
\end{aligned}
$$

Proof. Let $g_{x}(t)=e\left(\frac{t}{2}\right) e^{-i x \sin 2 \pi t}$ and

$$
f(t)=\left\{\begin{array}{l}
g_{x}(t) t \in\left(-\frac{1}{2}, \frac{1}{2}\right] \\
0 \text { otherwise }
\end{array}\right.
$$

then, $\int_{-\frac{1}{2}}^{\frac{1}{2}} e(l t) g_{x}(t) d t=\int_{-\infty}^{\infty} e(l t) f(t) d t=\hat{f}(l)$, the Fourier transform of $f$ at $l$. By Poisson summation formula, denoting the Fourier transform by $\mathfrak{F}$,

$$
\sum_{l} h(l) \int_{-\frac{1}{2}}^{\frac{1}{2}} e\left(\left(l+\frac{1}{2}\right) t\right) e^{-i x \sin 2 \pi t} d t
$$




$$
\begin{aligned}
& =\sum_{l} h(l) \hat{f}(l)=\sum_{l} \mathfrak{F}(\hat{h}(-\cdot) * f)(l) \\
& =\sum_{l}(\hat{h}(-\cdot) * f)(l)=\sum_{l} \int_{-\infty}^{\infty} \hat{h}(-t) f(l-t) d t \\
& =\int_{-\infty}^{\infty} \hat{h}(t) \sum_{l} f(l+t) d t .
\end{aligned}
$$

We now have to show

$$
\sum_{l} f(l+t)=e\left(\frac{\lfloor t\rfloor+t}{2}\right) e^{-i x \sin 2 \pi t} .
$$

For each $t \in \mathbb{R}$, if $t=2 n+y$ for some $y \in\left(-\frac{1}{2}, \frac{1}{2}\right]$ and $n \in \mathbb{Z}$, then

$$
\begin{aligned}
& \sum_{l} f(l+t)=f(y)=g_{x}(y)=e\left(\frac{y}{2}\right) e^{-i x \sin 2 \pi y} \\
= & e\left(\frac{2 n+y}{2}\right) e^{-i x \sin 2 \pi(2 n+y)}=e\left(\frac{\lfloor t\rfloor+t}{2}\right) e^{-i x \sin 2 \pi t} ;
\end{aligned}
$$

if $t=2 n+1+y$ for some $y \in\left(-\frac{1}{2}, \frac{1}{2}\right]$ and $n \in \mathbb{Z}$, then

$$
\begin{aligned}
& \sum_{l} f(l+t)=f(y)=g_{x}(y)=e\left(\frac{y}{2}\right) e^{-i x \sin 2 \pi y} \\
= & -e\left(\frac{2 n+1+y}{2}\right) e^{-i x \sin 2 \pi(2 n+1+y)}=e\left(\frac{\lfloor t\rfloor+t}{2}\right) e^{-i x \sin 2 \pi t} .
\end{aligned}
$$

Hence $\sum_{l} f(l+t)=e\left(\frac{\lfloor t\rfloor+t}{2}\right) e^{-i x \sin 2 \pi t}$.

Applying the above to $h_{\alpha}(t)=h(t) e(\alpha t)$, then

$$
\begin{aligned}
& \sum_{l} h(l) e(\alpha l) \int_{-\frac{1}{2}}^{\frac{1}{2}} e\left(\left(l+\frac{1}{2}\right) t\right) e^{-i x \sin 2 \pi t} d t \\
= & \sum_{l} h_{\alpha}(l) \int_{-\frac{1}{2}}^{\frac{1}{2}} e\left(\left(l+\frac{1}{2}\right) t\right) e^{-i x \sin 2 \pi t} d t \\
= & \int_{-\infty}^{\infty} \widehat{h_{\alpha}}(t) e\left(\frac{\lfloor t\rfloor+t}{2}\right) e^{-i x \sin 2 \pi t} d t .
\end{aligned}
$$


Notice that

$$
\begin{gathered}
\widehat{h_{\alpha}}(t)=\int_{-\infty}^{\infty} h(y) e(\alpha y) e(-y t) d y \\
=\int_{-\infty}^{\infty} h(y) e((\alpha-t) y) d y=\hat{h}(\alpha-t) .
\end{gathered}
$$

Hence,

$$
\begin{aligned}
& \sum_{l} h(l) e(\alpha l) \int_{-\frac{1}{2}}^{\frac{1}{2}} e\left(\left(l+\frac{1}{2}\right) t\right) e^{-i x \sin 2 \pi t} d t \\
= & \int_{-\infty}^{\infty} \hat{h}(\alpha-t) e\left(\frac{\lfloor t\rfloor+t}{2}\right) e^{-i x \sin 2 \pi t} d t \\
= & \int_{-\infty}^{\infty} \hat{h}(t) e\left(\frac{\lfloor\alpha-t\rfloor+(\alpha-t)}{2}\right) e^{-i x \sin 2 \pi(\alpha-t)} d t .
\end{aligned}
$$

In particular, take $\alpha=\frac{1}{4}$

$$
\begin{aligned}
& \sum_{l} h(l) e\left(\frac{l}{4}\right) \int_{-\frac{1}{2}}^{\frac{1}{2}} e\left(\left(l+\frac{1}{2}\right) t\right) e^{-i x \sin 2 \pi t} d t \\
= & \sum_{l \text { even }}(-1)^{\frac{l}{2}} h(l) \int_{-\frac{1}{2}}^{\frac{1}{2}} e\left(\left(l+\frac{1}{2}\right) t\right) e^{-i x \sin 2 \pi t} d t \\
+ & i \sum_{l \text { odd }}(-1)^{\frac{l-1}{2}} h(l) \int_{-\frac{1}{2}}^{\frac{1}{2}} e\left(\left(l+\frac{1}{2}\right) t\right) e^{-i x \sin 2 \pi t} d t .
\end{aligned}
$$

Hence

$$
\begin{aligned}
& \sum_{l \text { odd }}(-1)^{\frac{l-1}{2}} h(l) \int_{-\frac{1}{2}}^{\frac{1}{2}} e\left(\left(l+\frac{1}{2}\right) t\right) e^{-i x \sin 2 \pi t} d t \\
= & \operatorname{Im}\left(\int_{-\infty}^{\infty} \hat{h}(t) e\left(\frac{\left\lfloor\frac{1}{4}-t\right\rfloor+\frac{1}{4}-t}{2}\right) e^{-i x \cos 2 \pi t} d t\right) .
\end{aligned}
$$


To get rid of the annoying floor function $\left\lfloor\frac{1}{4}-t\right\rfloor$, note that $\hat{h}$ is essentially supported in $\left[-\frac{1}{G}, \frac{1}{G}\right]$ whenever $h$ is supported in $[K, K+G]$ and its $j$-derivative is majorized by $G^{-j+1}$, i.e. $h(t)=\operatorname{tg}\left(\frac{t-(K-1)}{G}\right)$ satisfies the conditions in Lemma 2.1.3. Thus,

Proposition 2.2.7. Notation as above. For all $A>0$,

$$
\begin{aligned}
& \int_{-\infty}^{\infty} \hat{h}(t) e\left(\frac{\left\lfloor\frac{1}{4}-t\right\rfloor+\frac{1}{4}-t}{2}\right) e^{-i x \cos 2 \pi t} d t \\
= & \int_{-\infty}^{\infty} \hat{h}(t) e\left(\frac{1}{8}-\frac{t}{2}\right) e^{-i x \cos 2 \pi t} d t+O\left(K^{-A}\right) .
\end{aligned}
$$

Proof. Fix $0<\delta<1$ such that $\frac{G^{\delta}}{G}<\frac{1}{4}$,

$$
\begin{aligned}
& \int_{-\infty}^{\infty} \hat{h}(t) e\left(\frac{\left\lfloor\frac{1}{4}-t\right\rfloor+\frac{1}{4}-t}{2}\right) e^{-i x \cos 2 \pi t} d t \\
= & \int_{|t| \leq \frac{G^{\delta}}{G}} \hat{h}(t) e\left(\frac{\left\lfloor\frac{1}{4}-t\right\rfloor+\frac{1}{4}-t}{2}\right) e^{-i x \cos 2 \pi t} d t \\
+ & \int_{|t|>\frac{G^{\delta}}{G}} \hat{h}(t) e\left(\frac{\left\lfloor\frac{1}{4}-t\right\rfloor+\frac{1}{4}-t}{2}\right) e^{-i x \cos 2 \pi t} d t .
\end{aligned}
$$

By Lemma 2.1.3,

$$
\begin{aligned}
& \int_{|t|>\frac{G^{\delta}}{G}} \hat{h}(t) e\left(\frac{\left\lfloor\frac{1}{4}-t\right\rfloor+\frac{1}{4}-t}{2}\right) e^{-i x \cos 2 \pi t} d t \\
\ll & K G^{-m+1}\left(\frac{G^{\delta}}{G}\right)^{-m+1}=K G^{\delta(-m+1)} .
\end{aligned}
$$

Taking $m=\left\lfloor 3 \frac{A+1}{\delta}\right\rfloor+2$,

$$
\int_{|t|>\frac{G^{\delta}}{G}} \hat{h}(t) e\left(\frac{\left\lfloor\frac{1}{4}-t\right\rfloor+\frac{1}{4}-t}{2}\right) e^{-i x \cos 2 \pi t} d t \ll K^{-A} .
$$


On the other hand when $|t| \leq \frac{G^{\delta}}{G} \leq \frac{1}{4},\left\lfloor\frac{1}{4}-t\right\rfloor=0$. Hence,

$$
\begin{aligned}
& \int_{-\infty}^{\infty} \hat{h}(t) e\left(\frac{\left\lfloor\frac{1}{4}-t\right\rfloor+\frac{1}{4}-t}{2}\right) e^{-i x \cos 2 \pi t} d t \\
= & \int_{|t| \leq \frac{G^{\delta}}{G}} \hat{h}(t) e\left(\frac{1}{8}-\frac{t}{2}\right) e^{-i x \cos 2 \pi t} d t+O\left(K^{-A}\right) \\
= & \int_{-\infty}^{\infty} \hat{h}(t) e\left(\frac{1}{8}-\frac{t}{2}\right) e^{-i x \cos 2 \pi t} d t+O\left(K^{-A}\right)
\end{aligned}
$$

by using $\int_{|t|>G^{\delta-1}} \hat{h}(t) e\left(\frac{1}{8}-\frac{t}{2}\right) e^{-i x \cos 2 \pi t} d t=O\left(K^{-A}\right)$.

From this we conclude that

$$
\begin{aligned}
& \sum_{l \text { even }}(-1)^{\frac{l}{2}} h(l-1) \int_{-\frac{1}{2}}^{\frac{1}{2}} e\left(\left(l+\frac{1}{2}\right) t\right) e^{-i x \sin 2 \pi t} d t \\
= & \operatorname{Im}\left(\int_{-\infty}^{\infty} \hat{h}(t) e\left(\frac{1}{8}-\frac{t}{2}\right) e^{-i x \cos 2 \pi t} d t\right)+O\left(K^{-A}\right) .
\end{aligned}
$$

As before,

$$
\hat{h}(t)=e((K-1) t) \hat{h}_{1}(t)+(K-1) e((K-1) t) \hat{h}_{2}(t)
$$

where $h_{1}(x)=x g\left(\frac{x}{G}\right)$ and $h_{2}(x)=g\left(\frac{x}{G}\right)$. Hence

$$
\begin{aligned}
& \sum_{l \text { even }}(-1)^{\frac{l}{2}} h(l-1) \int_{-\frac{1}{2}}^{\frac{1}{2}} e\left(\left(l+\frac{1}{2}\right) t\right) e^{-i x \sin 2 \pi t} d t \\
= & \operatorname{Im}\left(\int_{-\infty}^{\infty} \hat{h}_{1}(t) e\left(\frac{1}{8}+\left(K-\frac{3}{2}\right) t-\frac{x}{2 \pi} \cos 2 \pi t\right) d t\right. \\
+ & \left.(K-1) \int_{-\infty}^{\infty} \hat{h}_{2}(t) e\left(\frac{1}{8}+\left(K-\frac{3}{2}\right) t-\frac{x}{2 \pi} \cos 2 \pi t\right) d t\right) .
\end{aligned}
$$

The rest of the proof is then completely analogous to that of Theorem 1.2.3.

\subsection{Maass cusp forms}

In this section we will prove the large sieve inequality for the Maass cusp forms. 


\subsubsection{First Manipulation}

For any small $\epsilon>0$. Assume without loss of generality that $(K M N)^{\delta} \leq G \leq K^{1-\delta}$ for some $\delta>0$. Also assume $N \leq K^{D}$ for some $D>0$ otherwise the result is trivial using Cauchy-Schwarz inequality and mean value estimate of the Fourier coefficient of Maass cusp forms.

Let $h(t)=e^{-\left(\frac{t-K}{G}\right)^{2}}+e^{-\left(\frac{t+K}{G}\right)^{2}}$. To prove Theorem 1.2.3, it suffices to bound, for sufficiently large $K$, the sum (we shift the sum for convenience but the adjustment to the original is simple)

$$
S_{M}=\sum_{f \in H_{M}} \frac{1}{\cosh \pi t_{f}} h\left(t_{f}\right)\left|\sum_{n=N}^{2 N} a_{n} \rho_{t_{f}}(n)\right|^{2} .
$$

Choose a prime $p$ such that

$$
p \in\left[(K M N)^{\epsilon} \max \left\{\frac{N}{M K G}, 1\right\}, 2(K M N)^{\epsilon} \max \left\{\frac{N}{M K G}, 1\right\}\right] .
$$

Under the assumption that $K^{1-\delta} \geq G$ for some $\delta>0$, if $t \in i \mathbb{R}$ then

$$
h(t) \ll e^{-\frac{K^{2}}{G^{2}}} \leq e^{-K^{2 \delta}} .
$$

Thus the contribution from the contribution from the exceptional eigenvalues is negligible.

We embed $H_{M}$ into $H_{p M}$ and since the contribution from the exceptional eigenvalues is small, we can consider instead

$$
\begin{aligned}
& \sum_{f \in H_{p M}} \frac{1}{\cosh \pi t_{f}} h\left(t_{f}\right)\left|\sum_{n=N}^{2 N} a_{n} \rho_{t_{f}}(n)\right|^{2} \\
+ & \sum_{\mathfrak{a} \in \mathfrak{P}_{p M}} \frac{1}{4 \pi} \int_{-\infty}^{\infty}\left|\sum_{n=N}^{2 N} a_{n} \phi_{\mathfrak{a} \infty}^{\Gamma_{0}(p M)}\left(n, \frac{1}{2}+i r\right)\right|^{2} \frac{h(r) d r}{\cosh \pi r} .
\end{aligned}
$$


Applying Kuznetsov formula (see P.409 of [12])

$$
\begin{aligned}
& \sum_{f \in H_{p M}} \frac{1}{\cosh \pi t_{f}} h\left(t_{f}\right)\left|\sum_{n=N}^{2 N} a_{n} \rho_{t_{f}}(n)\right|^{2} \\
+ & \sum_{\mathfrak{a} \in \mathfrak{P}_{p M}} \frac{1}{4 \pi} \int_{-\infty}^{\infty}\left|\sum_{n=N}^{2 N} a_{n} \phi_{\mathfrak{a} \infty}^{\Gamma_{0}(p M)}\left(n, \frac{1}{2}+i r\right)\right|^{2} \frac{h(r) d r}{\cosh \pi r} \\
= & \sum_{N \leq n_{1}, n_{2} \leq 2 N} a_{n_{1}} \overline{a_{n_{2}}} \operatorname{vol}\left(X_{0}(p M)\right) \times \\
& \left\{\frac{\delta_{n_{1} n_{2}}}{\pi^{2}} \int_{-\infty}^{\infty} r h(r) \tanh \pi r d r+\frac{2 i}{\pi}\right. \\
& \left.\sum_{c \equiv 0(p M)} \frac{S\left(n_{1}, n_{2} ; c\right)}{c} \int_{-\infty}^{\infty} J_{2 i r}\left(\frac{4 \pi \sqrt{n_{1} n_{2}}}{c}\right) r h(r) \frac{d r}{\cosh \pi r}\right\} .
\end{aligned}
$$

\subsubsection{The diagonal contribution}

The diagonal terms contribute at most $\sum_{n=N}^{2 N}\left|a_{n}\right|^{2}$ times

$$
K G(p M)^{1+\epsilon} \ll(K M)^{\epsilon} p K G M \ll(K N M)^{2 \epsilon} \max \{K G M, N\} .
$$

Thus it remains to treat the off-diagonal term.

\subsubsection{The off-diagonal contribution}

Let $C=N K^{\frac{1}{4} \epsilon-1}$, for $c>C$, the integral

$$
\frac{2 i}{\pi} \int_{-\infty}^{\infty} J_{2 i r}\left(\frac{4 \pi \sqrt{n_{1} n_{2}}}{c}\right) \frac{r h(r)}{\cosh \pi r} d r
$$

can be made as small as we wish (see P. 631 of [21]). Hence, it suffices to consider those $c$ less than $N K^{\frac{1}{4} \epsilon-1}$. We have the following asymptotic expansion for $J_{2 i r}(x)$ (P.627 in [21])

$$
J_{2 i r}(x)=\frac{e^{i \omega_{x}(2 r)+\pi r-i \frac{\pi}{4}}}{\pi \sqrt{2}}\left(\sum_{m=0}^{B-1} t_{m}\left(4 r^{2}+x^{2}\right)^{-\frac{m}{2}-\frac{1}{4}}+O\left(\left(4 r^{2}+x^{2}\right)^{-\frac{B}{2}}\right)\right)
$$

where $\omega_{x}(r)=\sqrt{r^{2}+x^{2}}-r \log \left(\frac{r}{x}+\sqrt{\left(\frac{r}{x}\right)^{2}+1}\right)$ and $\sum_{n=0}^{\infty}\left|t_{n}\right| S^{-n}<\infty$ for some $S>0$. We assme $K \geq S$ from now on. 
Using the above asymptotic expansion we will show for $K^{1-\frac{\epsilon}{4}} \ll x \ll \frac{N}{p M}$,

$$
\int_{-\infty}^{\infty} J_{2 i r}(x) r h(r) \frac{d r}{\cosh \pi r} \ll(M K N)^{-10}
$$

Indeed,

$$
\begin{aligned}
& \int_{-\infty}^{\infty} J_{2 i r}(x) r h(r) \frac{d r}{\cosh \pi r} \\
= & \frac{e^{-i \frac{\pi}{4}}}{\sqrt{2} \pi} \sum_{m=0}^{B-1} t_{m} \int_{-\infty}^{\infty} r h(r) \frac{e^{\pi r}}{\cosh \pi r}\left(4 r^{2}+x^{2}\right)^{-\frac{1}{4}-\frac{m}{2}} e^{i \omega_{x}(2 r)} d r \\
+ & O\left(\int_{-\infty}^{\infty} r h(r) \frac{e^{\pi r}}{\cosh \pi r}\left(4 r^{2}+x^{2}\right)^{-\frac{B}{2}} d r\right) .
\end{aligned}
$$

Taking $B=\left\lfloor\frac{\log (M N K)}{\log K} 100\right\rfloor$ and bounding trivially

$$
\int_{-\infty}^{\infty} r h(r) \frac{e^{\pi r}}{\cosh \pi r}\left(4 r^{2}+x^{2}\right)^{-\frac{B}{2}} d r \ll K K^{-B} G \ll(M K N)^{-10} .
$$

For each $0 \leq m \leq B-1$, let

$$
h_{m, x}(r)=r h(r) \frac{e^{\pi r}}{\cosh \pi r}\left(4 r^{2}+x^{2}\right)^{-\frac{1}{4}-\frac{m}{2}}
$$

Then by Leibniz's rule,

$$
\begin{aligned}
& h_{m, x}^{(l)}(r)=\sum_{j=0}^{l}\left(\begin{array}{l}
l \\
j
\end{array}\right)\left[\frac{d^{l-j}}{d r^{l-j}}\left(r h(r) \frac{e^{\pi r}}{\cosh \pi r}\right)\right] \\
& \times\left(\frac{d^{j}}{d r^{j}}\left(4 r^{2}+x^{2}\right)^{-\frac{1}{4}-\frac{m}{2}}\right) .
\end{aligned}
$$

And by induction, for $K \leq r \leq K+G$,

$$
\frac{d^{j}}{d r^{j}}\left(4 r^{2}+x^{2}\right)^{-\frac{1}{4}-\frac{m}{2}} \ll m^{j} K^{-\frac{1}{2}-m-j}
$$

and

$$
\begin{aligned}
& \frac{d^{l-j}}{d r^{l-j}}\left(r h(r) \frac{e^{\pi r}}{\cosh \pi r}\right) \\
= & 2 \frac{d^{l-j}}{d r^{l-j}}(r h(r))-2 \frac{d^{l-j}}{d r^{l-j}}\left(r h(r) \frac{e^{-\pi r}}{\cosh \pi r}\right) \\
\ll & G^{-(l-j)} K .
\end{aligned}
$$


Hence,

$$
\begin{aligned}
& h_{m, x}^{(l)}(r) \ll \sum_{j=0}^{l}\left(\begin{array}{l}
l \\
j
\end{array}\right) m^{j} G^{-l+j} K^{\frac{1}{2}-m-j}=G^{-l} K^{\frac{1}{2}-m} \sum_{j=0}^{l}\left(\begin{array}{l}
l \\
j
\end{array}\right)\left(\frac{G m}{K}\right)^{j} \\
= & G^{-l} K^{\frac{1}{2}-m}\left(\frac{G m}{K}+1\right)^{l} \leq G^{-l} K^{\frac{1}{2}-m} B^{l} .
\end{aligned}
$$

We can now estimate $\int_{-\infty}^{\infty} h_{m, x}(r) e^{i \omega_{x}(2 r)} d r$.

\section{Lemma 2.3.1.}

$$
\int_{-\infty}^{\infty} h_{m, x}(r) e^{i \omega_{x}(2 r)} d r \ll(K M N)^{-10} .
$$

Proof. We first have to estimate the size of $\omega_{x}^{\prime}(2 r)$ in the range $K \leq r \leq K+G$ and $K^{1-\frac{\epsilon}{4}} \ll x:=x_{n_{1}, n_{2}}=\frac{4 \pi \sqrt{n_{1} n_{2}}}{c} \ll \frac{N}{p M}$.

Let $u=\frac{2 r}{x}$, then $\frac{p K M}{N} \ll u \ll K^{\frac{\epsilon}{4}}$ and

$$
\omega_{x}^{\prime}(2 r)=-\log \left(u+\sqrt{u^{2}+1}\right)=-u \frac{\log \left(u+\sqrt{u^{2}+1}\right)}{u}<0 .
$$

We will find a lower bound of $f(u):=\frac{\log \left(u+\sqrt{u^{2}+1}\right)}{u}$ in the given range range.

The derivative

$$
f^{\prime}(u)=\frac{u-\sqrt{u^{2}+1} \log \left(\sqrt{u^{2}+1}+u\right)}{u^{2} \sqrt{u^{2}+1}}:=\frac{h(u)}{u^{2} \sqrt{u^{2}+1}}
$$

has exactly one zero $u_{0}$ in $(0, \infty)$ and $f^{\prime}(u)>0$ for all $0<u<u_{0} ; f^{\prime}(u)<0$ for all $u_{0}<u$. To see this notice that $h^{\prime}(u)=\frac{u \log \left(\sqrt{u^{2}+1}+u\right)}{\sqrt{u^{2}+1}}<0$ for all $u>0$ (hence $h(u)$ is decreasing in $(0, \infty)), \lim _{u \rightarrow 0^{+}} h(u)>0$ and $\lim _{u \rightarrow \infty} h(u)=-\infty$. Thus for $\frac{p M K}{N} \ll u \ll K^{\frac{\epsilon}{4}}$,

$$
f(u) \gg \min \left\{\lim _{u \rightarrow 0^{+}} f(u), f\left(K^{\frac{\epsilon}{4}}\right)\right\} \geq K^{-\frac{\epsilon}{4}} .
$$

Hence,

$$
\omega_{x}^{\prime}(2 r)^{-1} \ll \frac{x}{r} K^{\frac{\epsilon}{4}}
$$


and by induction,

$$
\omega_{x}^{(s)}(2 r) \ll r^{-s+1} .
$$

Recall also that $h_{m, x}^{(l)}(r) \ll G^{-l} K^{\frac{1}{2}-m} B^{l}$, we are done after applying integration by parts repeatedly to the oscillatory integral $\int_{-\infty}^{\infty} h_{m, x}(r) e^{i \omega_{x}(2 r)} d r$

The proof of the Theorem is completed after applying the above Lemma and Weil's bound of the Kloosterman sum to the off-diagonal term. 


\section{CHAPTER 3}

\section{THE SECOND MOMENT OF THE CENTRAL VALUES OF THE SYMMETRIC SQUARE $L$-FUNCTIONS}

In this chapter, we prove Theorem 1.2.5. Since we assume the level 1 throughout, we write $H_{k}$ instead of $H_{k, 1}$. The proof is an adaptation of the proof of the main Theorem in [24]. In their proof they have to consider shifted convolution sum of the Fourier coefficients of Hecke forms. In particular, in the unshifted case, they have to consider a sum of the form

$$
\sum_{K \leq k \leq K+G} \sum_{f \in H_{k}} \sum_{r_{1}, r_{2} \sim K} \frac{a_{f}\left(r_{1}^{2}\right) a_{f}\left(r_{2}^{2}\right)}{\sqrt{r_{1} r_{2}}} .
$$

The fact that they can restrict the sum to those $r_{1}, r_{2}$ of size $K$ (i.e. $r_{1}, r_{2} \sim K$ ) is due to the presence of a compactly supported function coming from the incomplete Poincare series. In our case such feature does not exist, hence we have to consider

$$
\sum_{K \leq k \leq K+G} \sum_{f \in H_{k}} \sum_{r_{1}, r_{2} \ll K^{1+\epsilon}} \frac{a_{f}\left(r_{1}^{2}\right) a_{f}\left(r_{2}^{2}\right)}{\sqrt{r_{1} r_{2}}} .
$$

To overcome the difficulty thus presented, we apply a large sieve type inequality (see Lemma 3.1.4) so that we can consider instead

$$
\sum_{K \leq k \leq K+G} \sum_{f \in H_{k}} \sum_{K^{\frac{2}{3}+\epsilon} \ll r_{1}, r_{2} \ll K^{1+\epsilon}} \frac{a_{f}\left(r_{1}^{2}\right) a_{f}\left(r_{2}^{2}\right)}{\sqrt{r_{1} r_{2}}} .
$$

which turns out to be good enough for the adoption of the proof of Luo and Sarnak and the result obtained here is in agreement with theirs, i.e. we prove that the theorem is true when we take $G \geq K^{\frac{1}{3}}$. 
In the first section we shorten the range of summation of $L\left(\frac{1}{2}, \operatorname{sym}^{2} f\right)$ using an approximate functional equation and a large sieve type inequality for holomorphic cusp forms. In the second and third section we follow P.887-889 of [24] to arrive at our main result.

\subsection{Shortening the length of summation}

We start with the approximate functional equation.

Lemma 3.1.1. For $f \in H_{k}$,

$$
L\left(\frac{1}{2}, \operatorname{sym}^{2} f\right)=2 \sum_{n \ll k^{1+\epsilon}} \frac{a_{f}\left(n^{2}\right)}{\sqrt{n}} \tilde{V}_{k}(n)+O_{\epsilon}\left(k^{-\frac{1}{2}+\epsilon}\right)
$$

where

$$
\tilde{V}_{k}(n)=\frac{1}{2 \pi i} \int_{\operatorname{Re}} G(y) \frac{\zeta(1+2 y)}{y}\left(\frac{k}{n}\right)^{y} d y
$$

for any $A>0$ with

$$
G(y)=\frac{\pi^{-\frac{3}{2} y} 2^{-y} \Gamma\left(\frac{y}{2}+\frac{3}{4}\right)}{\Gamma\left(\frac{3}{4}\right)}
$$

decays exponentially on vertical lines.

Proof. By Lemma 2.2 of [16],

$$
L\left(\frac{1}{2}, \operatorname{sym}^{2} f\right)=2 \sum_{n=1}^{\infty} \frac{a_{f}\left(n^{2}\right)}{\sqrt{n}} V_{k}(n)
$$

where

$$
\begin{aligned}
& V_{k}(x)=\frac{1}{2 \pi i} \int_{\operatorname{Re}} \frac{L_{\infty}\left(\frac{1}{2}+y\right)}{L_{\infty}\left(\frac{1}{2}\right)} \zeta(1+2 y) x^{-y} \frac{d y}{y} \\
& V_{k}(n) \ll_{A}\left(\frac{k}{n}\right)^{A} \text { for any } A>0 .
\end{aligned}
$$


By Corollary 2.3 of [5],

$$
L\left(\frac{1}{2}, \operatorname{sym}^{2} f\right)=2 \sum_{n \leq k^{1+\epsilon}} \frac{a_{f}\left(n^{2}\right)}{\sqrt{n}} V_{k}(n)+O_{\epsilon, A}\left(k^{-A}\right)
$$

for all $A>0$.

By $(2.7)$ in [16],

$$
\begin{gathered}
L\left(\frac{1}{2}, \operatorname{sym}^{2} f\right)=2 \sum_{n \leq k^{1+\epsilon}} \frac{a_{f}\left(n^{2}\right)}{\sqrt{n}} \tilde{V}_{k}(n) \\
+O\left(\sum_{n \leq k^{1+\epsilon}} \frac{\left|a_{f}\left(n^{2}\right)\right|}{\sqrt{n}} k^{-1}\left(\frac{k}{n}\right)^{\epsilon}\right)+O_{\epsilon, A}\left(k^{-A}\right)
\end{gathered}
$$

for all $A>0$. Using Deligne's bound,

$$
L\left(\frac{1}{2}, \operatorname{sym}^{2} f\right)=2 \sum_{n \leq k^{1+\epsilon}} \frac{a_{f}\left(n^{2}\right)}{\sqrt{n}} \tilde{V}_{k}(n)+O_{\epsilon}\left(k^{-\frac{1}{2}+2 \epsilon}\right) .
$$

Since $K$ is large, fro each $K \leq k \leq 3 K$ we have

$$
L\left(\frac{1}{2}, \operatorname{sym}^{2} f\right)=2 \sum_{n \leq K^{1+\epsilon}} \frac{a_{f}\left(n^{2}\right)}{\sqrt{n}} \tilde{V}_{k}(n)+O_{\epsilon}\left(K^{-\frac{1}{2}+2 \epsilon}\right) .
$$

The weight function $\tilde{V}_{t}$ and its derivatives behave nicely with respect to $t$. More precisely,

\section{Lemma 3.1.2.}

$$
\frac{d^{a}}{d t^{a}} \tilde{V}_{t}(n) \ll \frac{1}{t^{a}}\left(\frac{t}{n}\right)^{A}
$$

for all $a \geq 0, A>0$.

Proof. For any $a \geq 0, A>0, \frac{d^{a}}{d t^{a}} \tilde{V}_{t}(n)=$

$$
\begin{aligned}
& \frac{1}{2 \pi i} \frac{1}{t^{a}} \int_{(A)} G(y) \zeta(1+2 y) G(y)(y-1) \cdots(y-a+1)\left(\frac{t}{n}\right)^{y} d y \\
\ll & \frac{1}{t^{a}}\left(\frac{t}{n}\right)^{A}
\end{aligned}
$$


We can now restrict the sum over $n$ to those $n$ less than $K^{1+\epsilon}$.

\section{Lemma 3.1.3.}

$$
\begin{aligned}
& \sum_{k \text { even }} h\left(\frac{k-K-1}{G}\right) \sum_{f \in H_{k}} \frac{2 \pi^{2}}{L\left(1, \mathrm{sym}^{2} f\right)} \frac{L^{2}\left(\frac{1}{2}, \mathrm{sym}^{2} f\right)}{k-1} \\
\ll & \sum_{k \text { even }} h\left(\frac{k-K-1}{G}\right) \sum_{f \in H_{k}}\left|\sum_{n \leq K^{1+\epsilon}} \frac{a_{f}\left(n^{2}\right)}{\sqrt{n}} \tilde{V}_{k}(n)\right|^{2} \times \\
& \frac{2 \pi^{2}}{L\left(1, \mathrm{sym}^{2} f\right)(k-1)}+O(G) .
\end{aligned}
$$

Proof. By Lemma 2.1,

$$
\begin{aligned}
& \sum_{k \text { even }} h\left(\frac{k-K-1}{G}\right) \sum_{f \in H_{k}} \frac{2 \pi^{2}}{L\left(1, \mathrm{sym}^{2} f\right)} \frac{L^{2}\left(\frac{1}{2}, \mathrm{sym}^{2} f\right)}{k-1} \\
= & \sum_{k \text { even }} h\left(\frac{k-K-1}{G}\right) \sum_{f \in H_{k}} \frac{2 \pi^{2}}{L\left(1, \mathrm{sym}^{2} f\right)(k-1)} \times \\
& \left|\sum_{n \ll K^{1+\epsilon}} \frac{a_{f}\left(n^{2}\right)}{\sqrt{n}} \tilde{V}_{k}(n)+O_{\epsilon}\left(k^{-\frac{1}{2}+\epsilon}\right)\right|^{2} .
\end{aligned}
$$

Since $|a+b|^{2} \ll|a|^{2}+|b|^{2}$, the error term contributes

$$
O\left(\sum_{k \text { even }} h\left(\frac{k-K-1}{G}\right) \sum_{f \in H_{k}} \frac{K^{-1+2 \epsilon}}{L\left(1, \mathrm{sym}^{2} f\right)(k-1)}\right) .
$$

Using the following bound (see P. 6 of [1]),

$$
k^{-\epsilon} \ll L\left(1, \operatorname{sym}^{2} f\right)
$$

The error term contributes $O(G)$.

The following Large Sieve type inequality will be crucial later, making the adoption of the proof in [24] possible.

Lemma 3.1.4. Notations as before. Let $\theta(t, x)$ be a smooth function such that

$$
\begin{aligned}
\partial_{2} \theta(t, x) & \ll x^{-1} t^{\epsilon} \text { for all } \epsilon>0 \\
\theta(t, x) & \ll x^{\epsilon} \text { for all } \epsilon>0
\end{aligned}
$$


and let $\left\{b_{n}\right\}_{n=N}^{2 N}$ be a set of complex numbers. Then for $1 \leq G \leq K$

$$
\sum_{\substack{K \leq k \leq K+G \\ k \text { even }}} \sum_{f \in H_{k}}\left|\sum_{n=N}^{2 N} a_{f}(n) \theta(k, n) b_{n}\right|^{2} \ll(K N)^{\epsilon}(K G+N) \sum_{n=N}^{2 N}\left|b_{n}\right|^{2} .
$$

Proof. Let $A_{f}(x)=\sum_{n=N}^{x} a_{f}(n) b_{n}$. Then by the Abel summation formula,

$$
\sum_{n=N}^{2 N} a_{f}(n) b_{n} \theta(k, n)=A_{f}(2 N) \theta(k, 2 N)-\int_{N}^{2 N} A_{f}(x) \partial_{2} \theta(k, x) d x .
$$

Hence by Cauchy-Schwartz inequality,

$$
\begin{aligned}
&\left|\sum_{n=N}^{2 N} a_{f}(n) b_{n} \theta(k, n)\right|^{2} \ll\left|A_{f}(2 N)\right|^{2} N^{\epsilon}+\int_{N}^{2 N}\left|A_{f}(x)\right|^{2} d x \int_{N}^{2 N}\left|\partial_{2} \theta(k, x)\right|^{2} d x \\
& \ll\left|A_{f}(2 N)\right|^{2} N^{\epsilon}+N^{\epsilon} \int_{N}^{2 N}\left|A_{f}(x)\right|^{2} d x \int_{N}^{2 N} x^{-2} k^{\epsilon} d x .
\end{aligned}
$$

Using Lemma 8 of [15], we get

$$
\sum_{\substack{K \leq k \leq K+G \\ k \text { even }}} \sum_{f \in H_{k}}\left|\sum_{n=N}^{2 N} a_{f}(n) \theta(k, n) b_{n}\right|^{2} \ll(K N)^{\epsilon}(K G+N) \sum_{n=N}^{2 N}\left|b_{n}\right|^{2} .
$$

Taking $\theta(t, x)=\tilde{V}_{t}(x)$, we get

$$
\begin{gathered}
b_{n}=\left\{\begin{array}{l}
n^{-\frac{1}{4}} \text { if } n \text { is a square } \\
0 \text { otherwise. }
\end{array}\right. \\
\sum_{\substack{K \leq k \leq K+G \\
k \text { even }}} \sum_{f \in H_{k}}\left|\sum_{n \ll K^{\frac{2}{3}+\epsilon}} \frac{a_{f}\left(n^{2}\right) \tilde{V}_{k}(n)}{\sqrt{n}}\right|^{2} \\
=\sum_{\substack{K \leq k \leq K+G \\
k \text { even }}} \sum_{f \in H_{k}}\left|\sum_{n \ll K^{\frac{4}{3}}+2 \epsilon} a_{f}(n) \theta(k, n) b_{n}\right|^{2} .
\end{gathered}
$$


We then apply the large sieve inequality and sum dyadically, for each $N=2^{t}$, $0 \leq t \leq\left(\frac{4}{3}+2 \epsilon\right) \log _{2} K$,

$$
\sum_{\substack{K \leq k \leq K+G \\ k \text { even }}} \sum_{f \in H_{k}}\left|\sum_{n=N}^{2 N} a_{f}(n) \theta(k, n) b_{n}\right|^{2} \ll K^{\epsilon}(K G+N) \sum_{n=N}^{2 N}\left|b_{n}\right|^{2} .
$$

Summing over all these $N$, we get

$$
\sum_{\substack{K \leq k \leq K+G \\ k \text { even }}} \sum_{f \in H_{k}}\left|\sum_{n \ll K^{\frac{2}{3}+\epsilon}} \frac{a_{f}\left(n^{2}\right) \tilde{V}_{k}(n)}{\sqrt{n}}\right|^{2} \ll K^{\epsilon}\left(K G+K^{\frac{4}{3}+2 \epsilon}\right) \ll K^{1+\epsilon} G .
$$

Hence from now on, we can consider instead

$$
\sum_{k \text { even }} h\left(\frac{k-K-1}{G}\right) \sum_{f \in H_{k}} \frac{2 \pi^{2}}{L\left(1, \operatorname{sym}^{2} f\right)(k-1)}\left|\sum_{K^{\frac{2}{3}+\epsilon} \ll n \ll K^{1+\epsilon}} \frac{a_{f}\left(n^{2}\right)}{\sqrt{n}} \tilde{V}_{k}(n)\right|^{2} .
$$

\subsection{Further manipulation}

We then apply Lemma 3.1.4 and sum up dyadically.

For each $N=2^{t}, 0 \leq t \leq\left(\frac{4}{3}+2 \epsilon\right) \log _{2} K$,

$$
\sum_{\substack{K \leq k \leq K+G \\ k \text { even }}} \sum_{f \in H_{k}}\left|\sum_{n=N}^{2 N} a_{f}(n) \theta(k, n) b_{n}\right|^{2} \ll K^{\epsilon}(K G+N) \sum_{n=N}^{2 N}\left|b_{n}\right|^{2} .
$$

Summing over all these $N$, we get

$$
\sum_{\substack{K \leq k \leq K+G \\ k \text { even }}} \sum_{f \in H_{k}}\left|\sum_{n \ll K^{\frac{2}{3}+\epsilon}} \frac{a_{f}\left(n^{2}\right) \tilde{V}_{k}(n)}{\sqrt{n}}\right|^{2} \ll K^{\epsilon}\left(K G+K^{\frac{4}{3}+2 \epsilon}\right) \ll K^{1+\epsilon} G .
$$

Hence from now on, we can consider instead

$$
\begin{aligned}
\sum_{k \text { even }} h\left(\frac{k-K-1}{G}\right) & \sum_{f \in H_{k}} \frac{2 \pi^{2}}{L\left(1, \operatorname{sym}^{2} f\right)(k-1)} \times \\
& \left|\sum_{K^{\frac{2}{3}+\epsilon} \ll n \ll K^{1+\epsilon}} \frac{a_{f}\left(n^{2}\right)}{\sqrt{n}} \tilde{V}_{k}(n)\right|^{2} .
\end{aligned}
$$


We now perform a smooth dyadic argument. Let $\eta$ be a $C^{\infty}$ function such that

$$
\eta(x)=\left\{\begin{array}{l}
1 \text { if } x \in(1,2] \\
0 \text { if } x \notin\left(\frac{1}{2}, 3\right)
\end{array}\right.
$$

$S(x)=\sum_{j=0}^{\infty} \eta\left(\frac{x}{2^{j}}\right) ;$ and let for each $0 \leq j, g_{2^{j}}(x)=\frac{\eta\left(\frac{x}{2^{j}}\right)}{S(x)}$. Then for any $x \geq 1$ $\sum_{j=0}^{\infty} g_{2^{j}}(x)=1$. Thus $(3.2 .1)=$

$$
\begin{aligned}
& \sum_{k \text { even }} h\left(\frac{k-K-1}{G}\right) \sum_{f \in H_{k}} \frac{2 \pi^{2}}{L\left(1, \operatorname{sym}^{2} f\right)(k-1)} \times \\
& \left|\sum_{j=0}^{\infty} \sum_{K^{\frac{2}{3}+\epsilon} \ll n \ll K^{1+\epsilon}} g_{2^{j}}(n) \frac{a_{f}\left(n^{2}\right)}{\sqrt{n}} \tilde{V}_{k}(n)\right|^{2}
\end{aligned}
$$

Since for $K^{\frac{2}{3}+\epsilon} \ll n \ll K^{1+\epsilon}, g_{2^{j}}(n)=0$ whenever $j \geq(1+2 \epsilon) \log _{2} K$ or $j \leq$ $\left(\frac{2}{3}-\epsilon\right) \log _{2} K$. Let $v_{1}=\left(\frac{2}{3}-\epsilon\right) \frac{\log K}{\log 2}, v_{2}=(1+2 \epsilon) \frac{\log K}{\log 2}-1$, then (3.2.2)

$$
\begin{aligned}
\leq & \sum_{k \text { even }} h\left(\frac{k-K-1}{G}\right) \sum_{f \in H_{k}} \frac{2 \pi^{2}}{L\left(1, \mathrm{sym}^{2} f\right)(k-1)} \times \\
& \sum_{t=v_{1}}^{v_{2}}\left|\sum_{n=1}^{\infty} g_{2^{t}}(n) \frac{a_{f}\left(n^{2}\right)}{\sqrt{n}} \tilde{V}_{k}(n)\right|^{2}\left(\sum_{t=v_{1}}^{v_{2}} 1\right) \\
\ll & K^{\epsilon} \sum_{k \text { even }} h\left(\frac{k-K-1}{G}\right) \sum_{f \in H_{k}} \frac{2 \pi^{2}}{L\left(1, \mathrm{sym}^{2} f\right)(k-1)} \times \\
& \sum_{t=v_{1}}^{v_{2}}\left|\sum_{n=1}^{\infty} g_{2^{t}}(n) \frac{a_{f}\left(n^{2}\right)}{\sqrt{n}} \tilde{V}_{k}(n)\right|^{2} .
\end{aligned}
$$

We will show for each $T=2^{t}, v_{1} \leq t \leq v_{2}$,

$$
\begin{aligned}
& K^{\epsilon} \sum_{k \text { even }} h\left(\frac{k-K-1}{G}\right) \sum_{f \in H_{k}} \frac{2 \pi^{2}}{L\left(1, \mathrm{sym}^{2} f\right)(k-1)} \times \\
& \left|\sum_{n \geq 1} \frac{g_{T}(n) a_{f}\left(n^{2}\right) \tilde{V}_{k}(n)}{\sqrt{n}}\right|^{2} \ll \frac{T^{2} K^{\epsilon}}{K G^{2}} .
\end{aligned}
$$


By Petersson formula, the above sum

$$
\begin{aligned}
= & K^{\epsilon} \sum_{k \text { even }} h\left(\frac{k-K-1}{G}\right) \sum_{n_{1}, n_{2}} \frac{g_{T}\left(n_{1}\right) g_{T}\left(n_{2}\right) \tilde{V}_{k}\left(n_{1}\right) \tilde{V}_{k}\left(n_{2}\right)}{\sqrt{n_{1} n_{2}}} \times \\
& {\left[\delta_{n_{1}, n_{2}}+2 \pi i^{-k} \sum_{c=1}^{\infty} \frac{S\left(n_{1}^{2}, n_{2}^{2} ; c\right)}{c} J_{k-1}\left(\frac{4 \pi n_{1} n_{2}}{c}\right)\right] . }
\end{aligned}
$$

By $(5.102)$ in $[19], J_{k-1}(x) \ll\left(\frac{e x}{2 k}\right)^{k-1}$, so the contribution from those $c>\frac{24 \pi n_{1} n_{2}}{K}$ is exponentially small. Thus it suffices to consider those $c$ less than or equal to $\frac{24 \pi n_{1} n_{2}}{K}$, i.e.

$$
\begin{gathered}
K^{\epsilon} \sum_{k \text { even }} h\left(\frac{k-K-1}{G}\right) \sum_{n_{1}, n_{2}} \frac{g_{T}\left(n_{1}\right) g_{T}\left(n_{2}\right) \tilde{V}_{k}\left(n_{1}\right) \tilde{V}_{k}\left(n_{2}\right)}{\sqrt{n_{1} n_{2}}} \\
\times \quad\left[\delta_{n_{1} n_{2}}+2 \pi i^{-k} \sum_{c \leq \frac{24 \pi n_{1} n_{2}}{K}} \frac{S\left(n_{1}^{2}, n_{2}^{2} ; c\right)}{c} J_{k-1}\left(\frac{4 \pi n_{1} n_{2}}{c}\right)\right] .
\end{gathered}
$$

Note that the diagonal contribution is $\ll K^{2 \epsilon} G$.

\subsection{The off-diagonal contribution}

Thus, it remains to treat the off-diagonal term, that is $K^{\epsilon}$ times

$$
\begin{aligned}
& \sum_{n_{1}, n_{2}} \frac{g_{T}\left(n_{1}\right) g_{T}\left(n_{2}\right)}{\sqrt{n_{1} n_{2}}} \sum_{c \leq \frac{24 \pi n_{1} n_{2}}{K}} \frac{S\left(n_{1}^{2}, n_{2}^{2} ; c\right)}{c} \times \\
& \quad \sum_{k \text { even }} i^{-k} h\left(\frac{k-K-1}{G}\right) \tilde{V}_{k}\left(n_{1}\right) \tilde{V}_{k}\left(n_{2}\right) J_{k-1}\left(\frac{4 \pi n_{1} n_{2}}{c}\right) .
\end{aligned}
$$

\subsubsection{Restricting the sum over $c$}

We now show that we can further restrict the sum over $c$ to those $c \ll \frac{n_{1} n_{2} K^{\epsilon / 2}}{K G}$. The proof is essentially the same as on P.880-882 of [24]. 
Let $f(t):=f_{n_{1}, n_{2}}(t)=h\left(\frac{t-K}{G}\right) \tilde{V}_{t}\left(n_{1}\right) \tilde{V}_{t}\left(n_{2}\right)$.

By Lemma 4.1 in [24],

$$
\begin{aligned}
& \sum_{k \text { even }} i^{-k} h\left(\frac{k-K-1}{G}\right) \tilde{V}_{t}\left(n_{1}\right) \tilde{V}_{t}\left(n_{2}\right) J_{k-1}\left(\frac{4 \pi n_{1} n_{2}}{c}\right) \\
= & \int_{-\infty}^{\infty} \hat{f}(t) \sin \left(\frac{4 \pi n_{1} n_{2}}{c} \cos 2 \pi t\right) d t .
\end{aligned}
$$

When $c \gg \frac{n_{1} n_{2} K^{\epsilon / 2}}{K G}, \frac{4 \pi n_{1} n_{2}}{c} G^{-4} \ll K^{1-\epsilon / 2} G^{-3} \ll K^{-\epsilon / 2}$.

Using (4.4), (4.5), Lemma 4.2 in $[24]$ and in view of the fact that $\int_{-\infty}^{\infty}\left|\hat{f}(t) t^{r}\right| d t \ll$ $G^{-r}$ (since $\frac{d^{r}}{d t^{r}} h\left(\frac{t-K}{G}\right) \ll G^{-r}$ and see also Lemma 3$)$, the sum

$$
\begin{aligned}
& \sum_{n_{1}, n_{2}} \frac{g_{T}\left(n_{1}\right) g_{T}\left(n_{2}\right)}{\sqrt{n_{1} n_{2}}} \sum_{c \gg \frac{n_{1} n_{2} K^{\epsilon / 2}}{K G}} \frac{S\left(n_{1}^{2}, n_{2}^{2} ; c\right)}{c} \times \\
& \int_{-\infty}^{\infty} \hat{f}(t) \sin \left(\frac{4 \pi n_{1} n_{2}}{c} \cos 2 \pi t\right) d t \\
= & \sum_{n_{1}, n_{2}} \frac{g_{T}\left(n_{1}\right) g_{T}\left(n_{2}\right)}{\sqrt{n_{1} n_{2}}} \mid \sum_{c \gg \frac{n_{1} n_{2} K^{\epsilon / 2}}{K G}} \frac{S\left(n_{1}^{2}, n_{2}^{2} ; c\right)}{c} \times \\
& \int_{0}^{\infty} f^{(p)}\left(\sqrt{\frac{8 \pi n_{1} n_{2} y}{c}}\right) \sin \left(y+\frac{4 \pi n_{1} n_{2}}{c}-\frac{\pi}{4}\right) \frac{d y}{\sqrt{y}} \mid+O\left(K^{-A}\right)
\end{aligned}
$$

for all $A>0$ upon taking $p>\left\lfloor\frac{2 A+200}{\epsilon}\right\rfloor+1$. Note that

$$
f^{(p)}\left(\sqrt{\frac{8 \pi n_{1} n_{2} y}{c}}\right)=h^{(p)}\left(\frac{\sqrt{\frac{8 \pi n_{1} n_{2}}{c} y}-K}{G}\right),
$$

hence $\frac{1}{\sqrt{y}} \sim \sqrt{\frac{8 \pi n_{1} n_{2}}{c}} \frac{1}{K}$, and

$$
\begin{aligned}
& \frac{d^{j}}{d y^{j}} f^{(p)}\left(\sqrt{\frac{8 \pi n_{1} n_{2} y}{c}}\right) \\
\ll & \max \left\{\left(\frac{1}{G} \sqrt{\frac{8 \pi n_{1} n_{2}}{c y}}\right)^{j}, \cdots, y^{-j+1}\left(\frac{1}{G} \sqrt{\frac{8 \pi n_{1} n_{2}}{c y}}\right)\right\} .
\end{aligned}
$$




$$
\begin{aligned}
& \text { If } \frac{n_{1} n_{2}}{c} \ll \frac{K G}{K^{\frac{\epsilon}{2}}} \text { then we get } \\
& \qquad \frac{1}{G} \sqrt{\frac{8 \pi n_{1} n_{2}}{c y}} \ll K^{-\frac{\epsilon}{2}}
\end{aligned}
$$

and

$$
y^{-1} \ll \frac{G}{K} K^{-\frac{\epsilon}{2}}
$$

Hence if $\frac{n_{1} n_{2}}{c} \ll \frac{K G}{K^{\frac{\epsilon}{2}}}$, by applying partial integration sufficiently many times, we see that

$$
\int_{0}^{\infty} f^{(p)}\left(\sqrt{\frac{8 \pi n_{1} n_{2} y}{c}}\right) \sin \left(y+\frac{4 \pi n_{1} n_{2}}{c}-\frac{\pi}{4}\right) \frac{d y}{\sqrt{y}}
$$

is negligible. Hence the total contribution is small. We can now concentrate on

$$
\sum_{n_{1}, n_{2}} \frac{g_{T}\left(n_{1}\right) g_{T}\left(n_{2}\right)}{\sqrt{n_{1} n_{2}}} \sum_{c \ll \frac{n_{1} n_{2} K^{\epsilon} / 2}{K G}} \frac{S\left(n_{1}^{2}, n_{2}^{2} ; c\right)}{c} \int_{-\infty}^{\infty} \hat{f}(t) \sin \left(\frac{4 \pi n_{1} n_{2}}{c} \cos 2 \pi t\right) d t .
$$

\subsubsection{Applying the stationary phase lemma}

As in P.882 of [24], we can consider instead

$$
\begin{aligned}
& \max _{p_{1}+p_{2}+p_{3} \leq 14 N}\left\{\mid \sum_{n_{1}, n_{2}} \frac{g_{T}\left(n_{1}\right) g_{T}\left(n_{2}\right)}{\sqrt{n_{1} n_{2}}} \sum_{c \ll \frac{n_{1} n_{2} K^{\epsilon / 2}}{K G}} \frac{S\left(n_{1}^{2}, n_{2}^{2} ; c\right)}{c} e\left(\frac{2 n_{1} n_{2}}{c}\right)\right. \\
& \times \int_{0}^{\infty} \frac{\partial^{p_{1}} h}{\partial y^{p_{1}}}\left(\frac{y-K-1}{G}\right) \frac{\partial^{p_{2}} \tilde{V}_{y}}{\partial y^{p_{2}}}\left(n_{1}\right) \frac{\partial^{p_{3}} \tilde{V}_{y}}{\partial y^{p_{3}}}\left(n_{2}\right) \times \\
& \left.\int_{-\infty}^{\infty} e\left(\frac{2 n_{1} n_{2}}{c}\left(-2 \pi^{2} t^{2}+\frac{2}{3} \pi^{4} t^{4}\right)+t y\right) d t d y \mid\right\} .
\end{aligned}
$$

We now apply the principle of stationary phase to evaluate asymptotically the integral

$$
\int_{-\infty}^{\infty} e\left(\frac{2 n_{1} n_{2}}{c}\left(-2 \pi^{2} t^{2}+\frac{2}{3} \pi^{4} t^{4}\right)+t y\right) d t
$$

as in P.883-884 of [24]. 
Let $f(t)=\frac{4 \pi n_{1} n_{2}}{c}\left(-2 \pi^{2} t^{2}+\frac{2}{3} \pi^{4} t^{4}\right)+2 \pi t y$. Then

$$
f^{\prime}(t)=\frac{4 \pi n_{1} n_{2}}{c}\left(-4 \pi^{2} t+\frac{8}{3} \pi^{4} t^{3}\right)+2 \pi y
$$

and

$$
f^{\prime \prime}(t)=\frac{4 \pi n_{1} n_{2}}{c}\left(-4 \pi^{2}+8 \pi^{4} t^{2}\right)
$$

Since $-4 \pi^{2} t+\frac{8}{3} \pi^{4} t^{3}$ has the roots $0, \sqrt{\frac{3}{2}} \pi^{-1}$ and $-\sqrt{\frac{3}{2}} \pi^{-1}$ and $\frac{y c}{4 \pi n_{1} n_{2}} \sim \frac{K c}{n_{1} n_{2}} \ll$ $\frac{K^{\epsilon / 2}}{G}$, the integral has stationary phases at $t_{1}, \sqrt{\frac{3}{2}} \pi^{-1}-t_{2},-\sqrt{\frac{3}{2}} \pi^{-1}-t_{3}$ with $t_{i}=t_{i}\left(y, \frac{4 \pi n_{1} n_{2}}{c}\right) \sim \frac{y c}{4 \pi n_{1} n_{2}} \ll \frac{K^{\epsilon / 2}}{G}$ for $i=1,2,3$ by the mean value theorem.

Let $\beta$ be any critical point as above. Then $\beta$ satisfies $\beta_{y}=\frac{c}{8 \pi^{2}\left(1-2 \pi^{2} \beta^{2}\right)}$,

$$
\beta_{n_{i}}=\frac{-y c}{8 \pi^{2} n_{1} n_{2} n_{i}\left(1-2 \pi^{2} \beta^{2}\right)} \text { and }\left|\beta_{n_{i}}\right| \sim \frac{c K}{n_{1} n_{2} n_{i}} .
$$

We have the same asymptotic expression for our oscillatory integral as in P.884 of [24], where their $x$ is our $\frac{4 \pi n_{1} n_{2}}{c}$. To see that the $O\left(\frac{c}{n_{1} n_{2}}\right)$ term contributes $O\left(G K^{\epsilon}\right)$ one notices that Weil's bound gives $S\left(n_{1}^{2}, n_{2}^{2} ; c\right) \ll_{\epsilon}\left(n_{1}^{2}, n_{2}^{2}, c\right)^{\frac{1}{2}} c^{\frac{1}{2}+\epsilon}$. Hence the contribution of the $O\left(\frac{c}{n_{1} n_{2}}\right)$ term is

$$
\begin{aligned}
& \ll G \sum_{n_{1}, n_{2}} \frac{g_{T}\left(n_{1}\right) g_{T}\left(n_{2}\right)}{\sqrt{n_{1} n_{2}}} \sum_{c \ll \frac{n_{1} n_{2} K^{\epsilon / 2}}{K G}} \frac{\left(n_{1}^{2}, n_{2}^{2}, c\right)^{\frac{1}{2}} c^{\frac{1}{2}+\epsilon}}{n_{1} n_{2}} \\
& \ll G \frac{1}{T^{3}} \sum_{T \leq n_{2}<2 T} \sum_{c \ll \frac{T^{2} K^{\epsilon / 2}}{K G}} \sum_{T \leq n_{1}<2 T}\left(n_{1}^{2}, c\right)^{\frac{1}{2}} c^{\frac{1}{2}+\epsilon} \\
& \ll \frac{G}{T} \sum_{c \ll \frac{T^{2} K^{\epsilon / 2}}{K G}} c^{\frac{1}{2}+\epsilon} \ll G \frac{1}{T}\left(\frac{T^{2} K^{\epsilon / 2}}{K G}\right)^{\frac{3}{2}+\epsilon} \ll G \frac{K^{\epsilon} T^{2}}{K^{\frac{3}{2}} G^{\frac{3}{2}}} .
\end{aligned}
$$

As stated in P.884 of [24], we only have to consider

$$
\max _{p_{1}+p_{2}+p_{3}=p \leq 14 N}\left\{\mid \sum_{n_{1}, n_{2}} \frac{g_{T}\left(n_{1}\right) g_{T}\left(n_{2}\right)}{\sqrt{n_{1} n_{2}}} \sum_{c \ll \frac{n_{1} n_{2} K^{\epsilon / 2}}{K G}} \frac{S\left(n_{1}^{2}, n_{2}^{2} ; c\right)}{c}\right.
$$




$$
\begin{aligned}
& \quad \times \quad e\left(\frac{2 n_{1} n_{2}}{c}\right) \int_{0}^{\infty} \frac{\partial^{p_{1}} h}{\partial y^{p_{1}}}\left(\frac{y-K-1}{G}\right) \frac{\partial^{p_{2}} \tilde{V}_{y}}{\partial y^{p_{2}}}\left(n_{1}\right) \frac{\partial^{p_{3}} \tilde{V}_{y}}{\partial y^{p_{3}}}\left(n_{2}\right) \sqrt{\frac{c}{n_{1} n_{2}}} \\
& \left.\quad \times \quad\left|-4 \pi^{2}+8 \pi^{4} t_{1}^{4}\right|^{-\frac{1}{2}} e\left(\frac{2 n_{1} n_{2}}{c}\left(-2 \pi^{2} t_{1}^{2}+\frac{2 \pi^{4}}{3} t_{1}^{4}\right)+t_{1} y\right) d y \mid\right\} . \\
& \text { Let } F_{c}\left(x_{1}, x_{2}\right)=\int_{0}^{\infty} h^{\left(p_{1}\right)}(y) \partial_{1}^{p_{2}} \tilde{V}\left(G y+K+1, x_{1}\right) \partial_{1}^{p_{3}} \tilde{V}\left(G y+K+1, x_{2}\right) \\
& \quad \times \quad\left|-4 \pi^{2}+8 \pi^{4} t_{1}^{4}\right|^{-\frac{1}{2}} \\
& \quad \times \quad e\left(\frac{2 x_{1} x_{2}}{c}\left(-2 \pi^{2} t_{1}^{2}+\frac{2 \pi^{4}}{3} t_{1}^{4}\right)+G t_{1} y+(K+1) t_{1}\right) \frac{d y}{x_{1} x_{2}}
\end{aligned}
$$

with the convention that $\tilde{V}_{t}(x)=\tilde{V}(t, x)$ and $\partial_{i}$ means differentiating with respect to the $i$ th coordinate. Note that we are suppressing the dependence in $p_{1}, p_{2}$ and $p_{3}$ as it won't fundamentally change the analysis.

A typical sum in (3.3.3) is (after a change of variable)

$$
G \sum_{n_{1}, n_{2}} g_{T}\left(n_{1}\right) g_{T}\left(n_{2}\right) \sum_{c \ll \frac{n_{1} n_{2} K^{\epsilon} / 2}{K G}} \frac{S\left(n_{1}^{2}, n_{2}^{2} ; c\right)}{\sqrt{c}} e\left(\frac{2 n_{1} n_{2}}{c}\right) F_{c}\left(n_{1}, n_{2}\right) .
$$

\subsubsection{Applying the Poisson summation formula}

As in [24], we will separate the sum over $n_{1}, n_{2}$ into congruence classes modulo $c$ and then apply the Poisson summation formula. To see that all but the $(0,0)$-Fourier transform are insignificant, we need the following lemma.

Lemma 3.3.1. For $K^{\frac{2}{3}+\epsilon} \ll x_{1}, x_{2} \ll K^{1+\epsilon}, \frac{x_{1} x_{2}}{c} \gg \frac{K G}{K^{\epsilon / 2}}$,

$$
\frac{\partial^{m_{1}+m_{2}}}{\partial x_{1}^{m_{1}} \partial x_{2}^{m_{2}}} F_{c}\left(x_{1}, x_{2}\right) \ll K^{-\frac{\epsilon\left(m_{1}+m_{2}\right)}{2}}
$$

for all $m_{1}, m_{2}>0$.

Remark 3.3.2. The fact that we can assume $x_{1}, x_{2} \gg K^{\frac{2}{3}+\epsilon}$ is crucial here. This is the main point of departure from [24] in which they have $x_{1}, x_{2} \sim K$. 
Proof. (of Lemma 3.3.1) From

$$
\tilde{V}(t, u)=\frac{1}{2 \pi i} \int G(y) \frac{\zeta(1+2 y)}{y}\left(\frac{t}{u}\right)^{y} d y,
$$

it is easy to see that $\partial_{1}^{a} \partial_{2}^{b} \tilde{V}(t, u) \ll \frac{1}{t^{a}} \frac{1}{u^{b}}\left(\frac{t}{u}\right)^{A}$ for all $a>0, b>0$ and $A>0$. We see from (3.3.2) that

$$
\begin{aligned}
& \frac{\partial^{m_{1}+m_{2}}}{\partial x_{1}^{m_{1}} \partial x_{2}^{m_{2}}} F_{c}\left(x_{1}, x_{2}\right) \ll\left(K \frac{\partial t_{1}}{\partial x_{1}}\right)^{m_{1}}\left(K \frac{\partial t_{1}}{\partial x_{2}}\right)^{m_{2}} \\
+ & \left(\frac{x_{1} x_{2}}{c} t_{1} \frac{\partial t_{1}}{\partial x_{1}}\right)^{m_{1}}\left(\frac{x_{1} x_{2}}{c} t_{1} \frac{\partial t_{1}}{\partial x_{2}}\right)^{m_{2}} \\
\ll & \left(\frac{K^{2} c}{x_{1}^{2} x_{2}}\right)^{m_{1}}\left(\frac{K^{2} c}{x_{1} x_{2}^{2}}\right)^{m_{2}} \ll K^{-\frac{\epsilon\left(m_{1}+m_{2}\right)}{2}} .
\end{aligned}
$$

Then we can consider instead

$$
G \sum_{n_{1}, n_{2}} g_{T}\left(n_{1}\right) g_{T}\left(n_{2}\right) \sum_{c \ll \frac{n_{1} n_{2} K^{\epsilon / 2}}{K G}} \frac{S\left(n_{1}^{2}, n_{2}^{2} ; c\right)}{\sqrt{c}} e\left(\frac{2 n_{1} n_{2}}{c}\right) F_{c}\left(n_{1}, n_{2}\right) .
$$

Since supp $g_{T} \subset(T / 2,3 T)$, (3.3.5) can be replaced by

$$
\begin{aligned}
& G \sum_{\substack{c \ll \frac{T^{2} K^{\epsilon} / 2}{K G}\\
}} \frac{1}{\sqrt{c}} \sum_{a, b(c)} S\left(a^{2}, b^{2} ; c\right) e\left(\frac{2 a b}{c}\right) \\
\times & \sum_{\substack{n_{1} \equiv a(c) \\
n_{2} \equiv b(c)}} F_{c}\left(n_{1}, n_{2}\right) g_{T}\left(n_{1}\right) g_{T}\left(n_{2}\right) \\
= & G \sum_{\substack{c \ll \frac{T^{2} K^{\epsilon} / 2}{K G}\\
}} c^{-\frac{5}{2}} \sum_{a, b(c)} \sum_{u, v(c)} S\left(a^{2}, b^{2} ; c\right) e\left(\frac{2 a b+u a+v b}{c}\right) \times \\
& \sum_{n_{1}, n_{2}} F_{c}\left(n_{1}, n_{2}\right) g_{T}\left(n_{1}\right) g_{T}\left(n_{2}\right) e\left(-\frac{u}{c} n_{1}\right) e\left(-\frac{v}{c} n_{2}\right) .
\end{aligned}
$$

By the Poisson summation formula, (3.3.5) can be replaced by

$$
G \sum_{c \ll \frac{T^{2} K^{\epsilon / 2}}{K G}} c^{-\frac{5}{2}} \sum_{a, b(c)} \sum_{u, v(c)} S\left(a^{2}, b^{2} ; c\right) e\left(\frac{2 a b+u a+v b}{c}\right) \times
$$




$$
\sum_{m_{1}, m_{2}} \iint_{\mathbb{R}^{2}} F_{c}\left(x_{1}, x_{2}\right) e\left(\left(-\frac{u}{c}+m_{1}\right) x_{1}\right) e\left(\left(-\frac{v}{c}+m_{2}\right) x_{2}\right) d x_{1} d x_{2} .
$$

\subsubsection{Application of the two dimensional Van der Corput lemma}

Picking $|u|,|v| \leq \frac{c}{2}$ and integrating by parts sufficiently many times (using Lemma 3.3.1), (3.3.5) can be replaced by

$$
\begin{aligned}
& G \sum_{c \ll \frac{T^{2} K^{\epsilon / 2}}{K G}} c^{-\frac{5}{2}} \sum_{a, b(c)} \sum_{u, v(c)} S\left(a^{2}, b^{2} ; c\right) e\left(\frac{2 a b+u a+v b}{c}\right) \\
& \times \quad \iint_{R_{T}} F_{c}\left(x_{1}, x_{2}\right) e\left(\frac{-u x_{1}-v x_{2}}{c}\right) d x_{1} d x_{2}
\end{aligned}
$$

where $R_{T}$ is the rectangle in which $x_{1} \sim T$ and $x_{2} \sim T$.

Let

$$
\phi_{u, v, c}\left(x_{1}, x_{2}, z\right)=\frac{2 x_{1} x_{2}}{c}\left(-2 \pi^{2} t_{1}^{2}+\frac{2 \pi^{4}}{3} t_{1}^{4}\right)+G z t_{1}+(K+1) t_{1}
$$

where $t_{1}$ depends on both $x_{1}, x_{2}$ and $z$ (see Section 3.3.2).

Then, (3.3.6)

$$
\begin{aligned}
= & G \sum_{c \ll \frac{T^{2} K^{\epsilon} / 2}{K G}} c^{-\frac{5}{2}} \sum_{a, b(c)} \sum_{u, v(c)} S\left(a^{2}, b^{2} ; c\right) e\left(\frac{2 a b+u a+v b}{c}\right) \times \\
& \int_{0}^{\infty} \iint_{R_{T}} h^{\left(p_{1}\right)}(z) \partial_{1}^{p_{2}} \tilde{V}\left(G z+K-1, x_{1}\right) \partial_{1}^{p_{3}} \tilde{V}\left(G z+K-1, x_{2}\right) \times \\
& \left|4 \pi^{2}-8 \pi^{4} t_{1}^{2}\right|^{-\frac{1}{2}} e\left(\phi_{u, v, c}\left(x_{1}, x_{2}, z\right)\right) e\left(\frac{-u x_{1}-v x_{2}}{c}\right) \frac{d x_{1} d x_{2}}{x_{1} x_{2}} d z
\end{aligned}
$$

Differentiating $\phi_{u, v, c}$ (which we write as $\phi$ for brevity),

$$
\begin{aligned}
\phi_{x_{1}} & =\frac{2 x_{2}}{c}\left(-2 \pi^{2} t_{1}^{2}+\frac{2 \pi^{4}}{3} t_{1}^{4}\right)+\frac{2 x_{1} x_{2}}{c}\left(-4 \pi^{2} t_{1} \frac{\partial t_{1}}{\partial x_{1}}+\frac{8 \pi^{4}}{3} t_{1}^{3} \frac{\partial t_{1}}{\partial x_{1}}\right) \\
& +(G z+K+1) \frac{\partial t_{1}}{\partial x_{1}} .
\end{aligned}
$$


By (2.5)

$$
\frac{\partial t_{1}}{\partial x_{1}}=\frac{c(G z+K+1)}{8 \pi^{2} x_{1}^{2} x_{2}\left(1-2 \pi^{2} t_{1}^{2}\right)} .
$$

Hence,

$$
\phi_{x_{1}} \sim \frac{-K^{2} c}{16 \pi^{2} x_{1}^{2} x_{2}} \text { and } \phi_{x_{2}} \sim \frac{-K^{2} c}{16 \pi^{2} x_{1} x_{2}^{2}} .
$$

Thus, the integral has no stationary phase unless

$$
|u|,|v| \sim \frac{K^{2} c^{2}}{T^{3}} .
$$

More precisely, if $u, v$ do not satisfy the above conditions, then

$$
\phi_{x_{1}}-\frac{u}{c}, \phi_{x_{2}}-\frac{v}{c} \sim \frac{c K^{2}}{T^{3}} .
$$

Integrating by parts $A$ times. The oscillatory integral is bounded by

$$
\left(\frac{T^{3}}{c K^{2}}\right)^{A}\left(\frac{1}{T}\right)^{A} \ll \frac{1}{c^{A}} .
$$

Hence the contribution is negligible if $c \gg K^{\epsilon}$ by taking $A$ large enough and the contribution from $c \ll K^{\epsilon}$ is also negligible by direct estimation.

Argue as in P.888 of [24],

$$
\begin{aligned}
& \phi_{x_{1} x_{2}}, \phi_{x_{2} x_{1}} \sim \frac{K^{2} c}{16 \pi^{2} x_{1}^{2} x_{2}^{2}} \sim \frac{K^{2} c}{T^{4}}, \phi_{x_{1} x_{1}} \sim \frac{K^{2} c}{8 \pi^{2} x_{1}^{3} x_{2}} \sim \frac{K^{2} c}{T^{4}} \\
& \phi_{x_{2} x_{2}} \sim \frac{K^{2} c}{8 \pi^{2} x_{1} x_{2}^{3}} \sim \frac{K^{2} c}{T^{4}} \text { and }\left|\frac{\partial\left(\phi_{x_{1}}, \phi_{x_{2}}\right)}{\partial\left(x_{1}, x_{2}\right)}\right| \gg \frac{K^{4} c^{2}}{T^{8}}
\end{aligned}
$$

Integrating by parts sufficiently many times, (3.3.6)

$$
\begin{aligned}
= & G \int_{0}^{\infty} \sum_{c \ll \frac{T^{2} K^{\epsilon} / 2}{K G}} c^{-\frac{5}{2}} \sum_{|u|,|v| \sim \frac{K^{2} c^{2}}{T^{3}}} \sum_{a, b(c)} S\left(a^{2}, b^{2} ; c\right) \times \\
& e\left(\frac{2 a b+u a+v b}{c}\right) \iint_{R_{T}} h^{\left(p_{1}\right)}(z) \partial_{1}^{p_{2}} \tilde{V}\left(G z+K-1, x_{1}\right) \times \\
& \partial_{1}^{p_{3}} \tilde{V}\left(G z+K-1, x_{2}\right)\left|4 \pi^{2}+8 \pi^{4} t_{1}^{4}\right|^{-\frac{1}{2}} \times \\
& e\left(\phi_{u, v, c}\left(x_{1}, x_{2}, z\right)\right) e\left(\frac{-u x_{1}-v x_{2}}{c}\right) \frac{d x_{1} d x_{2}}{x_{1} x_{2}} d z+O\left(K^{-A}\right)
\end{aligned}
$$


for all $A>0$.

Applying Lemma 4.7 in [24], the integral is

$$
=O\left(\frac{1+\log K+\log c}{K^{2} c T^{-2}}\right) .
$$

Using (4.15) in [24],

$$
\begin{aligned}
& \sum_{a, b(c)} S\left(a^{2}, b^{2} ; c\right) e\left(\frac{2 a b+u a+v b}{c}\right) \\
= & \begin{cases}O\left(\left(v, c_{1}\right) c_{1}^{3 / 2} c_{2}^{5 / 2+\epsilon}\right) & \text { if }\left(v, c_{1}\right) \mid u \\
0 & \text { otherwise }\end{cases}
\end{aligned}
$$

by writing $c=c_{1} c_{2},\left(2, c_{1}\right)=1$ and $c_{2} \mid 2^{\infty}$.

Putting all these together, (3.3.6)

$$
\begin{aligned}
& \ll G K^{\epsilon} \sum_{c \ll \frac{T^{2} K^{\epsilon} / 2}{K G}} c^{-\frac{5}{2}} \sum_{\substack{|u|,|v| \sim \frac{K^{2} c^{2}}{\left(v, c_{1}\right) \mid u} \\
T^{3}}}\left(v, c_{1}\right) c_{1}^{\frac{3}{2}} c_{2}^{\frac{5}{2}+\epsilon} K^{-2} c^{-1} T^{2}+O\left(G K^{\epsilon}\right) \\
& \ll G K^{\epsilon} \sum_{c_{1} \ll \frac{T^{2} K}{K G}} \frac{c_{1}^{\frac{\epsilon}{2}}}{K^{-\frac{5}{2}-1+\frac{3}{2}} T^{2}}\left(\frac{K^{2} c_{1}^{2}}{T^{3}}\right)^{2}+O\left(G K^{\epsilon}\right) \\
& \ll \frac{G K^{2+\epsilon}}{T^{4}} \sum_{\substack{c_{1} \ll \frac{T^{2} K^{\frac{\epsilon}{2}}}{K G}\\
}} c_{1}^{2}+O\left(G K^{\epsilon}\right) \\
& \ll G K^{\epsilon} \frac{T^{2}}{K G^{3}}+O\left(G K^{\epsilon}\right)
\end{aligned}
$$

Summing over $T=2^{t},\left(\frac{2}{3}-\epsilon\right) \log _{2} K \leq t \leq(1+2 \epsilon) \log _{2} K-1$. The resulting sum is less than or equal to $G K^{\epsilon}$ whenever $G \geq K^{\frac{1}{3}}$ as is desired. 


\title{
CHAPTER 4
}

\section{THE FIRST MOMENT OF THE CENTRAL VALUES OF THE RANKIN-SELBERG $L$-FUNCTIONS}

\author{
It is not the critic who counts; not the man \\ who points out how the strong man stumbles, \\ or where the doer of deeds could have done \\ them better. The credit belongs to the man who \\ is actually in the arena, whose face is marred \\ by dust and sweat and blood; who strives \\ valiantly; who errs, who comes short again and \\ again, because there is no effort without error \\ and shortcoming; but who does actually strive \\ to do the deeds; who knows great enthusiasms, \\ the great devotions; who spends himself in a \\ worthy cause; who at the best knows in the end \\ the triumph of high achievement, and who at \\ the worst, if he fails, at least fails while daring \\ greatly, so that his place shall never be with \\ those cold and timid souls who neither know \\ victory nor defeat.
}

-Theodore Roosevelt

In this chapter we will prove Theorem 1.2.6. 
It was shown by Luo and Sarnak in [25] (see P.787-P.789 in their paper) that

$$
\sum_{\substack{K \leq k \leq 2 K \\ k \text { even }}} \sum_{\substack{f_{k} \\ H_{k}}} \frac{L\left(\frac{1}{2}, \phi \otimes \mathrm{sym}^{2} f\right)}{L\left(1, \operatorname{sym}^{2} f\right)(k-1)} \ll_{\epsilon} K^{1+\epsilon} .
$$

Our result improved on it in the sense that that family we average over is of size as small as $\sim K^{\frac{4}{3}}$ instead of $\sim K^{2}$.

In this paper we first follow the strategy of [24] with the help of a few lemmas in [25] to arrive at an expression of roughly the form

$$
\begin{aligned}
& \sum_{c} \frac{1}{c} \sum_{\substack{t_{1} \sim T_{1} \\
t_{2} \sim T_{2}}} S\left(t_{1}^{2}, t_{2}^{2} ; c\right) e\left(\frac{2 t_{1} t_{2}}{c}\right) \frac{\lambda_{\phi}\left(t_{1}\right)}{\sqrt{t_{1}} t_{2}} \\
\times & \int_{0}^{\infty} g(y) \int_{-\infty}^{\infty} e\left(\frac{2 t_{1} t_{2}}{c}\left(-2 \pi^{2} t^{2}+\frac{2}{3} \pi^{4} t^{4}\right)+t y\right) d t d y
\end{aligned}
$$

with test function $g$ and $t_{1} t_{2}^{2} \ll K^{2+\frac{\epsilon}{4}}$. Then as in [25], we separate the arithmetic and analytic parts. Then comes the main departure from [24]. In [24], they dealt with the analytic part using the Poisson summation formula and estimated the sum of the Kloosterman sums (the arithmetic part) using evaluations of the Gauss sum. However in our situation, we can't use the Poisson summation formula, for the simple reason that $\lambda_{\phi}\left(t_{1}\right)$ is not a smooth function in $t_{1}$ and also that $t_{1}$ and $t_{2}$ might not be in the same range. Instead of the Poisson summation formula, we appeal to the Voronoi formula for the Maass cusp forms. The classical Voronoi formula is of roughly the same strength as the Poisson summation formula as is demonstrated in Section 4.5 of [12]. Our result can be seen as a demonstration of such principle in the $G L(2)$ case. On the other hand the arithmetic part is more delicate in our situation and require lengthy if not slightly tedious treatment.

In section 4.1 we apply the approximate functional equation and the Petersson trace formula; in section 4.2 we shorten the length of summation over $c$; section 4.3, 4.5, 4.6, 4.7 and 4.9 are the core of the analytic components of the proof, involving the 
stationary phase lemma, Van der Corput's Lemma and Voronoi's formula; section 4.4 and 4.8 are the core of the arithmetic components of the proof, consisting of estimating various twisted exponential sum; in section 4.10 we put everything together and finish the proof.

\subsection{First manipulation}

We start with the approximate functional equation

Proposition 4.1.1. (P.787 of [25])

$$
L\left(\frac{1}{2}, \phi \otimes \operatorname{sym}^{2} f\right)=2 \sum_{m, n \geq 1} \frac{\lambda_{\phi}(m) a_{F}(m, n)}{\sqrt{m n^{2}}} G_{k}\left(\pi^{3} m n^{2}\right)
$$

where

$$
G_{k}(x)=\frac{1}{2 \pi i} \int_{k} U_{k}(s)\left(\frac{4 x}{(k-1)^{2}}\right)^{-s} \frac{d s}{s}
$$

with

$$
U_{k}(s)=\left(1+T_{k}(s)\right) \frac{\Gamma\left(\left(s+\frac{1}{2}\right)+1+i t_{\phi}\right) \Gamma\left(\left(s+\frac{1}{2}\right)+1-i t_{\phi}\right)}{\Gamma\left(\frac{\frac{1}{2}+1+i t_{\phi}}{2}\right) \Gamma\left(\frac{\frac{1}{2}+1-i t_{\phi}}{2}\right)}
$$

and

$$
T_{k}(s)=\sum_{1 \leq r \leq 6} \frac{p_{r+1}(s)}{(k-1)^{r}}+O\left(\frac{(|s|+1)^{8}}{(k-1)^{7}}\right) ;
$$

$p_{r+1}(s)$ being polynomial of degree at most $r+1$.

Using the Hecke relation and the Petersson formula as in P.787-788 of [25], we 
have

$$
\begin{aligned}
& \sum_{k \text { even }} h\left(\frac{k-1-K}{G}\right) \sum_{f \in H_{k}} \frac{L\left(\frac{1}{2}, \phi \otimes \mathrm{sym}^{2} f\right)}{L\left(1, \mathrm{sym}^{2} f\right)(k-1)} \\
= & 2 \sum_{k \text { even }} h\left(\frac{k-K-1}{G}\right) \sum_{d=1}^{\infty} \frac{\mu(d)}{d^{\frac{3}{2}}} \sum_{s_{1}, s_{2}, t_{1}, t_{2} \geq 1} \frac{\lambda_{\phi}\left(d s_{1}^{2} t_{1}\right)}{\sqrt{s_{1}^{2} t_{1} s_{2}^{4} t_{2}^{2}}} \\
\times & G_{k}\left(\pi^{3} d^{3} s_{1}^{2} s_{2}^{4} t_{1} t_{2}^{2}\right)\left[\delta_{t_{1} t_{2}}+2 \pi i^{-k} \sum_{c \geq 1} \frac{S\left(t_{1}^{2}, t_{2}^{2} ; c\right)}{c} J_{k-1}\left(\frac{4 \pi t_{1} t_{2}}{c}\right)\right] \\
= & 2 \sum_{k \text { even }} h\left(\frac{k-K-1}{G}\right) \sum_{d=1}^{\infty} \frac{\mu(d)}{d^{\frac{3}{2}}} \sum_{s_{1}, s_{2}, t \geq 1} \frac{\lambda_{\phi}\left(d s_{1}^{2} t\right)}{s_{1} t^{\frac{3}{2}} s_{2}^{2}} G_{k}\left(\pi^{3} d^{3} s_{1}^{2} t^{3} s_{2}^{4}\right) \\
+ & \sum_{d \geq 1} \frac{\mu(d)}{\sum^{\frac{3}{2}}} \sum_{k \text { even } s_{1}, s_{2}, t_{1}, t_{2} \geq 1} \frac{\lambda_{\phi}\left(d s_{1}^{2} t_{1}\right) G_{k}\left(\pi^{3} d^{3} s_{1}^{2} s_{2}^{4} t_{1} t_{2}^{2}\right)}{s_{1} \sqrt{t_{1}} s_{2}^{2} t_{2}} \sum_{c \geq 1} \frac{S\left(t_{1}^{2}, t_{2}^{2} ; c\right)}{c} \\
\times & 4 \pi h\left(\frac{k-K-1}{G}\right) i^{-k} J_{k-1}\left(\frac{4 \pi t_{1} t_{2}}{c}\right) .
\end{aligned}
$$

Using the integral representation of $G_{k}(s)$, the diagonal term contributes (see P.788 of [25] for details)

$$
2 G L\left(1, \operatorname{sym}^{2} \phi\right) \int_{0}^{\infty} h(x) d x+O\left(G K^{-\frac{1}{2}+\epsilon}\right) .
$$

For the non-diagonal term, first notice that by the definition of $G_{k}(x)$ we can consider the $t_{1}, t_{2}$-terms such that $t_{1} t_{2}^{2} \ll K^{2+\epsilon}$ in which case the contribution from error term below is negligible) and now writing

$$
\begin{aligned}
& G_{k}(x) \\
= & H\left(\frac{4 x}{(k-1)^{2}}\right)+\sum_{1 \leq r \leq 6} \frac{1}{(k-1)^{r}} H_{r}\left(\frac{4 x}{(k-1)^{2}}\right)+O\left(\frac{1}{(k-1)^{7}}\right)
\end{aligned}
$$

where $H$ and $H_{r}, 1 \leq r \leq 6$, are smooth and decay exponentially as $x \rightarrow \infty$ (see P.789 of [25]), the non-diagonal term is

$$
\begin{aligned}
& \sum_{d \geq 1} \frac{\mu(d)}{d^{\frac{3}{2}}} \sum_{k \text { even } s_{1}, s_{2}, t_{1}, t_{2} \geq 1} \frac{\lambda_{\phi}\left(d s_{1}^{2} t_{1}\right)}{s_{1} \sqrt{t_{1}} s_{2}^{2} t_{2}} H\left(\frac{4 \pi^{3} d^{3} s_{1}^{2} t_{1} s_{2}^{4} t_{2}^{2}}{(k-1)^{2}}\right) \sum_{c \geq 1} \frac{S\left(t_{1}^{2}, t_{2}^{2} ; c\right)}{c} \\
\times & 4 \pi h\left(\frac{k-K-1}{G}\right) i^{-k} J_{k-1}\left(\frac{4 \pi t_{1} t_{2}}{c}\right)+O(1)
\end{aligned}
$$


Applying the bound $J_{k-1}(x) \ll\left(\frac{e x}{2 k}\right)^{k-1}$ (see (5.102) of [19]) we can reduce the range to $c \leq \frac{24 \pi t_{1} t_{2}}{K}$.

Let $\eta$ be a $C^{\infty}$ function such that

$$
\eta(x)= \begin{cases}1 & x \in(1,2) \\ 0 & x \notin\left(\frac{1}{2}, 3\right) ;\end{cases}
$$

$S(x)=\sum_{j \geq 0} \eta\left(\frac{x}{2^{j}}\right) ;$ and let for each $0 \leq j, g_{2^{j}}(x)=\frac{\eta\left(\frac{x}{2^{j}}\right)}{S(x)}$. Applying this smooth partition of unity, the non-diagonal term is

$$
\begin{aligned}
= & 4 \pi \sum_{T_{1}, T_{2} \mid 2^{\infty}} \sum_{d \geq 1} \frac{\mu(d)}{d^{\frac{3}{2}}} \sum_{s_{1}, s_{2}, t_{1}, t_{2} \geq 1} \frac{\lambda_{\phi}\left(d s_{1}^{2} t_{1}\right)}{s_{1} \sqrt{t_{1}} s_{2}^{2} t_{2}} g_{T_{1}}\left(t_{1}\right) g_{T_{2}}\left(t_{2}\right) \times \\
& \sum_{k \text { even }} H\left(\frac{4 \pi^{3} d^{3} s_{1}^{2} t_{1} s_{2}^{4} t_{2}^{2}}{(k-1)^{2}}\right) \sum_{c \leq \frac{24 \pi T_{1} T_{2}}{K}} \frac{S\left(t_{1}^{2}, t_{2}^{2} ; c\right)}{c} \\
\times & h\left(\frac{k-1-K}{G}\right) i^{-k} J_{k-1}\left(\frac{4 \pi t_{1} t_{2}}{c}\right)+O(1) .
\end{aligned}
$$

Since $H(x)$ decays exponentially as $x \rightarrow \infty$, we can consider instead

$$
\begin{aligned}
& 4 \pi \sum_{d \geq 1} \frac{\mu(d)}{d^{\frac{3}{2}}} \sum_{s_{1}, s_{2} \geq 1} \frac{1}{s_{1} s_{2}^{2}} \sum_{\substack{T_{1}, T_{2} \mid 2^{\infty} \\
d^{3} s_{1}^{2} T_{1} s_{2}^{4} T_{2}^{2} \ll K^{2+\frac{\epsilon}{4}}}} \sum_{t_{1}, t_{2} \geq 1} \frac{\lambda_{\phi}\left(d s_{1}^{2} t_{1}\right)}{\sqrt{t_{1}} t_{2}} g_{T_{1}}\left(t_{1}\right) g_{T_{2}}\left(t_{2}\right) \\
\times & \sum_{k \text { even }} H\left(\frac{4 \pi^{3} d^{3} s_{1}^{2} t_{1} s_{2}^{4} t_{2}^{2}}{(k-1)^{2}}\right) \sum_{c \leq \frac{24 \pi T_{1} T_{2}}{K}} \frac{S\left(t_{1}^{2}, t_{2}^{2} ; c\right)}{c} \\
\times & h\left(\frac{k-1-K}{G}\right) i^{-k} J_{k-1}\left(\frac{4 \pi t_{1} t_{2}}{c}\right)+O(1) .
\end{aligned}
$$

Using Lemma 4.2 in [24], (4.1.1) =

$$
\begin{aligned}
& 4 \pi \sum_{d \geq 1} \frac{\mu(d)}{d^{\frac{3}{2}}} \sum_{s_{1}, s_{2} \geq 1} \frac{1}{s_{1} s_{2}^{2}} \sum_{\substack{T_{1}, T_{2} \mid 2^{\infty} \\
d^{3} s_{1}^{2} T_{1} s_{2}^{4} T_{2}^{2} \ll K^{2+\frac{\epsilon}{4}}}} \sum_{t_{1}, t_{2} \geq 1} \frac{\lambda_{\phi}\left(d s_{1}^{2} t_{1}\right)}{\sqrt{t_{1}} t_{2}} g_{T_{1}}\left(t_{1}\right) g_{T_{2}}\left(t_{2}\right) \\
\times & \sum_{c \leq \frac{24 \pi T_{1} T_{2}}{K}} \frac{S\left(t_{1}^{2}, t_{2}^{2} ; c\right)}{c} \int_{-\infty}^{\infty} \hat{f}(t) \sin \left(\frac{4 \pi t_{1} t_{2}}{c} \cos 2 \pi t\right) d t+O(1)
\end{aligned}
$$


where

$$
f(t)=h\left(\frac{t-K}{G}\right) H\left(\frac{4 \pi^{3} d^{3} s_{1}^{2} t_{1} s_{2}^{4} t_{2}^{2}}{t^{2}}\right)
$$

\subsection{Further reduction to the sum over $c$}

We assert that we can restrict the $c$-sum to those $c \ll \frac{T_{1} T_{2} K^{\frac{\epsilon}{2}}}{K G}$. To this end, we consider the Taylor expansion of $\cos 2 \pi t$, take the first two terms and treat the rest trivially, precisely, let $x=\frac{2 \pi t_{1} t_{2}}{c}$ then for each $N \in \mathbb{N}$,

$$
\begin{aligned}
& \sin (x \cos 2 \pi t)=\sin \left(x\left(1-2 \pi^{2} t^{2}\right)\right) \sum_{\substack{0 \leq n, m \leq N-1 \\
+}} c_{n, m}\left(x t^{4}\right)^{2 n} t^{2 m} \\
& +\cos \left(x\left(1-2 \pi^{2} t^{2}\right)\right) \sum_{\substack{1 \leq n \leq N \\
0 \leq m \leq N-1}} d_{n, m}\left(x t^{4}\right)^{2 n-1} t^{2 m} \\
& +\quad O\left(\max \left\{\left(x t^{4}\right)^{2 N},\left(x t^{4}\right)^{4 N}\right\}\right)+O\left(\max \left\{t^{2 N}, t^{4 N}\right\}\right) .
\end{aligned}
$$

The largest error term gives

$$
\int_{-\infty}^{\infty}\left|\hat{f}(t) x t^{4}\right| d t \ll|x| \int_{-\infty}^{\infty}\left|\widehat{f^{(4)}}(t)\right| d t
$$

where $f(t)=h\left(\frac{t-K}{G}\right) H\left(\frac{4 \pi^{3} d^{3} s_{1}^{2} t_{1} s_{2}^{4} t_{2}^{2}}{t^{2}}\right)$.

Note that

$$
\begin{aligned}
& \frac{d^{j}}{d t^{j}} h\left(\frac{t-K}{G}\right) \ll_{j} G^{-j} \text { and } \\
& \frac{d^{j}}{d t^{j}} H\left(\frac{4 \pi^{3} d^{3} s_{1}^{2} t_{1} s_{2}^{4} t_{2}^{2}}{t^{2}}\right) \ll_{j}\left(\frac{4 \pi^{3} d^{3} s_{1}^{2} s_{2}^{4} t_{1} t_{2}^{2}}{t^{2}}\right)^{j} t^{-j} .
\end{aligned}
$$

Since $\frac{24 \pi T_{1} T_{2}}{K} \geq c \geq 1$ and $\frac{4 \pi^{3} d^{3} s_{1}^{2} s_{2}^{4} t_{1} t_{2}^{2}}{t^{2}} \ll K^{\epsilon}$, we have when $t \sim K$

$$
\frac{d^{j}}{d t^{j}} H\left(\frac{4 \pi^{3} d^{3} s_{1}^{2} t_{1} s_{2}^{4} t_{2}^{2}}{t^{2}}\right) \ll_{j, \epsilon} K^{-j} K^{\epsilon j}
$$


From which we conclude that

$$
\int_{-\infty}^{\infty}\left|\hat{f}(t) x t^{4}\right| d t \ll G^{-4}|x| \ll G^{-4} \frac{T_{1} T_{2}}{c}
$$

Under the assumption $c \gg \frac{T_{1} T_{2}}{K G} K^{\frac{\epsilon}{2}}$,

$$
\int_{-\infty}^{\infty}\left|\hat{f}(t) x t^{4}\right| d t \ll G^{-4} K^{-\frac{\epsilon}{2}} K G \leq K^{-\frac{\epsilon}{2}}
$$

whenever $K \geq G \geq K^{\frac{1}{3}}$.

Thus, under the assumption that $c \gg \frac{T_{1} T_{2}}{K G} K^{\frac{\epsilon}{2}}$, a typical integral one has to consider is

$$
\int_{-\infty}^{\infty} \hat{f}(t) \sin \left(x\left(1-2 \pi^{2} t^{2}\right)\right)\left(x t^{4}\right)^{l} d t, \int_{-\infty}^{\infty} \hat{f}(t) \cos \left(x\left(1-2 \pi^{2} t^{2}\right)\right)\left(x t^{4}\right)^{l} d t
$$

where $x$ and $f$ as before. Since the treatment of other terms are similar, we will only deal with

$$
\int_{-\infty}^{\infty} \hat{f}(t) \sin \left(x\left(1-2 \pi^{2} t^{2}\right)\right) d t
$$

here.

Our goal is then to show the above integral is negligible under our assumption. Applying the Fresenel integral (see Lemma 4.2 of [25])

$$
\begin{aligned}
& \int_{-\infty}^{\infty} \hat{f}(t) \sin \left(x\left(1-2 \pi^{2} t^{2}\right)\right) d t=\frac{1}{2 \sqrt{\pi}} \int_{0}^{\infty} f(\sqrt{2 y x}) \sin \left(y+x-\frac{\pi}{4}\right) \frac{d y}{\sqrt{y}} \\
= & \frac{1}{\sqrt{\pi}} \int_{0}^{\infty} h\left(\frac{\sqrt{2 y x}-K}{G}\right) H\left(\frac{4 \pi^{3} d^{3} s_{1}^{2} t_{1} s_{2}^{4} t_{2}^{2}}{(\sqrt{2 y x})^{2}}\right) \sin \left(y+x-\frac{\pi}{4}\right) \frac{d y}{\sqrt{y}} \\
= & \frac{1}{2 \sqrt{\pi}} \int_{0}^{\infty} h\left(\frac{\sqrt{2 y x}-K}{G}\right) H\left(\frac{\pi^{2} d^{3} s_{1}^{2} s_{2}^{4} t_{2} c}{y}\right) \frac{1}{\sqrt{y}} \sin \left(y+x-\frac{\pi}{4}\right) d y .
\end{aligned}
$$


Note that $K \leq x=\frac{2 \pi t_{1} t_{2}}{c} \ll K G K^{-\frac{\epsilon}{2}}, \sqrt{2 y x} \sim K$ and $d^{3} s_{1}^{2} s_{2}^{4} T_{1} T_{2}^{2} \ll K^{2+\frac{\epsilon}{4}}$. For each $j>0$,

$$
\begin{aligned}
& \frac{d^{j}}{d y^{j}} h\left(\frac{\sqrt{2 x y}-K}{G}\right) \\
& \ll \max \left\{\left(\frac{1}{G} \sqrt{\frac{x}{y}}\right)^{j}, \frac{1}{y}\left(\frac{1}{G} \sqrt{\frac{x}{y}}\right)^{j-1}, \cdots,\left(\frac{1}{y}\right)^{j-1}\left(\frac{1}{G} \sqrt{\frac{x}{y}}\right)\right\}, \\
& \frac{d^{j}}{d y^{j}} H\left(\frac{\pi^{3} d^{3} s_{1}^{2} s_{2}^{4} t_{2} c}{y}\right) \\
& \ll \max \left\{\left(\frac{d^{3} s_{1}^{2} s_{2}^{4} t_{2} c}{y^{2}}\right)^{j},\left(\frac{d^{3} s_{1}^{2} s_{2}^{4} t_{2} c}{y^{2}}\right)^{j-1} \frac{1}{y}, \cdots, \frac{d^{3} s_{1}^{2} s_{2}^{4} t_{2} c}{y^{2}} \frac{1}{y^{j-1}}\right\}
\end{aligned}
$$

and

$$
\frac{d^{j}}{d y^{j}} y^{-\frac{1}{2}} \ll y^{-\frac{1}{2}-j}
$$

Since $y x \sim K^{2}, y^{-1} \sim \frac{x}{K^{2}} \ll \frac{G}{K} K^{-\frac{\epsilon}{2}} ; \frac{1}{G} \sqrt{\frac{x}{y}} \sim \frac{1}{G} \frac{x}{K} \ll K^{-\frac{\epsilon}{2}} ;$ and $\frac{d^{3} s_{1}^{2} s_{2}^{4} T_{2} c}{y^{2}} \sim$ $\frac{d^{3} s_{1}^{2} s_{2}^{4} T_{2} c x^{2}}{K^{4}} \sim \frac{d^{3} s_{1}^{2} s_{2}^{4} T_{2} T_{1}^{2} T_{2}^{2}}{K^{4} c} \ll \frac{d^{3} s_{1}^{2} s_{2}^{4} T_{2}^{2} T_{1}}{K^{4}} K G K^{-\frac{\epsilon}{2}} \ll \frac{G}{K} K^{-\frac{\epsilon}{4}}$.

Thus, the $j$-th derivative of all the three functions are $\ll K^{-\frac{\epsilon j}{4}}$. From which we conclude that the integral

$$
\frac{1}{2 \sqrt{\pi}} \int_{0}^{\infty} h\left(\frac{\sqrt{2 y x}-K}{G}\right) H\left(\frac{\pi^{2} d^{3} s_{1}^{2} s_{2}^{4} t_{2} c}{y}\right) \frac{1}{\sqrt{y}} \sin \left(y+x-\frac{\pi}{4}\right) d y
$$

is negligible whenever $c \gg \frac{T_{1} T_{2}}{K G} K^{\frac{\epsilon}{2}}$. This shows that we can restrict the sum over $c$ to those less than $\frac{T_{1} T_{2}}{K G} K^{\frac{\epsilon}{2}}$. 


\subsection{Applying the Stationary Phase Lemma and the Hecke relation}

Following P.882 of [24]) we can consider instead (all the others being similar)

$$
\begin{aligned}
& 4 \pi \sum_{d \geq 1} \frac{\mu(d)}{d^{\frac{3}{2}}} \sum_{s_{1}, s_{2} \geq 1} \frac{1}{s_{1} s_{2}^{2}} \sum_{\substack{T_{1}, T_{2} \mid 2^{\infty} \\
d^{3} s_{1}^{2} s_{2}^{4} T_{1} T_{2}^{2} \ll K^{2+\frac{\epsilon}{4}}}} \sum_{c \ll \frac{T_{1} T_{2} K^{\frac{\epsilon}{2}}}{K G}} \frac{1}{c} \sum_{t_{1}, t_{2}} S\left(t_{1}^{2}, t_{2}^{2} ; c\right) \\
\times & e\left(\frac{2 t_{1} t_{2}}{c}\right) \frac{\lambda_{\phi}\left(d s_{1}^{2} t_{1}\right)}{\sqrt{t_{1}} t_{2}} g_{T_{1}}\left(t_{1}\right) g_{T_{2}}\left(t_{2}\right) \int_{0}^{\infty} h\left(\frac{y-K-1}{G}\right) \\
\times & H\left(\frac{4 \pi^{3} d^{3} s_{1}^{2} t_{1} s_{2}^{4} t_{2}^{2}}{y^{2}}\right) \int_{-\infty}^{\infty} e\left(\frac{2 t_{1} t_{2}}{c}\left(-2 \pi^{2} t^{2}+\frac{2}{3} \pi^{4} t^{4}\right)+t y\right) d t d y
\end{aligned}
$$

with the cost of an admissible error term.

Using the fact that $H(x)$ decays exponentially as $x \rightarrow \infty$, we can restricts the $d$-, $s_{1^{-}}, s_{2^{-}}, T_{1^{-}}, T_{2^{-}}$sums to

$$
\sum_{d \ll K^{\frac{2}{3}+\epsilon}} \sum_{s_{2} \ll K^{1+\frac{\epsilon}{2}}} \sum_{\substack{T_{2} \mid 2^{\infty} \\ T_{2} \ll K^{1+\epsilon}}} \sum_{\substack{T_{1} \mid 2^{\infty} \\ T_{1} \ll \frac{K^{2+\frac{\epsilon}{4}}}{T_{2}^{2}}}} \sum_{\substack{s_{1} \geq 1 \\ d^{3} s_{1}^{2} s_{2}^{4} T_{1} T_{2}^{2} \ll K^{2+\frac{\epsilon}{4}}}} .
$$

Let

$$
\psi(x)=g_{T_{1}}(x) \int_{0}^{\infty} h\left(\frac{y-K-1}{G}\right) H\left(\frac{4 \pi^{3} d^{3} s_{1}^{2} s_{2}^{4} t_{2}^{2} x}{y^{2}}\right) \int_{-\infty}^{\infty} e(\kappa(t)) d t d y
$$

where $\kappa(t)=\frac{2 x t_{2}}{c}\left(-2 \pi^{2} t^{2}+\frac{2}{3} \pi^{4} t^{4}\right)+t y$. Then as a consequence of the stationary phase lemma (the proof will be given in the Section 4.9), we have

\section{Lemma 4.3.1.}

$$
\begin{gathered}
\psi(x)=\frac{e\left(-\frac{1}{8}\right) g_{T_{1}}(x)}{\sqrt{2 x}} \sqrt{\frac{c}{t_{2}}} \int_{0}^{\infty} h\left(\frac{y-K-1}{G}\right) H\left(\frac{4 \pi^{3} d^{3} s_{1}^{2} s_{2}^{4} t_{2}^{2} x}{y^{2}}\right) \\
\times \frac{e(\kappa(\gamma))}{\sqrt{\left|-4 \pi^{2}+8 \pi^{4} \gamma^{4}\right|}} d y+O\left(G\left(\frac{c}{T_{1} t_{2}}\right)^{\frac{3}{2}}\right)
\end{gathered}
$$

where $\gamma=\frac{2 y c}{x t_{2}}\left(1+o\left(x^{-1}\right)\right)$. 
Indeed as we will see in section $10, \gamma$ is the root of $\kappa(t)$ that is closest to 0 . By the Implicit Function Theorem, we get

$$
\frac{\partial^{n} \gamma}{\partial x^{n}}=(-1)^{n} \frac{2 n ! y c}{x^{n+1} t_{2}}\left(1+o\left(x^{-1}\right)\right)
$$

Let $\theta$ be such that $\lambda_{\phi}(n) \ll n^{\theta}$ then progress toward Ramanujan conjecture for the Maass form indicates that we can take $\theta=\frac{1}{4}$. The error term $O\left(G\left(\frac{c}{T_{1} t_{2}}\right)^{\frac{3}{2}}\right)$ contributes

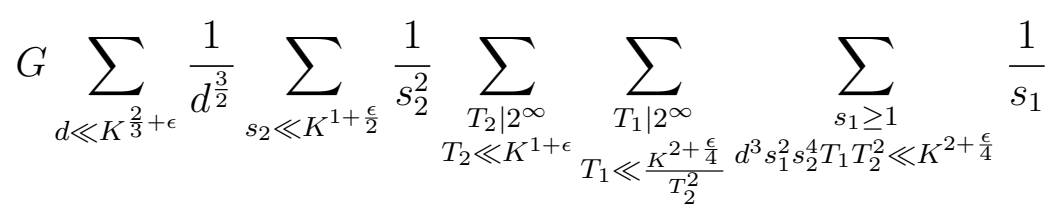

$$
\begin{aligned}
& \times \sum_{c \ll \frac{T_{1} T_{2} K^{\frac{\epsilon}{2}}}{K G}} c^{\frac{1}{2}} \sum_{\substack{t_{1} \sim T_{1} \\
t_{2} \sim T_{2}}} \frac{\left|S\left(t_{1}^{2}, t_{2}^{2} ; c\right)\right|\left|\lambda_{\phi}\left(d s_{1}^{2} t_{1}\right)\right|}{T_{1}^{2} T_{2}^{\frac{5}{2}}} \\
& \ll_{\epsilon} \quad G \sum_{d \ll K^{\frac{2}{3}+\epsilon}} d^{-\frac{3}{2}} \sum_{s_{2} \ll K^{1+\frac{\epsilon}{2}}} s_{2}^{-2} \sum_{\substack{T_{2} \mid 2^{\infty} \\
T_{2} \ll K^{1+\epsilon}}} \sum_{\substack{T_{1} \mid 2^{\infty} \\
T_{1} \ll \frac{K^{2+\frac{\epsilon}{4}}}{T_{2}^{2}}}} \sum_{\substack{s_{1} \geq 1 \\
d^{3} s_{1}^{2} s_{2}^{4} T_{1} T_{2}^{4} \ll K^{2+\frac{\epsilon}{4}}}} s_{1}^{-1} \\
& \times \sum_{c \ll \frac{T_{1} T_{2} K^{\frac{\epsilon}{2}}}{K G}} c^{1+\epsilon} \frac{\left(d s_{1}^{2} T_{1}\right)^{\theta}}{T_{1} T_{2}^{\frac{3}{2}}}
\end{aligned}
$$




$$
\begin{aligned}
& \ll G \sum_{d \ll K^{\frac{2}{3}+\epsilon}} d^{-\frac{3}{2}+\theta} \sum_{\substack{s_{2} \ll K^{1+\frac{\epsilon}{2}} \\
s_{2}^{-2}}} \sum_{\substack{T_{2} \mid 2^{\infty} \\
T_{2} \ll K^{1+\epsilon}}} \sum_{\substack{T_{1} \mid 2^{\infty} \\
T_{1} \ll \frac{K^{2+\frac{\epsilon}{4}}}{T_{2}^{2}}}} d^{3} s_{1}^{2} s_{2}^{4} T_{1} T_{2}^{4} \ll K^{2+\frac{\epsilon}{4}} \\
& s_{1}^{-1+2 \theta}\left(\frac{T_{1} T_{2} K^{\frac{\epsilon}{2}}}{K G}\right)^{2+\epsilon} T_{1}^{\theta-1} T_{2}^{-\frac{3}{2}} \\
& \ll G \sum_{d \ll K^{\frac{2}{3}+\epsilon}} d^{-\frac{3}{2}+\theta} \sum_{s_{2} \ll K^{1+\frac{\epsilon}{2}}} s_{2}^{-2} \sum_{\substack{T_{2} \mid 2^{\infty} \\
T_{2} \ll K^{1+\epsilon}}} \sum_{\substack{T_{1} \mid 2^{\infty} \\
T_{2} \ll \frac{K^{2+\frac{\epsilon}{4}}}{T_{2}^{2}}}}\left(\frac{K^{2+\frac{\epsilon}{4}}}{d^{3} s_{2}^{4} T_{1} T_{2}^{4}}\right)^{\theta} \\
& \times \frac{T_{1}^{1+\theta+\epsilon} T_{2}^{\frac{1}{2}+\epsilon}}{K^{2} G^{2}} \\
& \ll G \sum_{d \ll K^{\frac{2}{3}+\epsilon}} d^{-\frac{3}{2}-2 \theta} \sum_{s_{2} \ll K^{1+\frac{\epsilon}{2}}} s_{2}^{-2-4 \theta} \sum_{\substack{T_{2} \mid 2^{\infty} \\
T_{2} \ll K^{1+\epsilon}}} \sum_{\substack{T_{1} \mid 2^{\infty} \\
T_{1} \ll \frac{K^{2+\frac{\epsilon}{4}}}{T_{2}^{2}}}} \frac{K^{2 \theta-2+\epsilon}}{G^{2}} \\
& \times \quad T_{1}^{1+\epsilon} T_{2}^{\frac{1}{2}-4 \theta+\epsilon} \\
& \ll G \frac{K^{2 \theta-2+\epsilon}}{G^{2}} K^{2+\epsilon}=G \frac{K^{2 \theta+2 \epsilon}}{G^{2}} \ll G\left(\frac{K^{\frac{1}{4}}}{G}\right)^{2} K^{2 \epsilon} \ll G K^{2 \epsilon},
\end{aligned}
$$

note that the Weil bound on the Kloosterman sum was deployed.

Applying Lemma 4.3.1, we get

$$
\begin{aligned}
& \sum_{\substack{d \ll K^{\frac{2}{3}+\epsilon}\\
}} \frac{\mu(d)}{d} \sum_{\substack{s_{2} \ll K^{1+\frac{\epsilon}{2}} \\
s_{2}^{2}}} \sum_{\substack{T_{2} \mid 2^{\infty} \\
T_{2} \ll K^{1+\epsilon}}} \sum_{\substack{T_{1} \mid 2^{\infty} \\
T_{1} \ll \frac{K^{2+\frac{\epsilon}{4}}}{T_{2}^{2}}}} \sum_{\substack{s_{1} \geq 1 \\
K^{2+\frac{\epsilon}{4}} \gg d^{3} s_{1}^{2} s_{2}^{4} T_{1} T_{2}^{2}}} c^{-\frac{1}{2}} \sum_{\substack{c \ll \frac{g_{1} T_{2} K^{\frac{\epsilon}{2}}}{K G} \\
t_{2} \geq 1}} \frac{g_{T_{2}}\left(t_{2}\right)}{t_{2}^{\frac{3}{2}}} \sum_{t_{1} \geq 1} S\left(t_{1}^{2}, t_{2}^{2} ; c\right) e\left(\frac{2 t_{1} t_{2}}{c}\right) \\
& \times \frac{\lambda_{\phi}\left(d s_{1}^{2} t_{1}\right)}{\left(d s_{1}^{2}\right)^{\frac{3}{2}}} \Psi\left(d s_{1}^{2} t_{1}\right)
\end{aligned}
$$

where

$$
\begin{aligned}
& \Psi(x)=\frac{g_{T_{1}}\left(\frac{x}{d s_{1}^{2}}\right)}{x} \int_{0}^{\infty} h\left(\frac{y-K-1}{G}\right) H\left(\frac{4 \pi^{3} d^{2} s_{2}^{4} t_{2}^{2} x}{y^{2}}\right) \\
& \times \frac{e(\Phi(x, y))}{\sqrt{\left|-4 \pi^{2}+8 \pi^{4} \gamma^{2}\right|}} d y
\end{aligned}
$$


with

$$
\Phi(x, y)=\frac{2 x t_{2}}{c d s_{1}^{2}}\left(-2 \pi^{2} \gamma^{2}+\frac{2}{3} \pi^{4} \gamma^{4}\right)+\gamma y \text { and } \gamma=\frac{y c d s_{1}^{2}}{2 x t_{2}}\left(1+o\left(x^{-1}\right)\right) \text {, }
$$

i.e. $\Psi(x)=\psi\left(\frac{x}{d s_{1}^{2}}\right)$.

Breaking $t_{1}$ into congruence classes modulo $c,(4.3 .1)$

$$
\begin{aligned}
& =\sum_{d \ll K^{\frac{2}{3}+\epsilon}} \frac{\mu(d)}{d} \sum_{\substack{s_{2} \ll K^{1+\frac{\epsilon}{2}} \\
s_{2}^{2}}} \frac{1}{\substack{T_{2} \mid 2^{\infty} \\
T_{2} \ll K^{1+\epsilon}}} \sum_{\substack{T_{1} \mid 2^{\infty} \\
T_{1} \ll \frac{K^{2+\frac{\epsilon}{4}}}{T_{2}^{2}}}} \sum_{\substack{s_{1} \geq 1 \\
K^{2+\frac{\epsilon}{4}} \gg d^{3} s_{1}^{2} s_{2}^{4} T_{1} T_{2}^{2}}} \\
& \frac{1}{\left(d s_{1}^{2}\right)^{\frac{3}{2}}} \sum_{c \ll \frac{T_{1} T_{2} K^{\frac{\epsilon}{2}}}{K G}} c^{-\frac{3}{2}} \sum_{t_{2} \geq 1} \frac{g_{T_{2}}\left(t_{2}\right)}{t_{2}^{\frac{3}{2}}} \sum_{u(c)} \sum_{a(c)} S\left(a^{2}, t_{2}^{2} ; c\right) \\
& \times e\left(\frac{2 a t_{2}+u a}{c}\right) \sum_{n \geq 1} \lambda_{\phi}\left(d s_{1}^{2} n\right) e\left(-\frac{u n}{c}\right) \Psi\left(d s_{1}^{2} n\right) \text {. }
\end{aligned}
$$

Let $d s_{1}^{2}=q_{1}^{r_{1}} \cdots q_{m}^{r_{m}}$ be the unique prime factorization. Then using the Hecke relation,

$$
\lambda_{\phi}\left(r_{1} r_{2}\right)=\sum_{d \mid\left(r_{1}, r_{2}\right)} \mu(d) \lambda_{\phi}\left(\frac{r_{1}}{d}\right) \lambda_{\phi}\left(\frac{r_{2}}{d}\right)
$$

we have

$$
\begin{gathered}
\sum_{n \geq 1} \lambda_{\phi}\left(q_{1}^{r_{1}} \cdots q_{m}^{r_{m}} n\right) e\left(-\frac{u n}{c}\right) \Psi\left(q_{1}^{r_{1}} \cdots q_{m}^{r_{m}} n\right) \\
=\sum_{\left(q_{1}, n\right)=1} \lambda_{\phi}\left(q_{1}^{r_{1}} \cdots q_{m}^{r_{m}} n\right) e\left(-\frac{u n}{c}\right) \Psi\left(q_{1}^{r_{1}} \cdots q_{m}^{r_{m}} n\right) \\
+\sum_{\left(q_{1}, n\right)=q_{1}} \lambda_{\phi}\left(q_{1}^{r_{1}} \cdots q_{m}^{r_{m}} n\right) e\left(-\frac{u n}{c}\right) \Psi\left(q_{1}^{r_{1}} \cdots q_{m}^{r_{m}} n\right) \\
=\lambda_{\phi}\left(q_{1}^{r_{1}}\right) \sum_{\left(q_{1}, n\right)=1} \lambda_{\phi}\left(q_{2}^{r_{2}} \cdots q_{m}^{r_{m}} n\right) e\left(-\frac{u n}{c}\right) \Psi\left(q_{1}^{r_{1}} \cdots q_{m}^{r_{m}} n\right) \\
+\lambda_{\phi}\left(q_{1}^{r_{1}}\right) \sum_{\left(q_{1}, n\right)=q_{1}} \lambda_{\phi}\left(q_{2}^{r_{2}} \cdots q_{m}^{r_{m}} n\right) e\left(-\frac{u n}{c}\right) \Psi\left(q_{1}^{r_{1}} \cdots q_{m}^{r_{m}} n\right) \\
-\lambda_{\phi}\left(q_{1}^{r_{1}-1}\right) \sum_{\left(q_{1}, n\right)=q_{1}} \lambda_{\phi}\left(q_{2}^{r_{2}} \cdots q_{m}^{r_{m}} \frac{n}{q_{1}}\right) e\left(-\frac{u n}{c}\right) \Psi\left(q_{1}^{r_{1}} \cdots q_{m}^{r_{m}} n\right)
\end{gathered}
$$




$$
\begin{aligned}
& =\lambda_{\phi}\left(q_{1}^{r_{1}}\right) \sum_{n \geq 1} \lambda_{\phi}\left(q_{2}^{r_{2}} \cdots q_{m}^{r_{m}} n\right) e\left(-\frac{u n}{c}\right) \Psi\left(q_{1}^{r_{1}} \cdots q_{m}^{r_{m}} n\right) \\
& -\lambda_{\phi}\left(q_{1}^{r_{1}-1}\right) \sum_{n \geq 1} \lambda_{\phi}\left(q_{2}^{r_{2}} \cdots q_{m}^{r_{m}} n\right) e\left(-\frac{u q_{1} n}{c}\right) \Psi\left(q_{1}^{r_{1}} \cdots q_{m}^{r_{m}} q_{1} n\right) .
\end{aligned}
$$

By induction, we have

$$
\begin{aligned}
& \sum_{n \geq 1} \lambda_{\phi}\left(d s_{1}^{2} n\right) e\left(-\frac{u n}{c}\right) \Psi\left(d s_{1}^{2} n\right)=\lambda_{\phi}\left(d s_{1}^{2}\right) \sum_{n \geq 1} \lambda_{\phi}(n) e\left(-\frac{u n}{c}\right) \Psi\left(d s_{1}^{2} n\right) \\
& -\sum_{j=1}^{m} \lambda_{\phi}\left(d s_{1}^{2} / q_{j}\right) \sum_{n \geq 1} \lambda_{\phi}(n) e\left(-\frac{u q_{j} n}{c}\right) \Psi\left(d s_{1}^{2} q_{j} n\right) \\
& +\quad(-1)^{2} \sum_{1 \leq j_{1} \neq j_{2} \leq m} \lambda_{\phi}\left(\frac{d s_{1}^{2}}{q_{j_{1}} q_{j_{2}}}\right) \sum_{n \geq 1} \lambda_{\phi}(n) e\left(-\frac{u q_{j_{1}} q_{j_{2}} n}{c}\right) \Psi\left(d s_{1}^{2} q_{j_{1}} q_{j_{2}} n\right) \\
& +\quad \cdots+(-1)^{m} \lambda_{\phi}\left(\frac{d s_{1}^{2}}{\prod_{j=1}^{m} q_{j}}\right) \sum_{n \geq 1} \lambda_{\phi}(n) e\left(-\frac{u \prod_{j=1}^{m} q_{j} n}{c}\right) \Psi\left(d s_{1}^{2} \prod_{j=1}^{m} q_{j} n\right) .
\end{aligned}
$$

There are $2^{m}$ terms where $m=\omega\left(d s_{1}^{2}\right)$ is the number of distinct prime factor of $d s_{1}$ which is of size at most $\frac{\log d s_{1}^{2}}{\log \log d s_{1}^{2}}$.

\subsection{Evaluation of the Sums of the Kloosterman sum}

For each $c$, we can write $c=c_{1} c_{2}$ where $\left(c_{1}, c_{2}\right)=1$ and $c_{2} \mid 2^{\infty}$, i.e. when $c_{2}$ is a power of 2 . In this section we will evaluate

$$
\sum_{a(c)} S\left(a^{2}, t_{2}^{2} ; c\right) e\left(\frac{2 a t_{2}+u a}{c}\right)
$$

and determine the conditions for which the above sum is non-zero. In particular, we will show that the above sum is zero unless

$$
\left(\left(t_{2}+\overline{4} u\right) u, c_{1}\right)=\prod_{i=1}^{l} p_{i}^{k_{i}-1}
$$

and $k_{i}$ odd for all $1 \leq i \leq l$, here $c_{1}=p_{1}^{k_{1}} \cdots p_{l}^{k_{l}}$ is the prime factorization of $c_{1}$. This is done via evaluation of the quadratic Gauss sum with prime power modulus. 
By P.59, (4.12) of [7], let $\overline{c_{1}}, \overline{c_{2}}$ be such that $\overline{c_{2}} c_{2} \equiv 1\left(c_{1}\right)$ and $\overline{c_{1}} c_{1} \equiv 1\left(c_{2}\right)$. Then we have

$$
\begin{gathered}
\sum_{a(c)} S\left(a^{2}, t_{2}^{2} ; c\right) e\left(\frac{2 a t_{2}+u a}{c}\right) \\
=\sum_{a(c)} S\left(a^{2} \overline{c_{2}}, t_{2}^{2} \overline{c_{2}} ; c_{1}\right) S\left(a^{2} \overline{c_{1}}, t_{2}^{2} \overline{c_{1}} ; c_{2}\right) e\left(\frac{2 a t_{2}+u a}{c_{1} c_{2}}\right) \\
=\sum_{a_{1}\left(c_{1}\right)} S\left(\left(a_{1} c_{2}+a_{2} c_{1}\right)^{2} \overline{c_{2}}, t_{2}^{2} \overline{c_{2}} ; c_{1}\right) S\left(\left(a_{1} c_{2}+a_{2} c_{1}\right)^{2} \overline{c_{1}}, t_{2}^{2} \overline{c_{1}} ; c_{2}\right) \\
\times e\left(\frac{2\left(a_{1} c_{2}+a_{2} c_{1}\right) t_{2}+u\left(a_{1} c_{2}+a_{2} c_{1}\right)}{c_{1} c_{2}}\right) \\
=\sum_{a_{1}\left(c_{1}\right)} S\left(a_{1}^{2} c_{2}, t_{2}^{2} \overline{c_{2}} ; c_{1}\right) S\left(a_{2}^{2} c_{1}, t_{2}^{2} \overline{c_{1}} ; c_{2}\right) \\
\times a_{2}\left(c_{2}\right) \\
e\left(\frac{2 a_{1} t_{2}+u a_{1}}{c_{1}}\right) e\left(\frac{2 a_{2} t_{2}+u a_{2}}{c_{2}}\right) \\
=\sum_{a_{2}\left(c_{2}\right)}\left[\sum_{a_{1}\left(c_{1}\right)} S\left(a_{1}^{2} c_{2}, t_{2}^{2} \overline{c_{2}} ; c_{1}\right) e\left(\frac{2 a_{1} t_{2}+u a_{1}}{c_{1}}\right)\right] \\
\times \quad S\left(a_{2}^{2} c_{1}, t_{2}^{2} \overline{c_{1}} ; c_{2}\right) e\left(\frac{2 a_{2} t_{2}+u a_{2}}{c_{2}}\right) .
\end{gathered}
$$

We now evaluate the sum over $c_{1}$

$$
\begin{gathered}
\sum_{a_{1}\left(c_{1}\right)} S\left(a_{1}^{2} c_{2}, t_{2}^{2} \overline{c_{2}} ; c_{1}\right) e\left(\frac{2 a_{1} t_{2}+u a_{1}}{c_{1}}\right) \\
=\sum_{a_{1}\left(c_{1}\right)} \sum_{\substack{\left(d, c_{1}\right)=1 \\
\left(j, c_{1}\right.}} e\left(\frac{a_{1}^{2} c_{2}}{c_{1}} d\right) e\left(\frac{t_{2}^{2} \overline{c_{2}}}{c_{1}} \bar{d}\right) e\left(\frac{2 a_{1}\left(t_{2}+\overline{2} u\right)}{c_{1}}\right) \\
=\sum_{\substack{\left(d, c_{1}\right)=1 \\
a_{1}\left(c_{1}\right)}} \sum_{\substack{\left(d\left(c_{1}\right) \\
\left(d, c_{1}\right)=1\right.}} e\left(\frac{\left[a_{1} c_{2}+\left(t_{2}+\overline{2} u\right) \bar{d}\right]^{2} \overline{c_{2}} d}{\epsilon_{1}}\right) e\left(\frac{-\left(t_{2}+\overline{2} u\right)^{2} \overline{d c_{2}}+t_{2}^{2} \overline{c_{2} d}}{c_{1}}\right) \\
=\epsilon_{c_{1} \sqrt{c_{1}}} \sum_{\substack{d\left(c_{1}\right) \\
\left(d, c_{1}\right)=1}}\left(\frac{d}{c_{1}}\right)_{L} e\left(\frac{-\left(t_{2}+\overline{4} u\right) u \overline{c_{2}^{2}} d}{c_{1}}\right)
\end{gathered}
$$


since $\left(\frac{d}{c_{1}}\right)_{L}=\left(\frac{\bar{d}}{c_{1}}\right)_{L}$ where $(\dot{-})_{L}$ is the Jacobi-Legendre symbol and

$$
\epsilon_{c}= \begin{cases}1 & \text { if } c \equiv(4) \\ i & \text { if } c \equiv-1(4) .\end{cases}
$$

Hence,

$$
\begin{gathered}
\sum_{a_{1}\left(c_{1}\right)} S\left(a_{1}^{2} c_{2}, t_{2}^{2} \overline{c_{2}} ; c_{1}\right) e\left(\frac{2 a_{1} t_{2}+u a_{1}}{c_{1}}\right) \\
=\epsilon_{c_{1}} \sqrt{c_{1}} G\left(-\left(t_{2}+\overline{4} u\right) u \overline{c_{2}^{2}} ; c_{1}\right)
\end{gathered}
$$

where

$$
G\left(\beta, c_{1}\right)=\sum_{\substack{d\left(c_{1}\right) \\\left(d, c_{1}\right)=1}}\left(\frac{d}{c_{1}}\right)_{L} e\left(\frac{\beta d}{c_{1}}\right)
$$

is the quadratic Gauss sum.

Write $c_{1}=p_{1}^{k_{1}} \cdots p_{l}^{k_{l}}$ where $p_{1}, \cdots, p_{l}$ are distinct primes. Then by Lemma 3 in $[8]$,

$$
G\left(\beta, c_{1}\right)=\prod_{i} G\left(\beta \overline{\prod_{j \neq i} p_{j}^{k_{j}}} ; p_{i}^{k_{i}}\right) .
$$

Lemma 4.4.1. Let $p$ be an odd prime number, $(n, p)=1$ and $q=p^{k}$. Then

$$
G(n, q)=\left\{\begin{array}{l}
p^{k-\frac{1}{2}} \epsilon_{p}\left(\frac{n / p^{k-1}}{p}\right) \text { if } k \geq 1 \text { is odd and }\left(n, p^{k}\right)=p^{k-1} \\
0 \text { otherwise. }
\end{array}\right.
$$

Proof. By lemma 12.4 of [12], if $(n, p)=1$ then

$$
G(n, q)=\epsilon_{p} \sqrt{p}\left(\frac{n}{p}\right) \sum_{v^{2} \equiv 0(p)} e\left(\frac{2 v}{p}\right) .
$$

Thus if $k=1$, when $(n, p)=1$

$$
G(n, q)=\epsilon_{p} \sqrt{p}\left(\frac{n}{p}\right)_{L} .
$$

In the case when $(n, p)=p$ and $k=1$, a direct calculation shows that $G(n, q)=0$. 
For $k$ even,

$$
\begin{gathered}
G\left(n, p^{k}\right)=\sum_{d\left(p^{k}\right)}\left(\frac{d}{p}\right)_{L}^{k} e\left(\frac{n d}{p^{k}}\right) \\
=\sum_{d\left(p^{k}\right)} e\left(\frac{n d}{p^{k}}\right)=0 .
\end{gathered}
$$

For $k>1$ and odd, for each $0 \leq d<p^{k}$, write $d=u p+v$ where $0 \leq u<p^{k-1}$ and $0 \leq v<p$. Then

$$
\begin{aligned}
G\left(n, p^{k}\right)=\sum_{\substack{u\left(p^{k-1}\right) \\
v(p)}}\left(\frac{v}{p}\right)_{L} e\left(\frac{n(u p+v)}{p^{k}}\right) \\
=\sum_{v(p)}\left(\frac{v}{p}\right)_{L} e\left(\frac{n v}{p^{k}}\right) \sum_{u\left(p^{k-1}\right)} e\left(\frac{u n}{p^{k-1}}\right) .
\end{aligned}
$$

Hence if $\left(n, p^{k}\right) \neq p^{k-1}, G(n, q)=0$. Otherwise,

$$
\begin{aligned}
G(n, q) & =p^{k-1} \sum_{v(p)}\left(\frac{v}{p}\right)_{L} e\left(\frac{n^{\prime} v}{p}\right) \\
& =p^{k-\frac{1}{2}} \epsilon_{p}\left(\frac{n^{\prime}}{p}\right)_{L}
\end{aligned}
$$

where $n^{\prime}=n /\left(n, p^{k}\right)$. This concludes the proof of our lemma.

Thus, we conclude that for

$$
\sum_{a_{1}\left(c_{1}\right)} S\left(a_{1}^{2} c_{2}, t_{2}^{2} \overline{c_{2}} ; c_{1}\right) e\left(\frac{2 a_{1} t_{2}+u a_{1}}{c_{1}}\right)
$$

to be non-zero, we must have $\left(-\left(t_{2}+\overline{4} u\right) u, c_{1}\right)=\prod_{i=1}^{l} p_{i}^{k_{i}-1}$ (this includes the case $\left.k_{i}=1\right)$, in which case

$$
\begin{gathered}
\sum_{a_{1}\left(c_{1}\right)} S\left(a_{1}^{2} c_{2}, t_{2}^{2} \overline{c_{2}} ; c_{1}\right) e\left(\frac{2 a_{1} t_{2}+u a_{1}}{c_{1}}\right) \\
=\epsilon_{c_{1}} \frac{c_{1}^{\frac{3}{2}}}{\sqrt{\prod_{i=1}^{l} p_{i}}} \prod_{i=1}^{l} \epsilon_{p_{i}} \prod_{i=1}^{l}\left(\frac{-\left(t_{2}+\overline{4} u\right) u \overline{\prod_{j \neq i} p_{j}^{k_{j}} / p_{i}^{k_{i}-1}}}{p_{i}}\right)_{L} .
\end{gathered}
$$




\subsection{Applying Voronoi formula}

We now apply the following Voronoi formula

Lemma 4.5.1. (Theorem 2 in [26]) Let $f \in C_{0}^{\infty}(\mathbb{R})$ be a smooth function with compact support in $(0, \infty)$, and for any integer $C \geq 1$ and $(a, C)=1$, we have

$$
\begin{aligned}
& \sum_{n \geq 1} \lambda_{\phi}(n) e\left(\frac{a n}{C}\right) f(n)=\frac{\pi i}{C \sinh t_{\phi}} \\
\times & \sum_{n \geq 1} \lambda_{\phi}(n) e\left(-\frac{\bar{a} n}{C}\right) \int_{0}^{\infty} f(x)\left(J_{2 i t_{\phi}}\left(\frac{4 \pi \sqrt{n x}}{C}\right)-J_{-2 i t_{\phi}}\left(\frac{4 \pi \sqrt{n x}}{C}\right)\right) d x \\
+ & \frac{4 \cosh t_{\phi}}{C} \sum_{n \geq 1} \lambda_{\phi}(-n) e\left(\frac{\bar{a} n}{C}\right) \int_{0}^{\infty} f(x) K_{2 i t_{\phi}}\left(\frac{4 \pi \sqrt{n x}}{C}\right) d x
\end{aligned}
$$

where

$$
\Delta \phi=\left(\frac{1}{4}+t_{\phi}^{2}\right) \phi
$$

and

$$
a \bar{a} \equiv 1(C)
$$

From Section 4 , for each $d s_{1}^{2}=q_{1}^{r_{1}} \cdots q_{m}^{r_{m}}$, we know that we have to deal with the sum of the form

$$
\sum_{n \geq 1} \lambda_{\phi}(n) e\left(-\frac{u \beta^{\prime} n}{c_{1}^{\prime} c_{2}^{\prime}}\right) \Psi\left(d s_{1}^{2} \beta n\right)
$$

where $\beta$ is a product of distinct prime factors of $d s_{1}^{2}$,

$$
\beta^{\prime}=\frac{\beta}{(\beta, c)}, c_{1}^{\prime}=\frac{c_{1}}{\left(\beta, c_{1}\right)} \text { and } c_{2}^{\prime}=\frac{c_{2}}{\left(\beta, c_{2}\right)} \text {. }
$$

We can't assume that $\left(u, c_{2}\right)=1$. This presents a minor difficulty and to overcome it we have to break the sum $\sum_{\substack{u(c) \\\left(u, c_{1}\right)=1}}$ into

$$
\sum_{\begin{array}{c}
u(c) \\
\left(u, c_{1}\right)=1
\end{array}}=\sum_{j=0}^{\infty} \sum_{\begin{array}{c}
u(c) \\
\left(u, c_{1}\right)=1 \\
\left(u, c_{2}^{\prime}\right)=2^{j}
\end{array}}
$$


Fix $\left(u, c_{2}^{\prime}\right)=2^{j}$. We now apply the Voronoi formula to

$$
\sum_{n \geq 1} \lambda_{\phi}(n) e\left(-\frac{u^{\prime} \beta^{\prime} n}{c_{1}^{\prime \prime} c_{2, j}^{\prime \prime}}\right) \Psi\left(d s_{1}^{2} \beta n\right)
$$

where

$$
c_{2, j}^{\prime \prime}=\frac{c_{2}^{\prime}}{\left(u, c_{2}^{\prime}\right)}, u^{\prime}=\frac{u}{\left(u, c_{2}^{\prime}\right)\left(u, c_{1}^{\prime}\right)} \text { and } c_{1}^{\prime \prime}=\frac{c_{1}^{\prime}}{\left(u, c_{1}^{\prime}\right)}
$$

and obtain

$$
\begin{aligned}
& \sum_{n \geq 1} \lambda_{\phi}(n) e\left(-\frac{u^{\prime} \beta^{\prime} n}{c_{1}^{\prime \prime} c_{2, j}^{\prime \prime}}\right) \Psi\left(d s_{1}^{2} \beta n\right)=\frac{\pi i}{c_{1}^{\prime \prime} c_{2, j}^{\prime \prime} \sinh t_{\phi}} \\
\times & \sum_{j=1}^{2} \sum_{n \geq 1} \lambda_{\phi}(n) e\left(\frac{\overline{u^{\prime} \beta^{\prime}} n}{c_{1}^{\prime \prime} c_{2, j}^{\prime \prime}}\right) \int_{0}^{\infty} \Psi\left(d s_{1}^{2} \beta x\right) J_{(-1)^{j} 2 i t_{\phi}}\left(\frac{4 \pi \sqrt{n x}}{c_{1}^{\prime \prime} c_{2, j}^{\prime \prime}}\right) d x \\
+ & \frac{4 \cosh t_{\phi}}{c_{1}^{\prime \prime} c_{2, j}^{\prime \prime}} \sum_{n \geq 1} \lambda_{\phi}(n) e\left(\frac{\overline{u^{\prime} \beta^{\prime} n}}{c_{1}^{\prime \prime} c_{2, j}^{\prime \prime}}\right) \int_{0}^{\infty} \Psi\left(d s_{1}^{2} \beta x\right) K_{2 i t_{\phi}}\left(\frac{4 \pi \sqrt{n x}}{c_{1}^{\prime \prime} c_{2, j}^{\prime \prime}}\right) d x
\end{aligned}
$$

First of all note that $K_{2 i t}(z) \ll \min \left\{1+|\log z|, z^{-\frac{1}{2}} e^{-z}\right\}$ (see P.2416 of [22]) and since $n \in \mathbb{N}, d s_{1}^{2} s_{2}^{4} T_{1} T_{2}^{2} \ll K^{2+\frac{\epsilon}{4}}, \frac{d s_{1}^{2} \beta x}{d s_{1}^{2}} \sim T_{1}$ and $c \ll \frac{T_{1} T_{2} K^{\frac{\epsilon}{2}}}{K G}$,

$$
\frac{4 \pi \sqrt{n x}}{c_{1}^{\prime \prime} c_{2, j}^{\prime \prime}} \gg \frac{\sqrt{T_{1}}}{\sqrt{d s_{1}^{2}} c} \gg \frac{s_{2}^{2} T_{1} T_{2}}{c K^{1+\epsilon}} \gg \frac{G}{K^{\frac{3 \epsilon}{2}}} .
$$

Thus, the term involving $K_{2 i t_{\phi}}\left(\frac{4 \pi \sqrt{n x}}{c_{1}^{\prime \prime} c_{2, j}^{\prime \prime}}\right)$ is negligible. Hence we only have to deal with terms involving the J-Bessel function.

\subsection{Reduction of the range of sum over $n$}

The main goal of this section is to reduce the range of the sum over $n$. This is done using the asymptotic expansion of the $J$-Bessel function and then integrating by parts to show the contribution from the large $n$ is negligible. The treatment for the $J_{2 i t_{\phi}}$ and $J_{-2 i t_{\phi}}$ are quite similar, we would thus only deal with the term involving $J_{2 i t_{\phi}}$ 
here. We will use the following asymptotic expansion for $J_{2 i t}(z)$ (see [4], 7.13.1, (3), P.87), for any complex $\nu>0,-\pi<\arg z<\pi$ and $B>1$,

$$
\begin{gathered}
J_{\nu}(z)=\left(\frac{\pi z}{2}\right)^{-\frac{1}{2}}\left\{\cos \left(z-\frac{\nu \pi}{2}-\frac{\pi}{4}\right)\left[\sum_{m=0}^{B-1} t_{m, \nu} z^{-2 m}+\left(|z|^{-2 B}\right)\right]\right. \\
\left.-\sin \left(z-\frac{\nu \pi}{2}-\frac{\pi}{4}\right)\left[\sum_{m=0}^{B-1} t_{m, \nu}^{\prime} z^{-2 m-1}+O\left(|z|^{-2 B-1}\right)\right]\right\}
\end{gathered}
$$

for some constants $t_{m, \nu}, t_{m, \nu}^{\prime}$.

Since $\Psi(x)$ is supported in $\left[\frac{1}{2} d s_{1}^{2} T_{1}, 3 d s_{1}^{2} T_{1}\right], c \ll \frac{T_{1} T_{2} K^{\frac{\epsilon}{2}}}{K G}$ and $d^{3} s_{1}^{2} s_{2}^{4} T_{1} T_{2}^{2} \ll$ $K^{2+\frac{\epsilon}{4}}$

$$
\frac{4 \pi \sqrt{n x}}{c_{1}^{\prime} c_{2, j}^{\prime \prime}} \gg \frac{\sqrt{n T_{1}}}{c \sqrt{d s_{1}^{2}}} \gg \sqrt{n} G K^{-\frac{5 \epsilon}{8}},
$$

the asymptotic expansion for the $J$-Bessel function stated above can be applied to the integral

$$
\int_{0}^{\infty} \Psi\left(d s_{1}^{2} \beta x\right) J_{2 i t_{\phi}}\left(\frac{4 \pi \sqrt{n x}}{c_{1}^{\prime} c_{2, j}^{\prime \prime}}\right) d x .
$$

The error terms in the asymptotic expansion contributes

$$
O\left(\int_{0}^{\infty}\left|\Psi\left(d s_{1}^{2} \beta x\right)\right|\left(\frac{4 \pi \sqrt{n x}}{c_{1}^{\prime} c_{2, j}^{\prime \prime}}\right)^{-A} d x\right)
$$

where $A=2 B$ or $2 B+1$.

We will now show that the error term is admissible. To this end, we need to bound $\Psi(x)$. It is useful later to have a good bound on the derivatives of $\Psi(x)$. Thus we prove that for each $p \geq 0$,

\section{Lemma 4.6.1.}

$$
\Psi^{(p)}(x) \ll_{p} \frac{G}{d s_{1}^{2} T_{1}}\left[\left(\frac{K^{\frac{\epsilon}{4}}}{T_{1}}\right)^{p}+\left(\frac{K^{2} c}{d s_{1}^{2} T_{1}^{2} T_{2}}\right)^{p}\right] \ll \frac{G}{d s_{1}^{2} T_{1}}\left(\frac{K^{2} c}{d s_{1}^{2} T_{1}^{2} T_{2}}\right)^{p} .
$$

Proof. First recall that

$$
\Psi(x)=\frac{g_{T_{1}}\left(\frac{x}{d s_{1}^{2}}\right)}{x} \int_{0}^{\infty} h\left(\frac{y-K-1}{G}\right) H\left(\frac{4 \pi^{3} d^{2} s_{2}^{4} t_{2}^{2} x}{y^{2}}\right)
$$




$$
\frac{e(\Phi(x, y))}{\left|-4 \pi^{2}+8 \pi^{4} \gamma^{2}\right|} d y
$$

with

$$
\Phi(x, y)=\frac{2 x t_{2}}{c d s_{1}^{2}}\left(-2 \pi^{2} \gamma^{2}+\frac{2}{3} \pi^{4} \gamma^{4}\right)+\gamma y
$$

and

$$
\gamma=\frac{y c d s_{1}^{2}}{2 x t_{2}}\left(1+o\left(x^{-1}\right)\right)
$$

Since $g_{T_{1}}(z)$ is supported in $\left[\frac{1}{2} T_{1}, 3 T_{1}\right]$ and $h$ is supported in $\left[\frac{1}{2}, \frac{5}{2}\right]$, we conclude that $y \sim K, x \in\left[\frac{1}{2} d s_{1}^{2} T_{1}, 3 d s_{1}^{2} T_{1}\right]$. Also we know a priori that $c \ll \frac{T_{1} T_{2} K^{\frac{\epsilon}{2}}}{K G}$. With these in mind,

$$
\begin{aligned}
& \gamma \ll \frac{K c d s_{1}^{2}}{T_{1} T_{2} d s_{1}^{2}} \ll \frac{K^{\frac{\epsilon}{2}}}{G} \\
& \frac{\partial^{p} \gamma}{\partial x^{p}} \ll \frac{K c d s_{1}^{2}}{x^{p} T_{2}} \ll \frac{K c d s_{1}^{2}}{\left(d s_{1}^{2} T_{1}\right)^{p} T_{2}}, \text { for each } p \geq 0 .
\end{aligned}
$$

Hence, for each $p>0$,

$$
\begin{aligned}
& \left(\frac{\partial \Phi}{\partial x}\right)^{p} \ll\left(\frac{K^{2} c d s_{1}^{2}}{\left(T_{1} d s_{1}^{2}\right)^{2} T_{2}}\right)^{p}=\left(\frac{K^{2} c}{T_{1}^{2} T_{2} d s_{1}^{2}}\right)^{p} \ll\left(\frac{K^{1+\frac{\epsilon}{2}}}{d s_{1}^{2} T_{1} G}\right)^{p} \\
& \frac{\partial^{p} \Phi}{\partial x^{p}} \ll\left(\frac{d s_{1}^{2} T_{1} T_{2}}{c d s_{1}^{2}}\right)\left(\frac{K c d s_{1}^{2}}{T_{1} T_{2} d s_{1}^{2}}\right)^{2} \cdot \frac{1}{\left(d s_{1}^{2} T_{1}\right)^{p}}+\frac{K^{2} c d s_{1}^{2}}{\left(d s_{1}^{2} T_{1}\right)^{p} T_{2}} \\
\ll & \frac{K^{2} c d s_{1}^{2}}{\left(d s_{1}^{2} T_{1}\right)^{p} G} .
\end{aligned}
$$

Since $d^{3} s_{1}^{2} s_{2}^{4} T_{1} T_{2}^{2} \ll K^{2+\frac{\epsilon}{4}}$, we get

$$
\frac{d^{2} s_{2}^{4} t_{2}^{2}}{y^{2}} \ll \frac{d^{2} s_{2}^{4} T_{2}^{2}}{K^{2}} \ll \frac{K^{2+\frac{\epsilon}{4}}}{K^{2} T_{1}}=\frac{K^{\frac{\epsilon}{4}}}{T_{1}} .
$$

Putting all these together concludes our proof.

The error term contributes (by Lemma 4.6.1)

$$
\begin{aligned}
& O\left(G\left(\frac{c_{1}^{\prime \prime} c_{2, j}^{\prime \prime}}{\sqrt{n d s_{1}^{2} T_{1}}}\right)^{A}\right)=O\left(G\left(\frac{T_{1} T_{2}}{\sqrt{n T_{1}} \sqrt{d s_{1}^{2}}} \frac{c}{T_{1} T_{2}}\right)^{A}\right) \\
= & O\left(G\left(\frac{\sqrt{T_{1}} T_{2}}{\sqrt{n} \sqrt{d s_{1}^{2}}} \frac{K^{\frac{\epsilon}{2}}}{K G}\right)^{A}\right)=O\left(G\left(\frac{K^{\epsilon}}{\sqrt{n} G}\right)^{A}\right)
\end{aligned}
$$


which is negligible when $A=2 B(2 \mathrm{~B}+1$ respectively) is large enough (say $B=2015)$.

We will only consider

$$
\begin{aligned}
& \int_{0}^{\infty} \Psi\left(d s_{1}^{2} \beta x\right)\left(\frac{\sqrt{n x}}{c_{1}^{\prime \prime} c_{2, j}^{\prime \prime}}\right)^{-\frac{1}{2}} \cos \left(\frac{4 \pi \sqrt{n x}}{c_{1}^{\prime \prime} c_{2, j}^{\prime \prime}}-\frac{i t_{\phi} \pi}{2}-\frac{\pi}{4}\right) d x \\
= & \frac{\sqrt{c_{1}^{\prime \prime} c_{2, j}^{\prime \prime}}}{n^{\frac{1}{4}}} \int_{0}^{\infty} \Psi\left(d s_{1}^{2} \beta x\right) x^{-\frac{1}{4}} \cos \left(\frac{4 \pi \sqrt{n x}}{c_{1}^{\prime \prime} c_{2, j}^{\prime \prime}}-\frac{i t_{\phi} \pi}{2}-\frac{\pi}{4}\right) d x
\end{aligned}
$$

since the treatment for the other terms are quite similar.

We now show that we can restrict the sum over $n$ to those $n \ll \frac{K^{4+\frac{\epsilon}{100}} c^{2} c_{1}^{\prime \prime 2} c_{2, j}^{\prime \prime 2} d s_{1}^{2}}{T_{1}^{3} T_{2}}$. Indeed, integrating by parts $p$ times,

$$
\begin{aligned}
& \frac{\sqrt{c_{1}^{\prime \prime} c_{2, j}^{\prime \prime}}}{n^{\frac{1}{4}}} \int_{0}^{\infty} \Psi\left(d s_{1}^{2} \beta x\right) x^{-\frac{1}{4}} \cos \left(\frac{4 \pi \sqrt{n x}}{c_{1}^{\prime \prime} c_{2, j}^{\prime \prime}}-\frac{i t_{\phi}}{2}-\frac{\pi}{4}\right) d x \\
& \ll \frac{\sqrt{c_{1}^{\prime \prime} c_{2, j}^{\prime \prime}}}{n^{\frac{1}{4}}} \int_{x \sim d s_{1}^{2} T_{1}}\left(\frac{K^{2} c}{T_{1}^{2} T_{2}}\right)^{p} x^{\frac{p}{2}}\left(\frac{c_{1}^{\prime \prime} c_{2, j}^{\prime \prime}}{\sqrt{n}}\right)^{p} d x \\
& \ll \frac{\sqrt{c_{1}^{\prime \prime} c_{2, j}^{\prime \prime}} d s_{1}^{2} T_{1}}{n^{\frac{1}{4}}}\left(\frac{K^{2} c c_{1}^{\prime \prime} c_{2, j}^{\prime \prime} \sqrt{d s_{1}^{2} T_{1}}}{T_{1}^{2} T_{2} \sqrt{n}}\right)^{p} \\
& =\frac{\sqrt{c_{1}^{\prime \prime} c_{2, j}^{\prime \prime}} d s_{1}^{2} T_{1}}{n^{\frac{1}{4}}}\left(\frac{K^{4} c^{2} c_{1}^{\prime \prime 2} c_{2, j}^{\prime \prime 2} d s_{1}^{2}}{T_{1}^{3} T_{2} n}\right)^{\frac{p}{2}} .
\end{aligned}
$$

If $\frac{K^{4} c^{2} c_{1}^{\prime \prime 2} c_{2, j}^{\prime \prime 2} d s_{1}^{2}}{T_{1}^{3} T_{2} n} \ll K^{-\frac{\epsilon}{100}}$ then the integral is negligible by taking $p$ large. Hence we can assume $\frac{K^{4} c^{2} c_{1}^{\prime \prime 2} c_{2, j}^{\prime \prime 2} d s_{1}^{2}}{T_{1}^{3} T_{2} n} \gg K^{-\frac{\epsilon}{100}}$. In this case, we get

$$
n \ll \frac{K^{4+\frac{\epsilon}{100}} c^{2} c_{1}^{\prime \prime 2} c_{2, j}^{\prime \prime 2} d s_{1}^{2}}{T_{1}^{3} T_{2}} .
$$

Putting all these together,for each $d s_{1}^{2}$ and $j$ we have to deal with

$$
\sum_{\substack{u(c) \\\left(u, c_{2}^{\prime}\right)=2^{j}}} \sum_{a(c)} S\left(a^{2}, t_{2}^{2} ; c\right) e\left(\frac{2 a t_{2}+u a}{c}\right)
$$




$$
\begin{aligned}
& \times \sum_{n \ll \frac{K^{4+\frac{\epsilon}{100} c^{2} c_{1}^{\prime \prime 2} c_{2, j}^{\prime \prime 2} d s_{1}^{2}}}{T_{1}^{3} T_{2}}} \lambda_{\phi}(n) e\left(\frac{\overline{u^{\prime} \beta^{\prime}} n}{c_{1}^{\prime \prime} c_{2, j}^{\prime \prime}}\right) \frac{1}{n^{\frac{1}{4}} \sqrt{c_{1}^{\prime \prime} c_{2, j}^{\prime \prime}}} \\
& \times \int_{0}^{\infty} \Psi\left(d s_{1}^{2} \beta x\right) x^{-\frac{1}{4}} \cos \left(\frac{4 \pi \sqrt{n x}}{c_{1}^{\prime \prime} c_{2, j}^{\prime \prime}}-\frac{i t_{\phi}}{2}-\frac{\pi}{4}\right) d x .
\end{aligned}
$$

By Section 4.5, (4.6.1)

$$
\begin{aligned}
& =\frac{\epsilon_{c_{1}} \prod_{i=1}^{l} \epsilon_{p_{i}} c_{1}^{\frac{3}{2}}}{\sqrt{c_{1}^{\prime \prime} c_{2, j}^{\prime \prime}} \sqrt{\prod_{i=1}^{l} p_{i}}} \prod_{i=1}^{l}\left(\frac{-\overline{\prod_{j \neq i} p_{j}^{k_{j}}}}{p_{i}}\right)_{L} \sum_{\substack{\epsilon \\
n \ll \frac{K^{4+} \frac{\epsilon}{100} c^{2} c_{1}^{\prime \prime 2} c_{2, j}^{\prime \prime 2} d s_{1}^{2}}{T_{1}^{3} T_{2}}}} \frac{\lambda_{\phi}(n)}{n^{\frac{1}{4}}} \\
& \times \sum_{a_{2}\left(c_{2}\right)} S\left(a_{2}^{2} c_{1}, t_{2}^{2} \overline{c_{1}} ; c_{2}\right) e\left(\frac{2 a_{2} t_{2}}{c_{2}}\right) \\
& \times \quad \sum_{(u, c)} \quad\left(\frac{\left(t_{2}+\overline{4} u\right) u /\left(p_{1}^{k_{1}-1} \cdots p_{l}^{k_{l}-1}\right)}{p_{1} \cdots p_{l}}\right)_{L} e\left(\frac{u a_{2}}{c_{2}}\right) \\
& \begin{aligned}
\left(\left(t_{2}+\overline{4} u\right) u, c_{1}\right) & =\prod_{i=1}^{l} p_{i}^{k_{i}-1} \\
\left(u, c_{2}\right) & =2^{j}
\end{aligned} \\
& \times e\left(\frac{\overline{u^{\prime} \beta^{\prime}} n}{c_{1}^{\prime \prime} c_{2, j}^{\prime \prime}}\right) \int_{0}^{\infty} \Psi\left(d s_{1}^{2} \beta x\right) x^{-\frac{1}{4}} \cos \left(\frac{4 \pi \sqrt{n x}}{c_{1}^{\prime \prime} c_{2, j}^{\prime \prime}}-\frac{i t_{\phi} \pi}{2}-\frac{\pi}{4}\right) d x .
\end{aligned}
$$

\subsection{Bounding the Oscillatory integral}

In this section we will apply the Van der Corput Lemma (see Lemma 4.7.1 below) to get a better bound on the oscillatory integral

$$
\int_{0}^{\infty} \Psi\left(d s_{1}^{2} \beta x\right) x^{-\frac{1}{4}} e\left( \pm \frac{2 \sqrt{n x}}{c_{1}^{\prime \prime} c_{2, j}^{\prime \prime}}\right) d x
$$

(and hence on $\left.\int_{0}^{\infty} \Psi\left(d s_{1}^{2} \beta x\right) x^{-\frac{1}{4}} \cos \left(\frac{4 \pi \sqrt{n x}}{c_{1}^{\prime \prime} c_{2, j}^{\prime \prime}}-\frac{i t_{\phi}}{2}-\frac{\pi}{4}\right) d x\right)$. In particular, we will show that

$$
\begin{gathered}
\int_{0}^{\infty} \Psi\left(d s_{1}^{2} \beta x\right) x^{-\frac{1}{4}} e\left( \pm \frac{2 \sqrt{n x}}{c_{1}^{\prime \prime} c_{2, j}^{\prime \prime}}\right) d x \\
\ll G\left(d s_{1}^{2}\right)^{-\frac{3}{4}} \frac{T_{1}^{\frac{1}{4}} \sqrt{T_{2}}}{\sqrt{c} K \sqrt{\beta}} .
\end{gathered}
$$


We will make heavy use of the following well-known Van der Corput lemma.

Lemma 4.7.1. Suppose that $\phi:[a, b] \rightarrow \mathbb{R}$ is a phase obeying the bound $\left|\phi^{(k)}(x)\right| \geq \lambda$ for all $x \in[a, b]$ for some $k \geq 2$ and $\lambda>0$, and $\psi:[a, b] \rightarrow \mathbb{C}$ is a function such that $\psi^{\prime} \in L^{1}[a, b]$ then

$$
\int_{a}^{b} e^{i \phi(x)} \psi(x) d x \ll \lambda^{-\frac{1}{k}}\left[|\psi(b)|+\int_{a}^{b}\left|\psi^{\prime}(x)\right| d x\right] .
$$

The same claim also holds for $k=1$ provided that $\phi^{\prime}(x)$ is monotone.

Recall that

$$
\begin{aligned}
& \int_{0}^{\infty} \Psi\left(d s_{1}^{2} \beta x\right) x^{-\frac{1}{4}} e\left( \pm \frac{2 \sqrt{n x}}{c_{1}^{\prime \prime} c_{2, j}^{\prime \prime}}\right) d x \\
= & \int_{0}^{\infty} h\left(\frac{y-K-1}{G}\right) \int_{x \sim d s_{1}^{2} T_{1}} H\left(\frac{4 \pi^{3} d^{2} s_{2}^{4} t_{2}^{2} d s_{1}^{2} \beta x}{y^{2}}\right) \frac{x^{-\frac{1}{4}}}{x \sqrt{\left|-4 \pi^{2}+8 \pi^{4} \gamma^{2}\right|}} \\
\times & e\left(\Phi\left(d s_{1}^{2} \beta x, y\right) \pm \frac{2 \sqrt{n x}}{c_{1}^{\prime \prime} c_{2, j}^{\prime \prime}}\right) d x d y
\end{aligned}
$$

where

$$
\Phi(x, y)=\frac{2 x t_{2}}{c d s_{1}^{2}}\left(-2 \pi^{2} \gamma^{2}+\frac{2}{3} \pi^{4} \gamma^{4}\right)+\gamma y
$$

and

$$
\gamma \sim \frac{y c d s_{1}^{2}}{2 x t_{2}}
$$

Then

$$
\begin{aligned}
& \frac{\partial \Phi}{\partial x}=\frac{2 t_{2}}{c d s_{1}^{2}}\left(-2 \pi^{2} \gamma^{2}+\frac{2}{3} \pi^{4} \gamma^{4}\right)+\frac{2 x t_{2}}{c d s_{1}^{2}}\left(-4 \pi^{2} \gamma+\frac{8}{3} \pi^{4} \gamma^{3}\right) \frac{\partial \gamma}{\partial x} \\
+ & \frac{\partial \gamma}{\partial x} y \\
\sim & \frac{-4 \pi^{2} t_{2}}{c d s_{1}^{2}}\left(\frac{K c d s_{1}^{2}}{2 x t_{2}}\right)^{2}+\frac{-8 \pi^{2} x t_{2}}{c d s_{1}^{2}}\left(\frac{K c d s_{1}^{2}}{2 x t_{2}}\right)\left(\frac{-K c d s_{1}^{2}}{2 x^{2} t_{2}}\right) \\
- & \frac{K^{2} c d s_{1}^{2}}{2 x^{2} t_{2}} \\
= & \left(\pi^{2}-\frac{1}{2}\right) \frac{K^{2} c d s_{1}^{2}}{x^{2} t_{2}}
\end{aligned}
$$


In the case of $\Phi\left(d s_{1}^{2} \beta x, y\right)+\frac{2 \sqrt{n x}}{c_{1}^{\prime \prime} c_{2, j}^{\prime \prime}}$, when $x \sim d s_{1}^{2} T_{1}$, by differentiating the function

$$
\frac{\partial}{\partial x} \Phi\left(d s_{1}^{2} \beta x, y\right)+\frac{\partial}{\partial x}\left(\frac{2 \sqrt{n x}}{c_{1}^{\prime \prime} c_{2, j}^{\prime \prime}}\right)
$$

with respect to $x$ we can see that it is monotonic and we get

$$
\frac{\partial}{\partial x} \Phi\left(d s_{1}^{2} \beta x, y\right)+\frac{\partial}{\partial x}\left(\frac{2 \sqrt{n x}}{c_{1}^{\prime \prime} c_{2, j}^{\prime \prime}}\right) \gg \frac{K^{2} c \beta}{T_{1}^{2} T_{2}}
$$

from the above calculation.

Thus, applying the Van der Corput Lemma,

$$
\begin{gathered}
\int_{0}^{\infty} \Psi\left(d s_{1}^{2} \beta x\right) x^{-\frac{1}{4}} e\left(\frac{2 \sqrt{n x}}{c_{1}^{\prime \prime} c_{2, j}^{\prime \prime}}\right) d x \\
\ll \frac{G}{d s_{1}^{2} T_{1}}\left(d s_{1}^{2} T_{1}\right)^{-\frac{1}{4}} \frac{T_{1}^{2} T_{2}}{K^{2} c \beta}=\frac{G T_{1}^{\frac{3}{4}} T_{2}}{c K^{2} \beta\left(d s_{1}^{2}\right)^{\frac{5}{4}}}
\end{gathered}
$$

which is superior to what is claimed in the beginning of the section.

In the case of $\Phi\left(d s_{1}^{2} \beta x, y\right)-\frac{2 \sqrt{n x}}{c_{1}^{\prime \prime} c_{2, j}^{\prime \prime}}$,

$$
\frac{\partial}{\partial x}\left(\Phi\left(d s_{1}^{2} \beta x, y\right)-\frac{2 \sqrt{n x}}{c_{1}^{\prime \prime} c_{2, j}^{\prime \prime}}\right)=\left(\pi^{2}-\frac{1}{2}\right) \frac{K^{2} c d s_{1}^{2} \beta}{x^{2} t_{2}}-\frac{\sqrt{n}}{c_{1}^{\prime \prime} c_{2, j}^{\prime \prime} \sqrt{x}}\left(1+o\left(x^{-1}\right)\right)
$$

which is bounded away from $\frac{K^{2} c \beta}{T_{1}^{2} T_{2}}$ unless

$$
\frac{K^{4} c^{2} c_{1}^{\prime \prime 2} c_{2, j}^{\prime \prime 2} d s_{1}^{2} \beta^{2}}{T_{1}^{3} T_{2}^{2}}\left(\pi^{2}-\frac{1}{2}\right)^{2} \frac{1}{27} \leq n \leq \frac{K^{4} c^{2} c_{1}^{\prime \prime 2} c_{2, j}^{\prime \prime 2} d s_{1}^{2} \beta^{2}}{T_{1}^{3} T_{2}^{2}}\left(\pi^{2}-\frac{1}{2}\right)^{2} 8
$$

when $\frac{d s_{1}^{2} T_{1}}{2} \leq x \leq 3 d s_{1}^{2} T_{1}$.

Thus, when $n$ is not in the above range we proceed as before using Van der Corput's Lemma which will yield the same upper bound for our oscillatory integral as before. When $n$ is in the above range, we again use the Van der Corput lemma. Note that

$$
\frac{\partial^{2}}{\partial x^{2}}\left(\Phi\left(d s_{1}^{2} \beta x, y\right)-\frac{2 \sqrt{n x}}{c_{1}^{\prime \prime} c_{2, j}^{\prime \prime}}\right)
$$




$$
=\left[-2\left(\pi^{2}-\frac{1}{2}\right) \frac{K^{2} c\left(d s_{1}^{2}\right)^{2} \beta}{x^{3} T_{2}}+\frac{\sqrt{n}}{2 x^{\frac{3}{2}} c_{1}^{\prime \prime} c_{2, j}^{\prime \prime}}\right]\left(1+o\left(x^{-1}\right)\right) .
$$

which is bounded below by

$$
\frac{K^{2} c \beta}{d s_{1}^{2} T_{1}^{3} T_{2}}
$$

in our range of $n$. Hence,

$$
\begin{aligned}
& \int_{0}^{\infty} \Psi(x) x^{-\frac{1}{4}} e\left(-\frac{2 \sqrt{n x}}{c_{1}^{\prime \prime} c_{2, j}^{\prime \prime}}\right) d x \ll \frac{G\left(d s_{1}^{2} T_{1}\right)^{-\frac{1}{4}}}{d s_{1}^{2} T_{1}} \sqrt{\frac{d s_{1}^{2} T_{1}^{3} T_{2}}{K^{2} c \beta}} \\
= & G\left(d s_{1}^{2}\right)^{-\frac{3}{4}} \frac{T_{1}^{\frac{1}{4}} \sqrt{T_{2}}}{\sqrt{c} K \sqrt{\beta}} .
\end{aligned}
$$

\subsection{Estimating the sum of twisted exponential sums}

The main purpose of this section is to estimate the sum of exponential sums twisted by the Jacobi-Legendre symbol. From Section 4.4, we know that for the sum in consideration to be non-zero we must have

$$
\left(\left(t_{2}+\overline{4} u\right) u, c_{1}\right)=p_{1}^{k_{1}-1} \cdots p_{l}^{k_{l}-1}
$$

with $k_{i}$ odd for all $i$. Let $q_{i}=c_{2} \prod_{j \neq i} p_{j}^{k_{j}}$.

Assume without loss of generality that $t_{2}<c_{1}$ (by taking $t_{2}\left(\bmod c_{1}\right)$ if necessary). For each $0 \leq u, t_{2}<c_{1}$, we write

$$
u=\sum_{i=1}^{l} x_{i} q_{i} \overline{q_{i}}+x c_{1}, 0 \leq x_{i}<p_{i}^{k_{i}}, 0 \leq x<c_{2}
$$

and

$$
t_{2}=\sum_{i=1}^{l} y_{i} q_{i} \overline{q_{i}}+y c_{1}, 0 \leq y_{i}<p_{i}^{k_{i}}, 0 \leq y<c_{2}
$$

such that $q_{i} \overline{q_{i}} \equiv 1\left(p_{i}^{k_{i}}\right)$ and $c_{1} \overline{c_{1}} \equiv 1\left(c_{2}\right)$. Then (4.8.1) is true if and only

$$
\left(\left(\sum_{i=1}^{l}\left(y_{i}+\overline{4} x_{i}\right) q_{i} \overline{q_{i}}+(x+y) c_{1}\right)\left(\sum_{i=1}^{l} x_{i} q_{i} \overline{q_{i}}+x c_{1}\right), p_{j}^{k_{j}}\right)=p_{j}^{k_{j}-1}
$$


for all $1 \leq j \leq l$. This is equivalent to

$$
\left(\left(y_{i}+\overline{4} x_{i}\right) x_{i}, p_{i}^{k_{i}}\right)=p_{i}^{k_{i}-1}
$$

for all $1 \leq i \leq l$, since $\left(q_{i}^{2} \overline{q_{i}^{2}}, p_{i}^{k_{i}}\right)=1$ for all $i$. We now state what (4.8.1) means to $x_{i}$.

Lemma 4.8.1. For each $1 \leq i \leq l$, (4.8.1) is equivalent to

(a) if $\left(y_{i}, p_{i}^{k_{i}}\right)=1$ then either

$$
x_{i}=p_{i}^{k_{i}-1} 4-4 y_{i}
$$

given that $k_{i}>1$ or

$$
x_{i}=p_{i}^{k_{i}-1} b
$$

for any $0<b<p_{i}$;

(b) if $\left(y_{i}, p_{i}^{k_{i}}\right)=p_{i}^{t}$ for some $0<t<\frac{k_{i}-1}{2}$ then

$$
x_{i}=p_{i}^{k_{i}-1-t} b
$$

for any $0<b<p_{i}$;

(c) if $\left(y_{i}, p_{i}^{k_{i}}\right)=p_{i}^{\frac{k_{i}-1}{2}}$, i.e. $y_{i}=p_{i}^{\frac{k_{i}-1}{2}}$ a for some $0<a<p_{i}$ then

$$
x_{i}=p_{i}^{\frac{k_{i}-1}{2}} b
$$

where $b \not \equiv-4 a\left(p_{i}\right)$;

$$
\begin{aligned}
& \text { (d) if }\left(y_{i}, p_{i}^{k_{i}}\right)=p_{i}^{t} \text { with } t>\frac{k_{i}-1}{2} \text { then } \\
& \qquad x_{i}=p_{i}^{\frac{k_{i}-1}{2}} b
\end{aligned}
$$

where $0<b<p_{i}$.

Proof. Since the proofs are similar in nature, we will only prove (b) and (c). Note also that $k_{i}$ is odd throughout the proofs below. 
(b) We first show that $p_{i} \mid x_{i}$. Assuming the contrary then

$$
\left(\left(y_{i}+\overline{4} x_{i}\right) x_{i}, p_{i}^{k_{i}}\right)=1
$$

which is a contradiction. Hence $x_{i}=p_{i}^{r} b$ for some $0<b<p_{i}$, then

$$
\left(y_{i}+\overline{4} x_{i}\right) x_{i}=\left(p_{i}^{t-\min \{t, r\}} a+\overline{4} p_{i}^{r-\min \{t, r\}} b\right) p_{i}^{r+\min \{r, t\}} b
$$

Since $2 t<k_{i}-2$ and $p_{i}^{2} \nmid p_{i}^{t-\min \{t, r\}} a+\overline{4} p_{i}^{r-\min \{t, r\}} b$, we must have $r \neq t$. Then either $t-\min \{r, t\} \neq 0$ or $r-\min \{r, t\} \neq 0$, in any case, $\left(p_{i}^{t-\min \{t, r\}} a+\overline{4} p_{i}^{r-\min \{t, r\}} b\right)=$ 1. Thus, $r+\min \{r, t\}=k_{i}-1$. We conclude in this case that $r=k_{i}-1-t$.

(c) Write $x_{i}=p_{i}^{r} b$ then

$$
\left(y_{i}+\overline{4} x_{i}\right) x_{i}=\left(p_{i}^{\frac{k_{i}-1}{2}-\min \left\{r, \frac{k_{i}-1}{2}\right\}} a+\overline{4} p_{i}^{r-\min \left\{\frac{k_{i}-1}{2}, r\right\}} b\right) p_{i}^{r+\min \left\{r, \frac{k_{i}-1}{2}\right\}} b .
$$

If $r<\frac{k_{i}-1}{2}$ then $r+\min \left\{r, \frac{k_{i}-1}{2}\right\}=2 r=k_{i}-1$ which is impossible.

If $r>\frac{k_{i}-1}{2}$ then $r+\min \left\{r, \frac{k_{i}-1}{2}\right\}=r+\frac{k_{i}-1}{2}>k_{i}-1$.

If $r=\frac{k_{i}-1}{2}$ and $b \not \equiv 4 p_{i}-4 a\left(p_{i}\right)$.

With Lemma 4.8.1, we can break $\quad \sum_{u(c)} \quad$ into a product of local sums.

$$
\begin{gathered}
\left(\left(t_{2}+\overline{4} u\right) u, c_{1}\right)=\prod_{i}^{l} p_{i}^{k_{i}-1} \\
\left(u, c_{2}\right)=2^{j}
\end{gathered}
$$

For example if $\left(y_{1}, p_{1}^{k_{1}}\right)=1$ and $\left(y_{i}, p_{i}^{k_{i}}\right)=p_{i}^{t}$ for some $t>\frac{k_{i}-1}{2}$ for all $i \geq 2$ then

$$
\sum_{\begin{array}{c}
u(c) \\
\left(\left(t_{2}+\overline{4} u\right) u, c_{1}\right)=\prod_{1 \leq i \leq l} p_{i}^{k_{i}-1} \\
\left(u, c_{2}\right)=2^{j}
\end{array}}=\sum_{\substack{x_{1}=p_{1}^{k_{1}-1} 4-4 y_{i} \\
x_{i}=p_{i} k_{i}-1 \\
0<b_{i}<p_{i} \\
\text { for all } i \geq 2 \\
\left(u, c_{2}\right)=2^{j}}}+\sum_{\begin{array}{c}
x_{i}=p_{i}-1 \\
0<b_{i}<p_{i} \\
\text { for all } i \\
\left(u, c_{2}\right)=2^{j} \\
\end{array}} .
$$

In each of these summands, $\operatorname{val}_{p_{i}}\left(c_{1}^{\prime \prime}\right)$ is the same. Thus the integral

$$
\int_{0}^{\infty} \Psi\left(d s_{1}^{2} \beta x\right) x^{-\frac{1}{4}} \cos \left(\frac{4 \pi \sqrt{n x}}{c_{1}^{\prime \prime} c_{2, j}^{\prime \prime}}-\frac{i t_{\phi} \pi}{2}-\frac{\pi}{4}\right) d x
$$


can be pulled out of each of the sum.

Note that

$$
\begin{aligned}
& \left(\frac{\left(t_{2}+\overline{4} u\right) u /\left(p_{1}^{k_{1}-1} \cdots p_{l}^{k_{l}-1}\right)}{p_{1} \cdots p_{l}}\right)_{L} e\left(\frac{u a_{2}}{c_{2}}\right) e\left(\overline{\frac{u^{\prime} \beta^{\prime}}{c_{1}^{\prime \prime} c_{2, j}^{\prime \prime}}}\right) \\
= & \prod_{i=1}^{l}\left(\frac{\left[\left(y_{i}+\overline{4} x_{i}\right) x_{i} / p_{i}^{k_{i}-1}\right] \delta_{i}}{p_{i}}\right)_{L} e\left(\frac{x c_{1} \overline{c_{1}} a_{2}}{c_{2}}\right) e\left(\frac{\bar{u}}{\frac{\overline{\left(u, c_{1}^{\prime}\right)} \beta^{\prime} n}{c_{1}^{\prime \prime} c_{2, j}^{\prime \prime}}}\right)
\end{aligned}
$$

for some $\left(\delta_{i}, p_{i}\right)=1$.

Write $c_{1}^{\prime}=\frac{c_{1}}{\left(\beta, c_{1}\right)}=p_{1}^{k_{1}^{\prime}} \cdots p_{l}^{k_{l}^{\prime}}$. Since $\left(u, c_{1}^{\prime}\right)=\prod_{i}\left(u, p_{i}^{k_{i}^{\prime}}\right)=\prod_{i}\left(x_{i}, p_{i}^{k_{i}^{\prime}}\right)$, we have

$$
\begin{aligned}
& \left(\frac{\left(t_{2}+\overline{4} u\right) u /\left(p_{1}^{k_{1}-1} \cdots p_{l}^{k_{l}-1}\right)}{p_{1} \cdots p_{l}}\right)_{L} e\left(\frac{u a_{2}}{c_{2}}\right) e\left(\overline{\frac{u^{\prime} \beta^{\prime}}{c_{1}^{\prime}} n}\right) \\
& =\prod_{i=1}^{l}\left(\frac{\left[\left(y_{i}+\overline{4} x_{i}\right) x_{i} / p_{i}^{k_{i}-1}\right] \delta_{i}}{p_{i}}\right)_{L} e\left(\frac{x a_{2}}{c_{2}}\right) \\
& \times e\left(\frac{\overline{\sum_{i} \frac{x_{i}}{\left(x_{i}, p_{i}^{\prime}\right)} \frac{q_{i} \bar{q}_{i}}{2^{j} \prod_{t \neq i}\left(x_{t}, p_{t}^{k_{t}^{\prime}}\right)}+\frac{x}{\left(x, 2^{j}\right)} c_{1} \overline{c_{1}}} \overline{\beta^{\prime}} n}{c_{1}^{\prime \prime} c_{2, j}^{\prime \prime}}\right) \\
& =\prod_{i=1}^{l}\left\{\left(\frac{\left[\left(y_{i}+\overline{4} x_{i}\right) x_{i} / p_{i}^{k_{i}-1}\right] \delta_{i}}{p_{i}}\right)_{L} e\left(\frac{\overline{\frac{x_{i}}{\left(x_{i}, p_{i}^{\prime}\right)}} \delta_{i}^{\prime} \overline{\beta^{\prime}} n}{p_{i}^{k_{i}^{\prime}}}\right)\right\} \\
& \times e\left(\frac{x a_{2}}{c_{2}}\right) e\left(\frac{\frac{x}{\frac{\left.x, 2^{j}\right)}{(x} \delta^{\prime} n}}{c_{2, j}^{\prime \prime}}\right)
\end{aligned}
$$

for some $\left(\delta_{i}^{\prime}, p_{i}^{k_{i}^{\prime}}\right)=1$ and $\left(\delta^{\prime}, c_{2, j}^{\prime \prime}\right)=1$.

We are going to estimate the local sum (the sum is over all $x_{i}$ satisfying the conditions on P.82)

$$
S_{i, n}:=\sum\left(\frac{\left[\left(y_{i}+\overline{4} x_{i}\right) x_{i} / p_{i}^{k_{i}-1}\right] \delta_{i}}{p_{i}}\right)_{L} e\left(\frac{\overline{\frac{x_{i}}{\left(x_{i}, p_{i}^{\prime}\right)}} \delta_{i}^{\prime} \overline{\beta^{\prime}} n}{p_{i}^{k_{i}^{\prime}}}\right)
$$

according to the different cases in Lemma 4.8.1.

Case 1: $\left(y_{i}, p_{i}^{k_{i}}\right)=1, k_{i}>1$ and $x_{i}=p_{i}^{k_{i}-1} 4-4 y_{i}$. 
In this case, the sum is over a single element. Hence,

$$
\left|S_{i, n}\right| \leq 1
$$

Case 2: $\left(y_{i}, p_{i}^{k_{i}}\right)=1$ and $x_{i}=p_{i}^{k_{i}-1} b, 0<b<p_{i}$ (see Lemma 4.4.1).

$$
\begin{aligned}
& S_{i, n}=\sum_{b=1}^{p_{i}-1}\left(\frac{\left.\delta_{i}\left(y_{i}+\overline{4} p_{i}^{k_{i}-1} b\right) b\right)}{p_{i}}\right)_{L} e\left(\frac{\bar{b} \delta_{i}^{\prime} \overline{\beta^{\prime}} n}{p_{i}^{k_{i}^{\prime}}}\right) \\
& \left\{\begin{array}{l}
=\sqrt{p_{i}} \text { if }\left(n, p_{i}^{k_{i}^{\prime}}\right)=p_{i}^{k_{i}^{\prime}-1} \\
=0 \text { if } k_{i}^{\prime}=1,\left(n, p_{i}\right)=p_{i} \\
\leq p_{i} \text { if } k_{i}^{\prime}>1 .
\end{array}\right.
\end{aligned}
$$

Case 3: $y_{i}=p_{i}^{t} a_{i}$ for some $0<t<\frac{k_{i}-1}{2}$.

$$
S_{i, n}=\sum_{b=1}^{p_{i}-1}\left(\frac{b\left(a_{i}+\overline{4} p_{i}^{k_{i}-1-2 t} b\right) \delta_{i}}{p_{i}}\right)_{L} e\left(\frac{\bar{b} \delta_{i}^{\prime} \overline{\beta^{\prime}} n}{p_{i}^{k_{i}^{\prime}}}\right) .
$$

Then,

$$
\left|S_{i, n}\right| \leq\left\{\begin{array}{l}
\sqrt{p_{i}} \text { if } k_{i}^{\prime}=1 \\
p_{i} \text { if } k_{i}^{\prime}>1
\end{array}\right.
$$

Case 4: $y_{i}=p_{i}^{\frac{k_{i}-1}{2}} a_{i}$ for some $0<a_{i}<p_{i}$.

$$
S_{i, n}=\left(\frac{\delta_{i}^{\prime}}{p_{i}}\right)_{L} \sum_{\substack{0<b<p_{i} \\ b \neq \equiv-4 a_{i}\left(p_{i}\right)}}\left(\frac{\left(a_{i} \bar{b}+\overline{4}\right) \delta_{i}}{p_{i}}\right)_{L} e\left(\frac{\bar{b} \delta_{i}^{\prime} \overline{\beta^{\prime}} n}{p_{i}^{\prime}}\right) .
$$

Then,

$$
\left|S_{i, n}\right| \leq\left\{\begin{array}{l}
\sqrt{p_{i}} \text { if } k_{i}^{\prime}=1,\left(n, p_{i}\right)=1 \\
1 \text { if } k_{i}^{\prime}=1,\left(n, p_{i}\right)=p_{i} \\
p_{i} \text { if } k_{i}^{\prime}>1 .
\end{array}\right.
$$

Case 5: $y_{=} p_{i}^{t} a_{i}$ for some $t>\frac{k_{i}-1}{2}$ and $0<a_{i}<p_{i}$.

$$
S_{i, n}=\left(\frac{\delta_{i}^{\prime}}{p_{i}}\right)_{L} \sum_{0<b<p_{i}} e\left(\frac{\bar{b} \delta_{i}^{\prime} \overline{\beta^{\prime}} n}{p_{i}^{k_{i}^{\prime}}}\right) .
$$


Hence,

$$
\left|S_{i, n}\right| \leq\left\{\begin{array}{l}
p_{i} \text { if } k_{i}^{\prime}=1,\left(n, p_{i}\right)=p_{i} \\
1 \text { if } k_{i}^{\prime}=1,\left(n, p_{i}\right)=1 \\
p_{i} \text { if } k_{i}^{\prime}>1 .
\end{array}\right.
$$

In case $2-5$, when $k_{i}^{\prime}>1$, we have $\left(x_{i}, p_{i}^{k_{i}^{\prime}}\right) \geq p_{i}$ and hence

$$
\left(c_{1}^{\prime \prime}, p_{i}^{k_{i}}\right) \leq \frac{\left(c_{1}, p_{i}^{k_{i}}\right)}{p_{i}}
$$

and

$$
c_{1}^{\prime \prime} \leq c_{1} \prod_{k_{i}^{\prime}>1} \frac{1}{p_{i}}
$$

\subsection{Proof of Lemma 4.4.1}

We need the following stationary phase lemma from [6] (Lemma 5.5.4 on P.114).

Lemma 4.9.1. Let $f(x)$ be a real function, four times continuously differentiable for $\beta_{1} \leq x \leq \beta_{2}$, and let $g(x)$ also be a real function, three times continuously differentiable for $\beta_{1} \leq x \leq \beta_{2}$. Suppose that there are positive parameters $M, N, S$ and $U$ with

$$
M \geq \beta_{2}-\beta_{1}, \quad N \geq \frac{M}{\sqrt{S}}
$$

and positive constants $C_{r}$ such that for $\beta_{1} \leq x \leq \beta_{2}$,

$$
\left|f^{(r)}(x)\right| \leq \frac{C_{r} S}{M^{r}},\left|g^{(s)}(x)\right| \leq \frac{C_{s} U}{N^{s}}
$$

for $2 \leq r \leq 4$ and $0 \leq s \leq 3$, and

$$
f^{\prime \prime}(x) \leq-\frac{S}{C_{2} M^{2}} \cdot\left(\operatorname{resp} . f^{\prime \prime}(x) \geq \frac{S}{C_{2} M^{2}}\right)
$$

Suppose also that $f^{\prime}(x)$ changes sign from positive (resp. negative) to negative 
(reps. positive) at a point $\gamma$ with $\beta_{1}<\gamma<\beta_{2}$. If $S$ is sufficiently large in terms of the constant $C_{r}$, then we have

$$
\begin{aligned}
& \int_{\beta_{1}}^{\beta_{2}} g(x) e(f(x)) d x=\frac{\left.g(\gamma) e\left(f(\gamma)-\frac{1}{8}\right)\right)}{\sqrt{\left|f^{\prime \prime}(\gamma)\right|}}\left(\operatorname{resp} . \frac{\left.g(\gamma) e\left(f(\gamma)+\frac{1}{8}\right)\right)}{\sqrt{\left|f^{\prime \prime}(\gamma)\right|}}\right) \\
+ & \sum_{j=1}^{2} \frac{(-1)^{j} g\left(\beta_{j}\right) e\left(f\left(\beta_{j}\right)\right)}{2 \pi i f^{\prime}\left(\beta_{j}\right)}\left(\operatorname{resp} \cdot \sum_{j=1}^{2} \frac{(-1)^{j-1} g\left(\beta_{j}\right) e\left(f\left(\beta_{j}\right)\right)}{2 \pi i f^{\prime}\left(\beta_{j}\right)}\right) \\
+ & O\left(\frac{M^{4} U}{S^{2}}\left(1+\frac{M}{N}\right)^{2}\left(\sum_{j=1}^{2} \frac{(-1)^{j-1}}{\left(\gamma-\beta_{j}\right)^{3}}\right)\right) \\
+ & O\left(\frac{M U}{S^{\frac{3}{2}}}\left(1+\frac{M}{N}\right)^{2}\right)
\end{aligned}
$$

Proof. To prove the respective statement in parenthesis we just have to take complex conjugate and notice that $-f$ satisfies the conditions in Lemma 5.5.4 in [6].

Lemma 4.9.2. For each $p \in \mathbb{N} \cup\{0\}$, let $\kappa(t)=z\left(-2 \pi^{2} t^{2}+\frac{2}{3} \pi^{4} t^{4}\right)+t y$, where $y \sim K, z \gg \frac{K G}{K^{\frac{\epsilon}{2}}}$. Then (i) the roots of $\kappa^{\prime}(t)$ are $\gamma_{1}=-\sqrt{\frac{3}{2}} \frac{1}{\pi}-t_{1}, \gamma:=\gamma_{2}=t_{2}$, $\gamma_{3}=\sqrt{\frac{3}{2}} \frac{1}{\pi}-t_{3}$ with $t_{j}:=t_{j}(y, z) \sim \frac{y}{z} \ll \frac{K^{\frac{\epsilon}{2}}}{G}$; (ii) let $\beta_{j}, j=1, \cdots, 4$ be such that $\beta_{1}<\gamma_{1}<\beta_{2}<\gamma_{2}<\beta_{3}<\gamma_{3}<\beta_{4}$; we have

$$
\begin{gathered}
\int_{-\infty}^{\infty} e(\kappa(t)) d t=\sum_{j=1}^{3} \frac{e\left(\kappa\left(\gamma_{j}\right)+(-1)^{j-1} \frac{1}{8}\right)}{\sqrt{\left|\kappa^{\prime \prime}\left(\gamma_{j}\right)\right|}} \\
+\sum_{j=1}^{4} \frac{(-1)^{j-1} e\left(\kappa\left(\beta_{j}\right)\right)}{\pi i \kappa^{\prime}\left(\beta_{j}\right)}+O\left(z^{-\frac{3}{2}}\right)
\end{gathered}
$$

Proof. (i) A simple consequence of intermediate value Theorem (see P.883 of [25]). (ii)

$$
\begin{aligned}
& \int_{-\infty}^{\infty} e(\kappa(t)) d t=\int_{\beta_{4}}^{\infty} e(\kappa(t)) d t+\sum_{j=1}^{3} \int_{\beta_{j}}^{\beta_{j+1}} e(\kappa(t)) d t \\
+ & \int_{-\infty}^{\beta_{1}} e(\kappa(t)) d t .
\end{aligned}
$$


We get the main term by applying Lemma 4.6.1 to each $\int_{\beta_{j}}^{\beta_{j+1}} e(\kappa(t)) d t$ with $g(t)=$ 1, $S=z, N=1, M=\beta_{j+1}-\beta_{j}, U=1, C_{r}=\left(\beta_{j+1}-\beta_{j}\right)^{r} 100 \pi^{4}$. We will now show that

$$
\int_{-\infty}^{\beta_{1}} e(\kappa(t)) d t=\frac{e\left(\kappa\left(\beta_{1}\right)\right)}{2 \pi i \kappa^{\prime}\left(\beta_{1}\right)}+O\left(z^{-2}\right)
$$

and

$$
\int_{\beta_{4}}^{\infty} e(\kappa(t)) d t=-\frac{e\left(\kappa\left(\beta_{4}\right)\right)}{2 \pi i \kappa^{\prime}\left(\beta_{4}\right)}+O\left(z^{-2}\right)
$$

Integrating by parts twice

$$
\begin{gathered}
\int_{-\infty}^{\beta_{1}} e(\kappa(t)) d t=\frac{e\left(\kappa\left(\beta_{1}\right)\right)}{2 \pi i \kappa^{\prime}\left(\beta_{1}\right)}+\frac{\kappa^{\prime \prime}\left(\beta_{1}\right) e\left(\kappa\left(\beta_{1}\right)\right)}{2 \pi i\left(\kappa^{\prime}\left(\beta_{1}\right)\right)^{3}} \\
-\frac{1}{(2 \pi i)^{2}} \int_{-\infty}^{\beta_{1}} \frac{d}{d t}\left(\frac{\kappa^{\prime \prime}(t)}{\left(\kappa^{\prime}(t)\right)^{3}}\right) \cdot e(\kappa(t)) d t \\
=\frac{e\left(\kappa\left(\beta_{1}\right)\right)}{2 \pi i \kappa^{\prime}\left(\beta_{1}\right)}+O\left(z^{-2}\right)
\end{gathered}
$$

Similarly,

$$
\int_{\beta_{4}}^{\infty} e(\kappa(t)) d t=-\frac{e\left(\kappa\left(\beta_{4}\right)\right)}{2 \pi i \kappa^{\prime}\left(\beta_{4}\right)}+O\left(z^{-2}\right)
$$

We now turn to the proof of Lemma 4.4.1. First of all, for each $j$ integrating by parts repeatedly shows that

$$
\begin{gathered}
\frac{g_{T_{1}}\left(\frac{x}{d s_{1}^{2}}\right) e\left(z\left(-2 \pi^{2} \beta_{j}^{2}+\frac{2}{3} \pi^{4} \beta_{j}^{4}\right)\right)}{\sqrt{x}} \int_{0}^{\infty} h\left(\frac{y-K-1}{G}\right) \\
\quad \times H\left(\frac{4 \pi^{3} d^{2} s_{2}^{2} t_{2}^{2} x}{y^{2}}\right) \frac{e\left(\beta_{j} y\right)}{z\left(-4 \pi^{2} \beta_{j}+\frac{8}{3} \pi^{4} \beta_{j}^{3}\right)+y} d y
\end{gathered}
$$


contributes $O\left(G^{-A}\right)$ for all $A>0$. Hence the contribution from

$$
\sum_{j=1}^{4} \frac{(-1)^{j-1} e\left(\kappa\left(\beta_{j}\right)\right)}{\pi i \kappa^{\prime}\left(\beta_{j}\right)}
$$

is negligible.

For $j \neq 2$, let

$$
\chi(y)=z\left(-2 \pi^{2} \gamma_{j}^{2}+\frac{2}{3} \pi^{4} \gamma_{j}^{4}\right)+\gamma_{j} y
$$

then differentiating with respect to $y$

$$
\chi^{\prime}(y)=\left[z\left(-4 \pi^{2} \gamma_{j}+\frac{8}{3} \pi^{4} \gamma_{j}^{3}\right)+y\right] \frac{\partial \gamma_{j}}{\partial y}+\gamma_{j} .
$$

Since $\kappa^{\prime}\left(\gamma_{j}\right)=0$, one gets $z\left(-4 \pi^{2} \gamma_{j}+\frac{8}{3} \pi^{4} \gamma_{j}^{3}\right)+y=0$ hence $\chi^{\prime}(y)=\gamma_{j} \asymp 1$. Integrating by parts repeatedly show that the integral involving $\gamma_{j}, j \neq 2$ contributes $O\left(K^{-A}\right)$ for all $A>0$. On the other hand $O\left(\left(\frac{T_{1} T_{2}}{c}\right)^{-\frac{3}{2}}\right)$ contributes

$$
\begin{aligned}
& O\left(\int_{0}^{\infty} h\left(\frac{y-K-1}{G}\right)\left|H\left(\frac{4 \pi^{3} d^{3} s_{1}^{2} s_{2}^{4} t_{2}^{2} T_{1}}{y^{2}}\right)\right|\left(\frac{c}{T_{1} T_{2}}\right)^{\frac{3}{2}}\right) \\
= & O\left(G\left(\frac{c}{T_{1} T_{2}}\right)^{\frac{3}{2}}\right) .
\end{aligned}
$$

This concludes the proof of Lemma 4.4.1 by taking $z=\frac{2 x t_{2}}{c}$.

\subsection{Concluding the proof of the main theorem}

For each $d s_{1}^{2}$ such that $d \ll K^{\frac{2}{3}+\frac{\epsilon}{2}}, s_{2} \ll K^{1+\frac{\epsilon}{2}}, T_{2}\left|2^{\infty}, T_{2} \ll K^{1+\epsilon}, T_{1}\right| 2^{\infty}, T_{1} \ll \frac{K^{2+\frac{\epsilon}{2}}}{T_{2}^{2}}$ and $d^{3} s_{1}^{2} s_{2}^{4} T_{1} T_{2}^{2} \ll K^{2+\frac{\epsilon}{4}}$. (4.6.1) is at most a sum of $\omega\left(c_{1}\right)$ (recall that $\omega(n)$ is the number of distinct prime factor of $n$ ), each of which is 


$$
\begin{aligned}
& \frac{c_{1}^{\frac{3}{2}} c_{2}^{\frac{5}{2}+\epsilon}}{\sqrt{c_{1}^{\prime \prime} c_{2, j}^{\prime \prime}} \sqrt{\prod_{i=1}^{l} p_{i}}} \frac{G\left(d s_{1}^{2}\right)^{-\frac{3}{4}} T_{1}^{\frac{1}{4}} \sqrt{T_{2}}}{\sqrt{c} K \sqrt{\beta}}\left[\sum_{n \ll \frac{K^{4+\frac{\epsilon}{100} c^{2} c_{1}^{\prime \prime 2} c_{2, j}^{\prime \prime 2} d s_{1}^{2}}}{T_{1}^{3} T_{2}}}^{\left(n,\left(t_{2}, c_{1}^{\prime}\right)\right)=1}\right.
\end{aligned}
$$

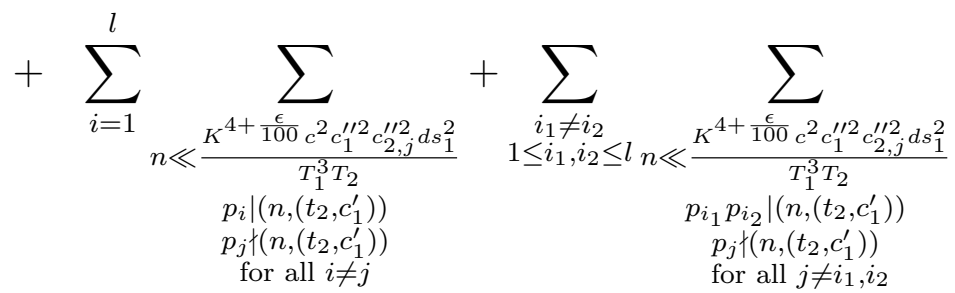

$$
\begin{aligned}
& \left.+\cdots+\sum_{\substack{n \ll \frac{K^{4+} \frac{\epsilon}{100} c^{2} c_{1}^{\prime \prime 2} c_{2, j}^{\prime \prime 2} d s_{1}^{2}}{T_{1}^{3} T_{2}} \\
p_{1} \cdots p_{l} \mid\left(n,\left(t_{2}, c_{1}^{\prime}\right)\right)}}\right] \frac{\left|\lambda_{\phi}(n)\right| \prod_{i}\left|S_{i, n}\right|}{n^{\frac{1}{4}}}
\end{aligned}
$$

Using the bound $\sum_{n \sim A}\left|\lambda_{\phi}(n)\right|^{2} \ll A$ (see P.246 (13.53) of [7]), and summing $\sum_{\substack{n \ll B \\ p_{i} \mid\left(n,\left(t_{2}, c_{1}^{\prime}\right)\right) \\ p_{j} \nmid\left(n,\left(t_{2}, c_{1}^{\prime}\right)\right) \\ \text { for all } i \neq j}}\left|\lambda_{\phi}(n)\right| n^{-\frac{1}{4}} \prod_{i}\left|S_{i, n}\right|$ dyadically,

$$
\begin{aligned}
& \sum_{\substack{\left.n \ll B \\
p_{i} \mid\left(n, t_{2}, c_{1}^{\prime}\right)\right) \\
p_{j} \nmid\left(n,\left(t_{2}, c_{1}^{\prime}\right)\right) \\
\text { for all } i \neq j}}\left|\lambda_{\phi}(n)\right| n^{-\frac{1}{4}} \prod\left|S_{i, n}\right|=\sum_{\substack{T \mid 2^{\infty} \\
T \ll B}} \sum_{\substack{n \sim T \\
p_{i} \mid\left(n,\left(t_{2}, c_{1}^{\prime}\right)\right) \\
p_{j} \nmid\left(n,\left(t_{2}, c_{1}^{\prime}\right)\right) \\
\text { for all } i \neq j}}\left|\lambda_{\phi}(n)\right| n^{-\frac{1}{4}} \prod\left|S_{i, n}\right| \\
& \leq \prod_{\substack{k_{j}^{\prime}>1 \\
j \neq i}} p_{j} \prod_{\substack{k_{j}^{\prime}=1 \\
j \neq i}} \sqrt{p_{j}} \cdot p_{i} \sum_{\substack{T \mid 2^{\infty} \\
T \ll B}} \sum_{\substack{n \sim T \\
p_{i} \mid\left(n,\left(t_{2}, c_{1}^{\prime}\right)\right) \\
p_{j} \nmid\left(n,\left(t_{2}, c_{1}^{\prime}\right)\right) \\
\text { for all } i \neq j}}\left|\lambda_{\phi}(n)\right| n^{-\frac{1}{4}} \\
& \ll \prod_{\substack{k_{j}^{\prime}>1 \\
j \neq i}} p_{j} \prod_{\substack{k_{j}^{\prime}=1 \\
j \neq i}} \sqrt{p_{j}} \cdot p_{i} \sum_{\substack{T \mid 2^{\infty} \\
T \ll B}} \frac{\left|\lambda_{\phi}\left(p_{i}\right)\right|}{T^{\frac{1}{4}}} \sqrt{\sum_{\substack{\left.\left.n, T \\
p_{i} \mid\left(n, t_{2}, c_{1}^{\prime}\right)\right) \\
p_{j} \nmid\left(n, t_{2}, c_{1}^{\prime}\right)\right) \\
\text { for all } i \neq j}}\left|\lambda_{\phi}(n)\right|^{2} \sum_{\substack{n \sim T \\
p_{i} \mid\left(n,\left(t_{2}, c_{1}^{\prime}\right)\right) \\
p_{j} \nmid\left(n,\left(t_{2}, c_{1}^{\prime}\right)\right) \\
\text { for all } i \neq j}} 1}
\end{aligned}
$$




$$
\begin{aligned}
& \ll \prod_{\substack{k_{j}^{\prime}>1 \\
j \neq i}} p_{j} \prod_{\substack{k_{j}^{\prime}=1 \\
j \neq i}} \sqrt{p_{j}} \cdot p_{i} \frac{B^{\frac{3}{4}}\left|\lambda_{\phi}\left(p_{i}\right)\right|}{p_{i}} \\
& \ll \prod_{\substack{k_{j}^{\prime}>1 \\
j \neq i}} p_{j} \prod_{\substack{k_{j}^{\prime}=1 \\
j \neq i}} \sqrt{p_{j}} \cdot \sqrt{p_{i}} B^{\frac{3}{4}}
\end{aligned}
$$

using bound toward Ramanujan's conjecture for Hecke -Maass forms (we use $\lambda_{\phi}(p) \ll$ $p^{\frac{1}{2}}$ here). It can be shown in a similar fashion that for any $1 \leq i_{1}, \cdots, i_{r} \leq l$,

$$
\sum_{\substack{n \ll B \\ p_{i} \cdots p_{i} \mid\left(n,\left(t_{2}, c_{1}^{\prime}\right)\right) \\ p_{j}\left(n,\left(t_{2}, c_{1}^{\prime}\right)\right) \\ \text { for all } j \neq i_{1}, \cdots, i_{r}}} \frac{\left|\lambda_{\phi}(n)\right|}{n^{\frac{1}{4}}} \prod\left|S_{i, n}\right| \ll \prod_{k_{i}^{\prime}>1} p_{i} \prod_{k_{i}^{\prime}=1} \sqrt{p_{i}} B^{\frac{3}{4}} .
$$

Hence using Weil's bound for the Kloosterman sum in dealing with the sum over $c_{2}$ and the fact that

$$
c_{1}^{\prime \prime} \leq c_{1} \prod_{k_{i}^{\prime}>1} \frac{1}{p_{i}}
$$

we have (4.10.1)

$$
\begin{aligned}
& \ll \frac{2^{\omega\left(c_{1}\right)} c_{1}^{\frac{3}{2}} c_{2}^{\frac{5}{2}+\epsilon}}{\sqrt{c_{1}^{\prime \prime} c_{2, j}^{\prime \prime}} \sqrt{\prod_{i=1}^{l} p_{i}}} \prod_{k_{i}^{\prime}>1} p_{i} \prod_{k_{i}^{\prime}=1} \sqrt{p_{i}} \frac{G\left(d s_{1}^{2}\right)^{-\frac{3}{4}} T_{1}^{\frac{1}{4}} \sqrt{T_{2}}}{\sqrt{c} K \sqrt{\beta}} \\
& \times\left(\frac{K^{4+\frac{\epsilon}{100}} c^{2} c_{1}^{\prime \prime 2} c_{2, j}^{\prime \prime 2} d s_{1}^{2}}{T_{1}^{3} T_{2}}\right)^{\frac{3}{4}} \\
& \ll \frac{2^{\omega\left(c_{1}\right)} G K^{2+\epsilon} c c_{1}^{\frac{3}{2}} c_{2}^{\frac{5}{2}+\epsilon} c_{1} c_{2}}{T_{1}^{2} \sqrt{T_{2}}} .
\end{aligned}
$$

Recall that

$$
\omega\left(c_{1}\right) \ll \frac{\log c_{1}}{\log \log c_{1}} .
$$

Thus, fix any $\epsilon>0$ for $c_{1}$ large enough (the case when $c_{1}$ small is trivial),

$$
\omega\left(c_{1}\right) \ll c_{1}^{\epsilon}
$$


Hence, (4.3.1)

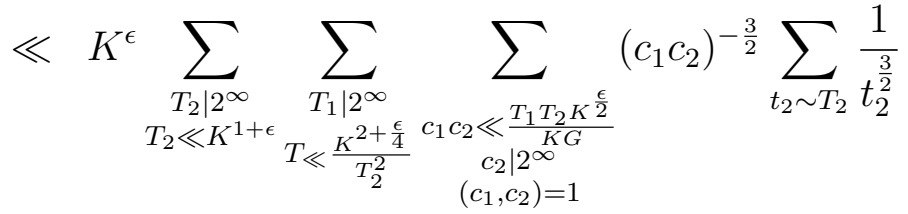

$$
\begin{aligned}
& \times \frac{G K^{2+\epsilon} c_{1}^{\frac{7}{2}} c_{2}^{\frac{9}{2}+\epsilon}}{T_{1}^{2} \sqrt{T_{2}}} \\
& \ll G K^{2+3 \epsilon} \sum_{\substack{T_{2} \mid 2^{\infty} \\
T_{2} \ll K^{1+\epsilon} \epsilon}} \sum_{\substack{T_{1} \mid 2_{2}^{\infty} \\
T_{2} \frac{K^{+}}{T_{2}^{2}}}} \frac{1}{T_{1}^{2} T_{2}}\left(\frac{T_{1} T_{2} K^{\frac{\epsilon}{2}}}{K G}\right)^{3} \\
& \ll G K^{2+5 \epsilon} \sum_{\substack{T_{2} \mid 2^{\infty} \\
T_{2} \ll K^{1+\epsilon}}} \sum_{\substack{T_{1} \mid 2^{\infty} \\
T_{1} \ll \frac{K^{2+\epsilon}}{T_{2}^{2}}}} \frac{T_{1} T_{2}^{2}}{K^{3} G^{3}} \ll \frac{G K^{5 \epsilon} K}{G^{3}} \\
& \leq G K^{5 \epsilon}
\end{aligned}
$$

whenever $G \geq K^{\frac{1}{3}}$. This concludes the proof of our main Theorem. 


\section{BIBLIOGRAPHY}

[1] J. Cogdell and P. Michel, On the complex moments of symmetric power Lfunctions at s=1, Int. Math. Res. Not., 31, (2004), 1561-1617.

[2] J.M. Deshouillers and H. Iwaniec, Kloosterman sums and Fourier cofficients of cusp forms, Invent. Math., 70, (1982), 219-288

[3] W. Duke, J.B. Frielander and H. Iwaniec, Bounds for Automorphic L-functions II, Invent. Math., 115, (1994), 219-239

[4] A. Erdelyi, W. Magnus, F. Oberhettinger and F. Tricomi, Higher Transcendental Functions, New York: McGraw-Hill, 2, 1953.

[5] G. Harcos, Uniform approximate functional equation for principal $L$-functions, IMRN, 18, (2002), 923-932

[6] M. N. Huxley, Area, Lattice, Points, and Exponential Sums,London Mathematical Society Monographs, New series vol. 13, Oxford: Oxford Science Publications, Clarendon Press,1996.

[7] H. Iwaniec, Mean values for Fourier coefficients of cusp forms and sums of Kloosterman sums, Journées Arithmetiqués de Exeter, 56, (1980), 306-321.

[8] H. Iwaniec, Fourier coefficients of modular forms of half-integral weight, Invent. Math., 87, (1987), 385-401

[9] H. Iwaniec, Topic in classical automorphic forms, Graduate Studies of Mathematics, 17, Amer. Math. Soc., Providence, RI., 1997

[10] H. Iwaniec, Spectral Methods of Automorphic Forms, Graduate Studies in Mathematics., , Amer. Math. Soc., Providence, RI, 2002

[11] H. Iwaniec and P. Michel, The second moment of the symmetric square Lfunctions, Ann. Acad. Sci. Fenn. Math., 2, (2001), 465-482.

[12] H. Iwaniec and E. Kowalski, Analytic Number Theory, American Mathematics Society Colloquium Publications, , Amer. Math. Soc., Providence, RI., 2004 
[13] H. Iwaniec, W. Luo and P. Sarnak, Low lying zeros of families of L-functions, I.H.E.S. Publ. Math., 91, (2000), 55-131.

[14] M. Jutila, On spectral large sieve inequalities, Functiones et Approximatio, 28, (2000), 7-18.

[15] M. Jutila and Y. Motohashi, Uniform bound for Hecke L-functions, Acta. Math. , 195, (2005), 61-115.

[16] R. Khan, Non-vanishing of the symmetric square L-function at the central point, Proc. Lond. Math. Soc., 3, 100, (2010), 736-762

[17] J.W.C. Lam, A local large sieve inequality for cusp forms, 26, Fascicule 3, Journal de Théorie des numbers de Bordeaux, (2014), 757-788

[18] J.W.C. Lam, Second moment of the central values of the symmetric square L-functions, Ramanujan Journal, published online on August 12014 ,

[19] N. N. Lebedev, Special functions and their applications, Dover Books on Mathematics, New York, 1972

[20] X. Li, Bounds for $G L(3) \times G L(2) L$-functions and $G L(3) L$-functions, Ann. of Math., 173, 2011, no. 1, 301-336.

[21] W. Luo, Spectral means-values of automorphic L-functions at special points, Analytic Number Theory, Proc. of a Conference in honor of Heini Halberstam, 70, (1982), 219-288.

[22] W. Luo, On shifted convolution of half-integral weight cusp forms, Science China, 53, 9, (2010), 2411-2416.

[23] W. Luo, Central values of the symmetric square L-functions., Proc. Amer. Math. Soc., 140, (2012), no.10, 3313-3322.

[24] W. Luo and P. Sarnak, Mass equidistribution for Hecke eigenforms, Comm. Pure Appl. Math., 56, (2003), 874-891.

[25] W. Luo, and P. Sarnak, Quantum variance for Hecke eigenforms, Ann. Scient. Ec. Norm. Sup., 4 serie, 37, (2004), 769-799

[26] T. Meurman, On exponential sums involving the Fourier coefficients of Maass wave forms, J Reine Angew Math, 384, (1988), 192-207

[27] Y. Motohashi, Spectral Theory of the Riemann Zeta-Function, Cambridge Tracts in Mathematics, Cambridge University Press, Cambridge,127, 2008

[28] H. E. Richert, Lectures on Sieve Methods, Tata Institute of Fundamental Research, Bombay, India, 1976 
[29] G. Shimura, On Modular Forms of Half Integral Weight, Annals of Mathematics , Second Series, 97, No. 3, (1973), 440-481

[30] G. N. Watson, A treatise on the theory of Bessel functions, Cambridge University Press, Cambridge, 127, 1997

[31] Q. Zhang, A local large sieve inequality for the Maass cusp form, preprint. 